University of Louisville

ThinkIR: The University of Louisville's Institutional Repository

Electronic Theses and Dissertations

$12-2014$

\title{
A non-invasive image based system for early diagnosis of prostate cancer.
}

Ahmad Abdusalam Firjani Firjani Naef

University of Louisville

Follow this and additional works at: https://ir.library.louisville.edu/etd

Part of the Computer Engineering Commons

\section{Recommended Citation}

Firjani Naef, Ahmad Abdusalam Firjani, "A non-invasive image based system for early diagnosis of prostate cancer." (2014). Electronic Theses and Dissertations. Paper 1710.

https://doi.org/10.18297/etd/1710

This Doctoral Dissertation is brought to you for free and open access by ThinkIR: The University of Louisville's Institutional Repository. It has been accepted for inclusion in Electronic Theses and Dissertations by an authorized administrator of ThinkIR: The University of Louisville's Institutional Repository. This title appears here courtesy of the author, who has retained all other copyrights. For more information, please contact thinkir@louisville.edu. 


\title{
A NON-INVASIVE IMAGE BASED SYSTEM FOR EARLY DIAGNOSIS OF PROSTATE CANCER
}

\author{
By \\ Ahmad Abdusalam Firjani Naef \\ M.Sc., University of Putra Malaysia, 2002 \\ A Dissertation \\ Submitted to the Faculty of the \\ J. B. Speed School of the University of Louisville \\ in Partial Fulfillment of the Requirements \\ for the Degree of \\ Doctor of Philosophy \\ Department of Computer Engineering and Computer Science \\ University of Louisville \\ Louisville, Kentucky
}

December, 2014 



\title{
A NON-INVASIVE IMAGE BASED SYSTEM FOR EARLY DIAGNOSIS OF PROSTATE CANCER
}

\author{
By \\ Ahmad Abdusalam Firjani Naef \\ M.Sc., University of Putra Malaysia, 2002
}

A Dissertation Approved on

December 4th, 2014

by the Following Reading and Examination Committee:

Adel S. Elmaghraby, Ph.D., Co-Advisor

Ayman El-Baz, Ph.D., Co-Advisor

Olfa Nasraoui, Ph.D.

Ibrahim Imam, Ph.D.

Garth Beache, M.D. 


\section{DEDICATION}

This dissertation is dedicated to my mother, my father, and my wife for their love, patience, and support during the completion of this endeavor. 


\section{ACKNOWLEDGEMENTS}

In the Name of Allah, the Most Gracious, the Most Merciful. All the praises and thanks be to Allah Almighty, the Giver of bountiful blessings and gifts. I would like to thank all those people that contributed to the completion of this thesis.

I would like to express my sincere gratitude to Dr. Adel Elmaghraby and Dr. Ayman El-Baz, my dissertation advisors, for the immeasurable amount of support and guidance they had provided throughout this work. I would like to express my deepest gratitude to Dr. Olfa Nasraoui, Dr.Ibrahim Imam, and Dr.Garth Beache for being on my dissertation committee. And, I want to thank all the people who are part of the research group in the Biolmaging Lab. who have become not only colleagues but also good friends.

Additionally, I would also like to thanks all members of the research group in the Innovative and Emerging Technologies Lab. Special thanks to Dr. Yehia H. Khalil, Omar Abdelwahab. I would like to extend my warmest thanks to the Computer Engineering and Computer Science department staff for their great help and support.

I owe my loving thanks for my wife Eman Alarnaoti without her encouragement and understanding it would have been impossible for me to finish this work. My special gratitude is due to my mother, my Father Abdusallam Firjani Naef, my sisters, and my brothers for their loving support. 


\title{
ABSTRACT \\ A NON-INVASIVE IMAGE BASED SYSTEM FOR EARLY DIAGNOSIS OF PROSTATE CANCER
}

\author{
Ahmad Abdusalam Firjani Naef
}

December 04, 2014

Prostate cancer is the second most fatal cancer experienced by American males [1]. The average American male has a $16.15 \%$ chance of developing prostate cancer, which is $8.38 \%$ higher than lung cancer, the second most likely cancer. The current in-vitro techniques that are based on analyzing a patients blood and urine have several limitations concerning their accuracy. In addition, the prostate Specific Antigen (PSA) blood-based test, has a high chance of false positive diagnosis, ranging from $28 \%-58 \%$. Yet, biopsy remains the gold standard for the assessment of prostate cancer, but only as the last resort because of its invasive nature, high cost, and potential morbidity rates. The major limitation of the relatively small needle biopsy samples is the higher possibility of producing false positive diagnosis. Moreover, the visual inspection system (e.g., Gleason grading system) is not quantitative technique and different observers may classify a sample differently, leading to discrepancies in the diagnosis. As reported in the literature that the early detection of prostate cancer is a crucial step for decreasing prostate cancer related deaths. Thus, there is an urgent need for developing objective, non-invasive image based technology for early detection of prostate cancer. 
The objective of this dissertation is to develop a computer vision methodology, later translated into a clinically usable software tool, which can improve sensitivity and specificity of early prostate cancer diagnosis based on the well-known hypothesis that malignant tumors are will connected with the blood vessels than the benign tumors. Therefore, using either Diffusion Weighted Magnetic Resonance imaging (DW-MRI) or Dynamic Contrast Enhanced Magnetic Resonance Imaging (DCE-MRI), we will be able to interrelate the amount of blood in the detected prostate tumors by estimating either the Apparent Diffusion Coefficient (ADC) in the prostate with the malignancy of the prostate tumor or perfusion parameters. We intend to validate this hypothesis by demonstrating that automatic segmentation of the prostate from either DW-MRI or DCE-MRI after handling its local motion, provides discriminatory features for early prostate cancer diagnosis.

The proposed CAD system consists of three majors components, the first two of which constitute new research contributions to a challenging computer vision problem. The three main components are: (1) A novel Shape-based segmentation approach to segment the prostate from either low contrast DW-MRI or DCE-MRI data; (2) A novel iso-contours-based non-rigid registration approach to ensure that we have voxel-on-voxel matches of all data which may be more difficult due to gross patient motion, transmitted respiratory effects, and intrinsic and transmitted pulsatile effects; and (3) Probabilistic models for the estimated diffusion and perfusion features for both malignant and benign tumors. Our results showed a 98\% classification accuracy using Leave-One-Subject-Out (LOSO) approach based on the estimated ADC for 30 patients (12 patients diagnosed as malignant; 18 diagnosed as benign). These results show the promise of the proposed image-based diagnostic technique as a supplement to current technologies for diagnosing prostate cancer. 


\section{TABLE OF CONTENTS}

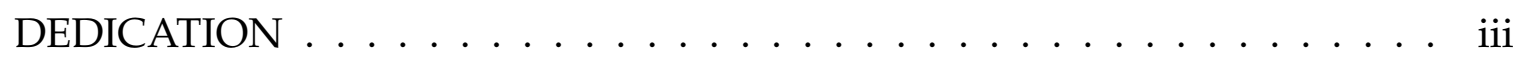

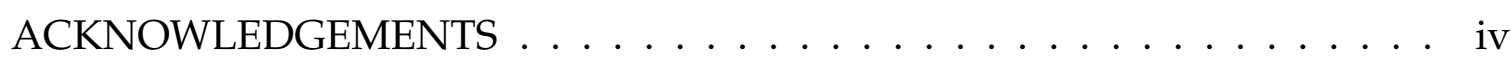

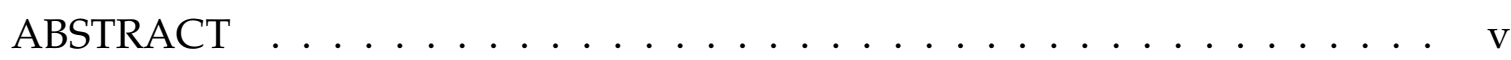
LIST OF TABLES $\ldots \ldots \ldots \ldots \ldots \ldots \ldots \ldots \ldots \ldots \ldots \ldots \ldots \ldots \ldots$ LIST OF FIGURES $\ldots \ldots \ldots \ldots \ldots \ldots \ldots \ldots \ldots \ldots \ldots \ldots$ xi LIST OF ALGORITHMS $\ldots \ldots \ldots \ldots \ldots \ldots \ldots \ldots$. . . . . . . . . . . . CHAPTER

I. PROSTATE ANATOMY AND CURRENT DIAGNOSTIC TECHNIQUES 1

A. PROSTATE ANATOMY $\ldots \ldots \ldots \ldots \ldots \ldots$

B. CURRENT DIAGNOSTIC TECHNIQUES . . . . . . . . . . . 3

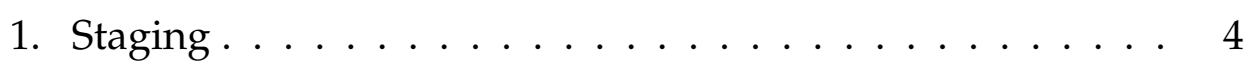

2. Treatment ................ 6

3. Diagnostic Techniques . . . . . . . . . . . . . 6

C. Limitation of the Current Diagnostic Techniques . . . . . . . . 9

D. THE NEED FOR THIS WORK $\ldots \ldots \ldots$

1. Image Segmentation Methods . . . . . . . . . . . . 12

2. Image Registration Methods . . . . . . . . . . . . . . 13

E. DISSERTATION ORGANIZATION . . . . . . . . . . . . 14

II. IMAGING THE PROSTATE IN CANCER $\ldots \ldots \ldots \ldots$ 
A. MAGNETIC RESONANCE IMAGING $\ldots \ldots \ldots$

1. Structural MRI . . . . . . . . . . . . . . . 20

2. Dynamic Contrast-Enhanced MRI (DCE-MRI) . . . . . . . . 20

3. Magnetic Resonance Spectroscopy Imaging (MRSI) . . . . . 22

4. Diffusion-Weighted Imaging (DWI) . . . . . . . . . . . 22

B. QUANTIFICATION OF PROSTATE CANCER USING MR IMAGES . . . . . . . . . . . . . . . . . . 23

a. Early Detection of Prostate Cancer using (DCE-MRI) . . 23

b. Diffusion-Weighted MRI . . . . . . . . . . 25

III. IMAGE BASED RELATED WORK FOR EARLY DIAGNOSES OF PROSTATE CANCER . . . . . . . . . . . . . . . . . . 28

IV. A NOVEL IMAGE-BASED APPROACH FOR EARLY DETECTION OF PROSTATE CANCER USING DCE-MRI . . . . . . . . . . . . . . 44

A. INTRODUCTION . . . . . . . . . . . . . . . 45

B. Methods . . . . . . . . . . . . . . . . . 4 47

1. Segmentation of the Prostate Using a Joint MGRF Model . . 47

a. Conditional Intensity Model . . . . . . . . . . . . 51

b. Spatial Voxel Interaction Model . . . . . . . . . . . 52

c. Probabilistic Shape Model . . . . . . . . . . . . . . . . . 54

2. Nonrigid Registration . . . . . . . . . . . . . 59

3. Classification, Performance Analysis, and Visualization of Perfusion Indexes . . . . . . . . . . . . . 62

a. Color Map Generation and Tumor Boundary Determination .................... 62

C. PERFORMANCE EVALUATION AND VALIDATION . . . . . . 64

D. PATIENT DATA AND DCE-MRI ACQUISITION PROTOCOL . 66 
1. Prostate Segmentation Results . . . . . . . . . . . 67

2. Diagnostic Results . . . . . . . . . . . . . . . . 72

E. SUMMARY ......................... 79

V. A NOVEL IMAGE-BASED APPROACH FOR EARLY DETECTION OF PROSTATE CANCER USING DIFFUSION-WEIGHTED MRI . . 80

A. INTRODUCTION . . . . . . . . . . . . . . . . 81

B. MATERIALS AND METHODS . . . . . . . . . . . . . . . 81

1. Delineation of the Prostate Borders . . . . . . . . . . 82

2. Nonrigid Registration ... . . . . . . . . . . . 84

3. Diffusion Characterization and Tumor Boundary Determi-

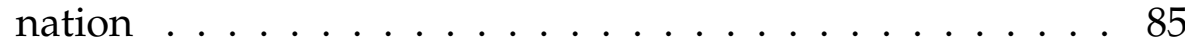

C. EXPERIMENTAL RESULTS . . . . . . . . . . . . . . . 89

1. Patients and Data Acquisition ... . . . . . . . 89

2. Segmentation Results . . . . . . . . . . . . . 90

3. Diagnostic Results . . . . . . . . . . . . . . . . . 91

D. SUMMARY ........................... 96

VI. CONCLUSIONS AND FUTURE WORK . . . . . . . . . . . . . . 97

REFERENCES . . . . . . . . . . . . . . . . . . . 100

APPENDIX

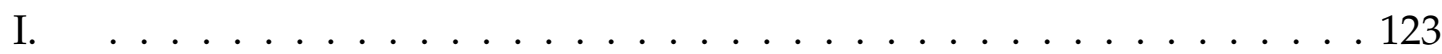

A. LIST OF ABBREVIATIONS . . . . . . . . . . . . . . . . . 123

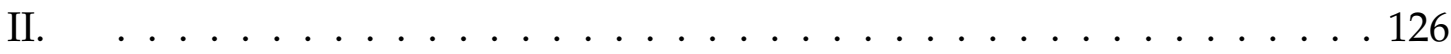

A. NOMENCLATURE . . . . . . . . . . . . . . . . 126

CURRICULUM VITAE . . . . . . . . . . . . . . . . . . . . . . . . 130 


\section{LIST OF TABLES}

TABLE.

PAGE

1. Outlines normal age-specific PSA . . . . . . . . . . . . . . . 9

2. Summary of the discussed prostate segmentation and registration techniques and their experimental performance. Note that DIM and AL stand for data dimension (i.e., 2D, 3D, or 4D) and the automation level (i.e., automated or semi-automated), respectively. . . . . . . . . . . . . 35

3. Summary of MRI-based CAD systems for prostate cancer detection and diagnosis, including segmentation method, selected features, classifier, and experimental performance. . . . . . . . . . . . . 38

4. Error statistics of the proposed segmentation approach. Note that SD, stands for standard deviation.

5. Comparative segmentation accuracies of the proposed prostate segmentation against the shape-based (SB) approach [2] in comparison to the experts ground truth (SD standard deviation). . . . . . . . . . . . . 69

6. Error Statistics over all test data sets. . . . . . . . . . . . . . . . 90

7. A Comparative segmentation accuracy over all test data sets for our approach and [2]. Note that STD stands for standard deviation. . . . . . . . 92

8. Area under the ROC curve for training subjects, testing subjects, and combined (training and testing subjects) . . . . . . . . . . . . 92 


\section{LIST OF FIGURES}

FIGURE.

1. Schematic illustration of an anatomical view of the lower abdomen area that contains the prostate (Courtesy of [3]) . . . . . . . . . . . . . 2

2. Schematic illustration of the front and side cross-sectional views of the prostate showing different prostate zones (Courtesy of [4]). . . . . . . . . 3

3. Illustration of Malignant Tumor (Courtesy of [5]) . . . . . . . . . . . . . 4

4. The stages of prostate cancer (Courtesy of [6]) . . . . . . . . . . . . 5

5. Diagnosing scheme for prostate cancer. . . . . . . . . . . . . . . . 9

6. An illustration of Transperineal and transrectal approaches to prostate biopsy. A thin needle is inserted through the rectum or through the perineum parallel to the urethra to perform the operation, and the procedure is usually directed by an imaging device (e.g., ultrasound) to help in placing the needle into the right location (Courtesy of [7]) . . . . . . . 10

7. Schematic illustration of different medical image modalities for imaging the prostate in cancer. . . . . . . . . . . . . . . . . . . .

8. Different types of prostate medical images: (a) ultrasound image (Courtesy of [8]), (b) computed tomography (CT) image (Courtesy of [7]), and (c) $T_{2}$-weighted (MRI) image. . . . . . . . . . . . . . .

9. Different specialized MRI acquisition techniques. . . . . . . . . . . . .

10. MRI Prostate images taken at different time points post the adminstration of the contrast agent showing the change of the contrast as the contrast agent perfuse into the prostate tissues.

11. (a) A typical time-intensity curve of the average intensity of the prostate measured before and after contrast agent adminstration. The curve illustrates typical perfusion-related indexes that can be estimated and used for diagnosis of the prostate cancer. (b) Prostate colored by wash-in and

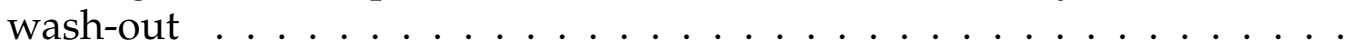


12. Examples of MRS. There is a single choline, polyamine, and creatine peak. 22

13. Diffusion MR images for the prostate at (a) $b$-value of $0 \mathrm{~s} / \mathrm{mm}^{2}$ and (b) $b$-value of $800 \mathrm{~s} / \mathrm{mm}^{2} \ldots \ldots \ldots \ldots \ldots$

14. A block diagram of the proposed CAD system for the early diagnosis of prostate cancer using DCE-MRI. The time series data is first corrected for motion artifacts. Then, the prostatic tissue is segmented and the agent kinetic curves are constructed. Finally, perfusion-related parameters are extracted from the kinetic curves and are used for the classification of both malignant and benign cases. . . . . . . . . . . . . . . . 25

15. Flowchart of the proposed CAD system for automatic detection of cancer from 3D DWI. An automatic prostate segmentation method is applied to isolate the prostate from the surrounding anatomical structures. Following segmentation, a non-rigid registration approach is employed to account for any local deformation that could occur in the prostate during the scanning process. Three features are extracted from registered diffusion data. The features are summarized by a supervised classifier to calculate the likelihood of malignancy. . . . . . . . . . . . . . . . 26

16. Pixel-wise parametric map display of the diffusion information and the spatial interactions between the prostate voxels. The red and blue hues of each color scale correspond to enhanced and reduced perfusion, respectively. . . . . . . . . . . . . . . . . . 27

17. The proposed CAD system for early detection of prostate cancer . . . . . 48

18. Illustration of the Joint Markov-Gibbs random field (MGRF) image model of the prostate DCE-MRI. . . . . . . . . . . . . . . . . . . 50

19. 3D $2^{\text {nd }}$ order MGRF neighborhood system. Note that the reference voxel is shown in red and $d$ represents the absolute distance between two voxels in the same and adjacent MRI slices, or cross-sections.

20. Forming the prostate shape prior onto 2D: $(a)$ training samples, $(b)$ manually segmented prostate regions, and $(b)$ their affine MI-based alignment. Note that the registration enhances the overlap between the prostate objects and thus reduce the variability of the final estimated shape. . . . .

21. 3D prostate shape model projected onto $2 \mathrm{D}$ axial (a), saggital (b), and coronal (c) planes for visualization. 
22. Gray-coded (a) and color-coded (b) axial view of the prostate shape prior. initial region map. The final Bayesian segmentation is performed using the identified joint MGRF model of the DCE-MRI data and its region maps. In total, the proposed prostate segmentation approach involves the steps summarized in Algorithm IV.B.1.c . . . . . . . . . . . . . 56

23. Illustration of the iso-contours generation: the reference (a) and target (d) images, the reference (b) and target (e) distance maps, and reference

(c) and target (f) iso-contours.

24. 2-D schematic illustration of co-allocation of the point-to-point correspondences between two boundaries by a potential field.

25. Estimating $\delta$ from the perfusion curve as the difference between the peak and initial signal of image sequences. . . . . . . . . . . . . . . .

26. Enhanced perfusion estimation and continuity analysis using the $3 \mathrm{D}$ GGMRF image model. . . . . . . . . . . . . . . . . . . . .

27. 2-D schematic illustration of measuring segmentation errors between the ground truth $\mathbf{G}$ and automatic segmentation $\mathbf{C}$ obtained by the our

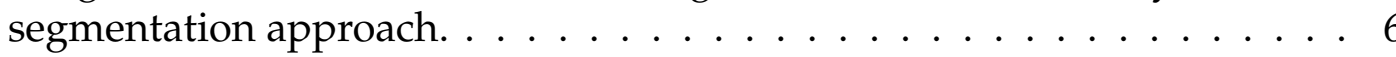

28. Segmentation results: the segmentation results with error referenced to the ground truth $G$ is outlined in yellow (False Negative (FN): pixels segmented as the prostate in $G$ but not segmented as the prostate with our approach) and red (False Positive (FP): pixels segmented as the prostate with our approach but not segmented as the prostate in the $G$. . . . .

29. Segmentation results of the first subject after 30 iteration: (a) typical prostate DCE-MRI images. (b) the ground truth (c) segmentation results by intensity, shape prior, and spatial interaction $(\mathrm{d})$ segmentation results by intensity, and spatial interaction only. . . . . . . . . . . . .

30. Accuracy of our segmentation in comparison with [2]. Our segmentation is outlined in blue and [2] in red with reference to the ground truth $\mathrm{G}$ in white.

31. Estimation of the perfusion parameters for the classification of prostate tumor (a) Dynamic sequence showing prostate-averaged voxel intensity versus time, along with images at three different time (b) Prostate colored by wash-in and wash-out.

32. Selected normalized signal intensity, averaged over the entire prostate, with respect to the timing of contrast agent delivery for malignant (red) and benign (blue) subject. . . . . . . . . . . . . . . . . . . . 73 
33. Color-coded maps for four of the test subjects before and after the 3-D GGMRF smoothing with $\rho=1, \lambda=5, \beta=1.01, \alpha=2$, and $\eta_{s, r}=\sqrt{2}$ and their respective color-coded maps. The red and blue ends of the color scale relate to the maximum and minimum changes, respectively.

34. Color-coded maps of local tumor progression overlaid on anatomic DCEMRI data for one benign subject. The determined tumor contours are shown in blue.

35. Color-coded maps of local tumor progression overlaid on anatomic DCEMRI data for one benign subject. The determined tumor contours are shown in blue.

36. Dynamic contrast-enhanced MRI images of the pelvis with local tumor progression of malignant subject. The determined tumor contours are shown in blue.

37. Dynamic contrast-enhanced MRI images of the pelvis with local tumor progression of another malignant subject. The determined tumor contours are shown in blue. . . . . . . . . . . . . . . . 78

38. Flowchart of the proposed CAD system for automatic detection of cancer from 3D DWI.

39. 3D $2^{\text {nd }}$ order MGRF neighborhood system. Note that the reference voxel is shown in red and $a$ represents the absolute distance between two voxels in the same and adjacent MRI slices, or cross-sections . . . . . . . . . .

40. Illustration of the iso-contours generation: the reference and target distance maps $(a, b)$, and their iso-contours $(c, d) \ldots \ldots \ldots$

41. Enhanced perfusion estimation and continuity analysis using the $3 \mathrm{D}$ GGMRF image model. . . . . . . . . . . . . . . .

42. Color-coded maps for three of the test subjects (column wise) before and after the 3-D GGMRF smoothing. The red and blue ends of the color scale relate to the maximum and minimum changes, respectively. . . . .

43. Pixel-wise parametric map display of the diffusion information and the spatial interactions between the prostate voxels. The red and blue hues of each color scale correspond to enhanced and reduced perfusion, re-

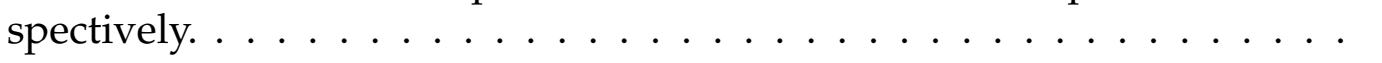

44. Segmentation result and their associated FN (green) and FP (red) errors referenced to the ground truth $G$. 
45. 3-D prostate segmentation projected onto 2-D. (a) 2-D visualization for our segmented prostates for three of the test subjects, (b) our segmentation (red) in comparison with the ground truth (white), and (c) the segmentation with the algorithm in [2] (red) comparison with the ground truth. . . . . . . . . . . . . . . . . . . 93

46. Receiver operating characteristic curves for training subjects, testing subjects, and combined (training and testing subjects). CAD performance is with respect to classification produced by the expert. . . . . . . . . . . . 94

47. Diffusion MR images for the prostate at (a) b-value of $0 \mathrm{~s} / \mathrm{mm}^{2}$, (b) b value of $800 \mathrm{~s} / \mathrm{mm}^{2}$, and (c) tumor progression for four of the test subjects. 95 


\section{LIST OF ALGORITHMS}

ALGORITHM.

PAGE

1. 3D Prostate Shape Model . . . . . . . . . . . . . . . . . . 57

2. Prostate Segmentation Approach . . . . . . . . . . . . . . 58

3. Laplace-Based Iso-Contours Nonrigid Registration . . . . . . . . . 61

4. Key Steps for prostate Segmentation . . . . . . . . . . . . . . 85 


\section{CHAPTER I}

\section{PROSTATE ANATOMY AND CURRENT DIAGNOSTIC TECHNIQUES}

Prostate cancer is the most common malignancy among men. Despite its prevalence, Prostate cancer is a curable condition in many cases. In America for example, the five-year survival rate of prostate cancer for men is $100 \%$ among patients diagnosed with localized or regional disease and only $31 \%$ among men di-

agnosed at a distant stage [9]. Mortality often happens when patients are affected by metastasis of the cancer to the bones. At that stage, surgical and hormonal treatments are not very effective. Therefore, the early diagnosis and staging of the disease play an important role in the choice and the success of the treatment.This chapter provides the background to adequately understand the anatomy of the prostate, prostate cancer, staging of the prostate cancer, and screening modalities.

\section{A. PROSTATE ANATOMY}

The term prostate means "to stand in front of ", which is derived from the Greek prohistani. This expression was adopted by Herophilus of Alexandria in 335 B.C. to describe the organ located in front of the urinary bladder [3]. The prostate gland location is illustrated in Figure 1. It measures approximately $4 \times 3 \times 2 \mathrm{~cm}$. The anterior surface lies at the back of the retropubic space and is connected to the pubic bone by the puboprostatic ligaments [10].

The prostate is divided into three main zones: the central zone $(\mathrm{CZ})$, the 


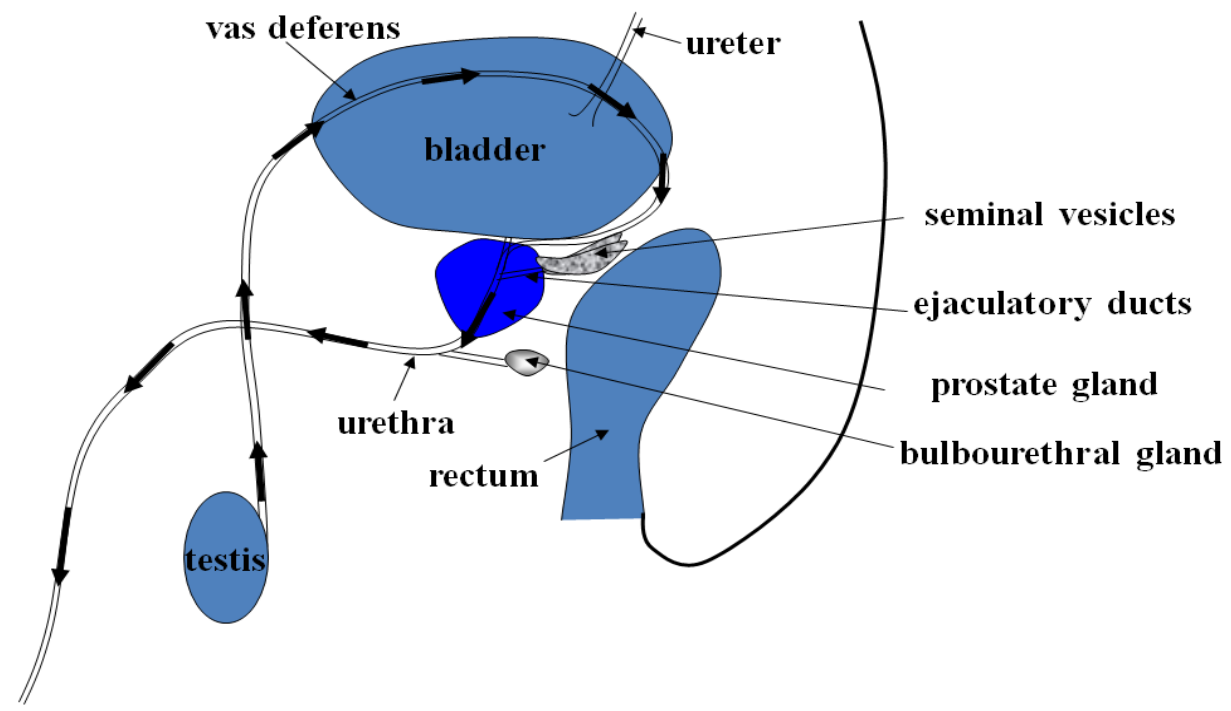

FIGURE 1: Schematic illustration of an anatomical view of the lower abdomen area that contains the prostate (Courtesy of [3]).

peripheral zone (PZ), and the transition zone (TZ), excluding the urethra and periurethral glands (Figure 2). The peripheral zone represents approximately $65 \%$ of the prostatic volume. The second largest part of the prostate is the central zone. It is represents approximately $25 \%$ of the prostatic volume. The third zone of the prostate is called the transition zone, which represents only $5 \%$ to $10 \%$ of a typical prostatic volume. The prostate gland is divided into three zones according to their function:

- The transition zone (TZ) consists of two independent pear shaped lobes situated laterally on either side of the prostatic urethra together with periurethral glands. This is the zone where benign prostatic hypertrophy $(\mathrm{BPH})$ occurs.

- The central zone (CZ) surrounds the transitional zone posteriorly and encloses the ejaculatory ducts. It is the zone most commonly affected by inflammatory processes (e.g. prostatitis). In benign prostatic hyperplasia, the transitional zone and periurethral glands enlarge considerably compressing the central zone to form a thin layer, the so called surgical pseudocapsule. 
- The peripheral zone (PZ) is the most posterolateral glandular component of the prostate. The ratio of peripheral zone to central gland tissue gradually decreases from the apex to the base of the gland. The peripheral zone can be compressed and distorted by $\mathrm{BPH}$ and it is the site of the majority of prostate cancers [11].

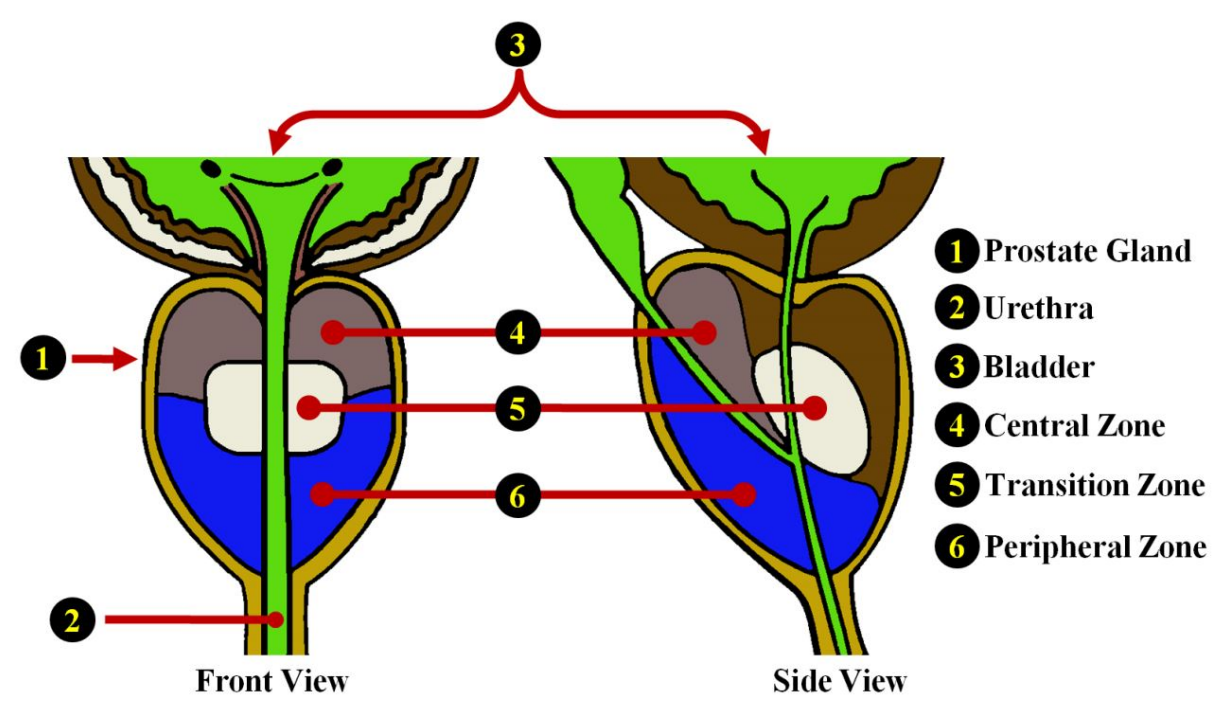

FIGURE 2: Schematic illustration of the front and side cross-sectional views of the prostate showing different prostate zones (Courtesy of [4]).

\section{B. CURRENT DIAGNOSTIC TECHNIQUES}

Inside the prostate, groups of cells may form benign or malignant tumors (see Figure 3). The cancerous cells may form within the prostate but grow too slowly to cause problems. In other cases, cancerous tumors may grow inside the prostate, then spread [6]. The prostate cancerous cells are characterized as follows:

- Noncancerous cells: These may grow inside the prostate as a man ages. This condition is called benign prostatic hyperplasia (BPH). These growths often 


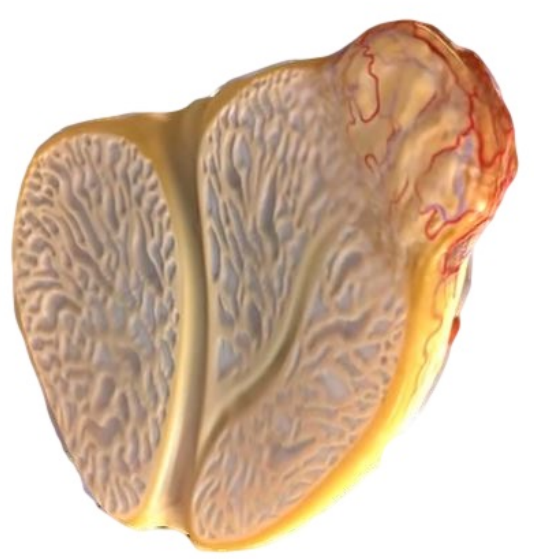

FIGURE 3: Illustration of Malignant Tumor (Courtesy of [5]).

squeeze the urethra, causing symptoms such as difficulty urinating.

- Pre-cancerous cells: These cells do not appear normal, but they do not present all the characteristics of cancerous cells. They can not be felt during a physical exam and they do not produce symptoms.

- Cancerous cells: These form most often in the prostate's outer tissue. Cancer cells may stay inside the prostate or they may spread to nearby organs and tissues, such as the bladder and seminal vesicles (local spread) or to the lymph nodes near the prostate (regional spread). Cancer cells can also spread through the bloodstream to more distant structures such as the bones (distant metastasis). Many early-stage tumors do not squeeze the urethra, so they may not cause symptoms. In some cases, tumors can be felt during an exam.

1. Staging

Staging is used to classify how far the cancer has spread. Cancer is assigned to one of four stages as, shown in figure 4: 
Stage I

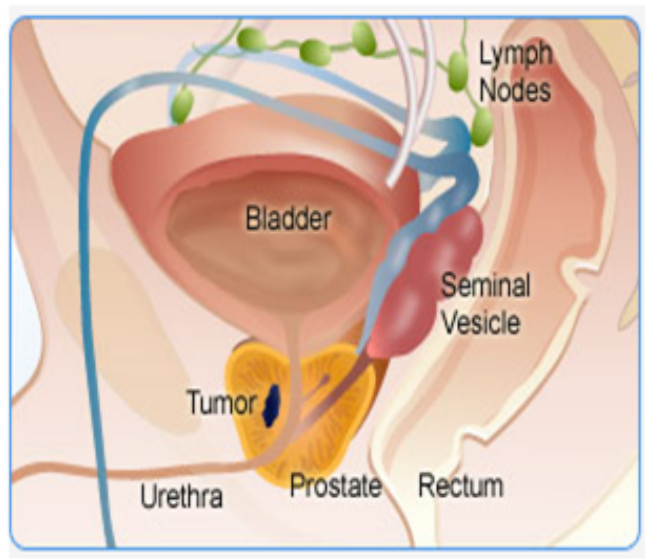

Stage III

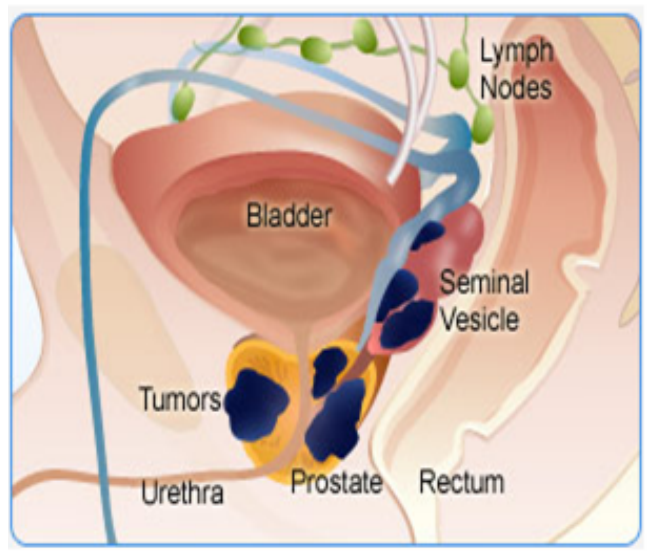

Stage II

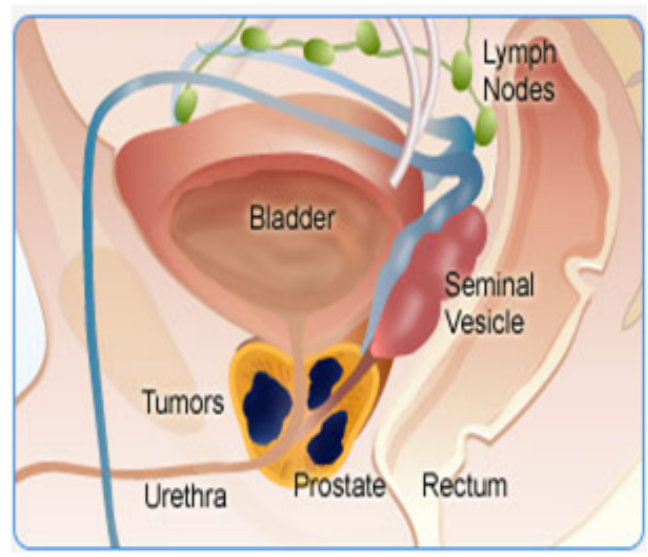

Stage IV

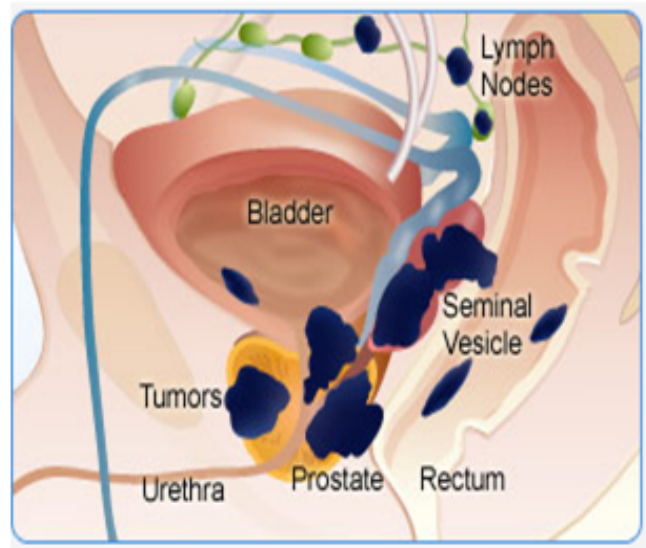

FIGURE 4: The stages of prostate cancer (Courtesy of [6]).

- Stage I: Early cancer that is confined to a microscopic area and is too small to feel when palpated.

- Stage II: The doctor can palpate the tumor, but it is confined only to the prostate gland.

- Stage III: The cancer has spread to nearby tissues.

- Stage IV: The cancer has spread to the lymph nodes, bones, lungs or other areas, distant from the original tumor. 
2. Treatment

Four types of standard treatment for prostate cancer have been established [6]:

- Surgery is a first choice if the prostate cancer has not spread outside the gland (stage I or II cancers). In this surgery, the surgeon removes the entire prostate gland. This type of surgery is called a radical prostatectomy.

- Radiation therapy uses high-energy radiation, such as x-rays to kill cancer cells. Radiation is used as the initial treatment for cancers that have grown outside of the prostate gland and into nearby tissues, it is als used to reduce the size of the tumor (stage III cancers).

- Hormone therapy prevents testosterone from reaching prostate cancer cells. This therapy is used if the patient is not able to have surgery or radiation or if the cancer has grown beyond the prostate gland (stage IV).

- Expectant management, or active surveillance, describes the monitoring of the prostate cancer with prostate-specific antigen (PSA) blood tests, digital rectal exams (DREs), and prostate biopsies every three to six months to see changes. Expectant management is used when it is difficult to interpret if the cells are malignant or not. Because, the PSA arises from both benign and malignant epithelial cells, PSA usually increases over time.

3. Diagnostic Techniques

Most men, diagnosed with prostate cancer, have no symptoms and only find their cancer due to screening. However, in advanced cases of prostate cancer, there are symptoms such as bladder outlet obstruction, acute urinary retention, 
neurological symptoms of cord compression or pathologic fractures secondary to bony metastases. The currently used screening tests are as follow:

- Digital Rectal Exam (DRE): The DRE test is carried out by a skilled physician who manually feels for any abnormalities in the prostate gland through the rectum. The accuracy of this examination is not high, averaging 39\%. Nevertheless, DRE is inexpensive, easy to perform, and can detect most of the tumors of a sufficient volume. However, the DRE alone can not be relied on to detect prostate cancer.

- Serum PSA: is considered as the best serum marker in the early detection of prostate cancer. PSA is an enzyme which is secreted by prostatic cells. PSA was first identified and purified in the 1970s. However, its widespread use in clinical urology did not occur until the 1980s. The PSA testing is minimally invasive, simple and safe. Serum PSA elevation may indicate the presence of prostatic disease (including prostate cancer, benign prostatic hypertrophy, urinary retention and prostatitis) or result from prostate manipulation such as transrectal biopsy and prostatic massage. Elevation of PSA above $4 \mathrm{ng} / \mathrm{mL}$ carries a $22 \%$ probability of prostate cancer, and a further increase above $10 \mathrm{ng} / \mathrm{mL}$ raises the cancer risk to $63 \%$ [12-14]. As PSA values rise with age, there is an agreed cut-off level for different age groups. Although this is not universally accepted, as men may harbor prostate cancer despite low levels of serum PSA, the use of an age-specific normal range for PSA values increases the positive predictive value of PSA testing. Table 1 details the normal range of values according to age.

- PSA Density: is defined as the PSA per unit prostate volume. It is useful to differentiate between prostate cancer and benign hyperplasia. The prostate volume can be found from TRUS screening.

- PSA Velocity:is defined as the rate of change of serum PSA with time. 
- TransRectal UltraSound (TRUS) and TRUS Biopsy: TRUS is used to display the prostate, visualize the cancer, and guide the needles to obtain biopsies from the prostate. The most common approaches to biopsies are transrectal and transperineal (see Figure 6). In the transrectal procedure, a 2D US probe is equipped with a needle guide to access to the prostate through the rectal wall [7]. The needle trajectory is aligned with the TRUS image enabling the visualization of the needle trajectory and placement. Since some lesions are isoechoic, thus not visible in US images, it is necessary to sample the prostate in a systematic randomized way. The gland is usually divided into six or more zones of equal volume and one or more core is randomly collected from each zone. In initial biopsies, the needles are usually laterally directed to the peripheral zone, since $70 \%$ of the lesions are situated in this area. These samples are later analyzed in laboratory for evidence of cancerous tissue. Although a large number of biopsies are done with a transrectal approach, as explained above, some urologists prefer to use a transperineal approach. In this case, the image is still acquired by TRUS, but needle insertion is done through the skin between the scrotum and the anus. This procedure is carried out with the patient under local or general anaesthesia [15]. The accuracy of TRUS Biopsy in detecting cancer is in the range of $57 \%$ to $76 \%$.

- Gleason score The samples of tissue from the biopsy are graded based on how it looks under a microscope. A low Gleason score means the cancer tissue is similar to normal prostate tissue and the tumor is less likely to spread; a high Gleason score means the cancer tissue is very different from normal and the tumor is more likely to spread [12].

The main diagnostic tools for prostate cancer are digital rectal exam (DRE) and serum concentration using prostate specific antigen (PSA) blood test. If any of the previous screening modalities suggest any abnormalities, biopsies should be done, 


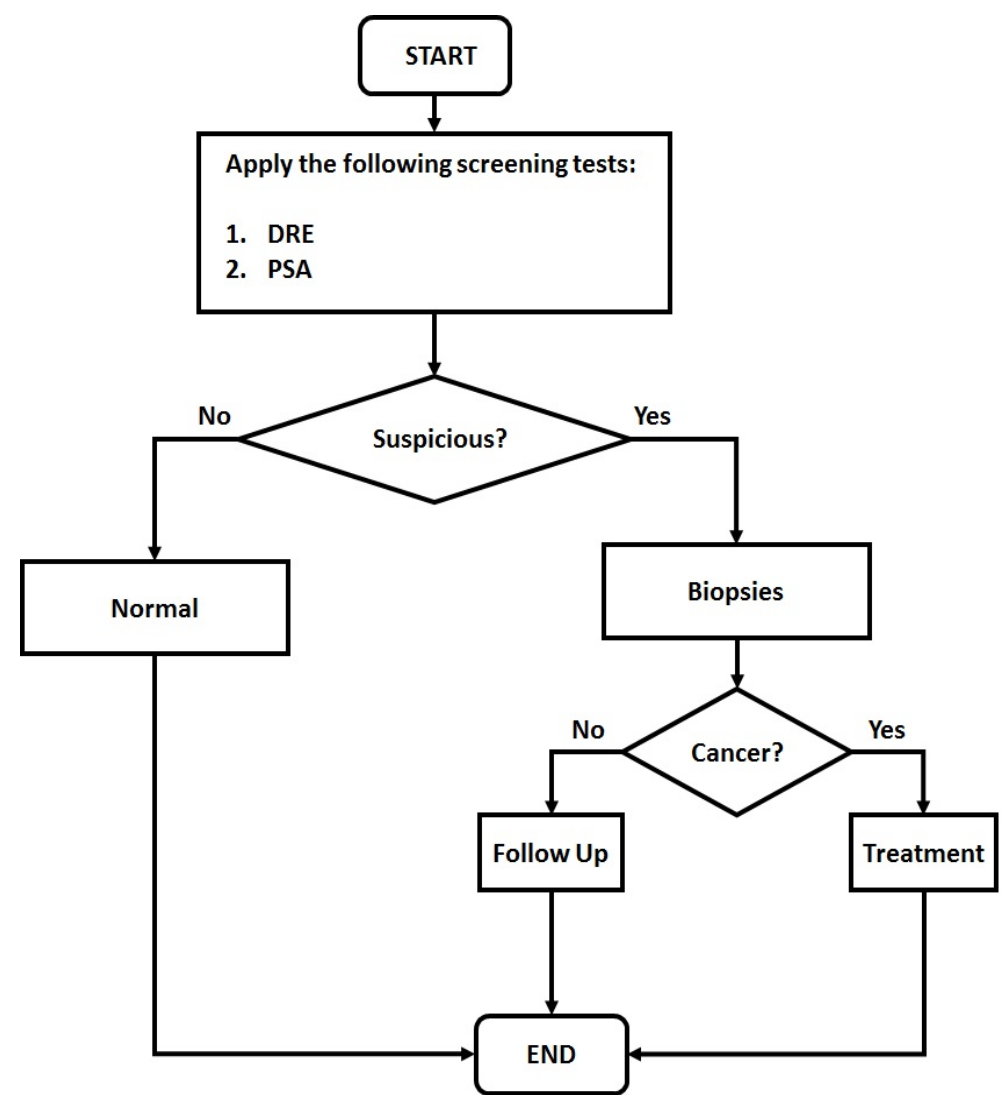

FIGURE 5: Diagnosing scheme for prostate cancer.

TABLE 1: Outlines normal age-specific PSA

\begin{tabular}{cc}
\hline \hline Age group & Normal PSA value \\
\hline$>60$ & PSA $\leq 3$ \\
$60-69$ & PSA $\leq 4$ \\
$>70$ & PSA $\leq 5$ \\
\hline
\end{tabular}

and then examined by a pathologist. The chart in Figure 5 displays the diagnostic scheme for prostate cancer [3].

C. Limitation of the Current Diagnostic Techniques

The currently used techniques for diagnosing prostate cancer are clearly unsatisfactory. For example, prostate specific antigen (PSA) screening does not offer 


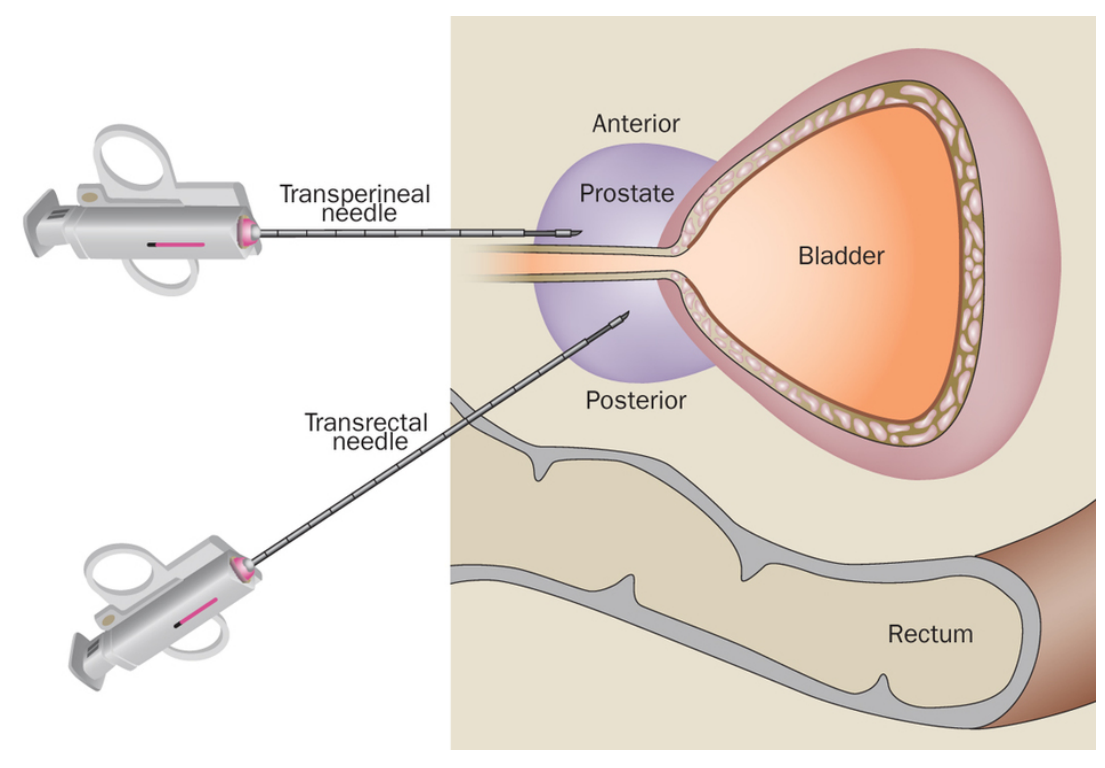

FIGURE 6: An illustration of Transperineal and transrectal approaches to prostate biopsy. A thin needle is inserted through the rectum or through the perineum parallel to the urethra to perform the operation, and the procedure is usually directed by an imaging device (e.g., ultrasound) to help in placing the needle into the right location (Courtesy of [7])

accurate information about the location and extend of the lesion(s). In addition, PSA is associated with a high risk of over diagnosis. TRUS Biopsy is widely used for diagnosis of prostate cancer. However, this technique presents some drawbacks. Because the cores are sampled in a blinded way, without the aid of any visual references (in particular with respect to 3D space), its very difficult to know the correct position of the biopsy inside the gland, since the TRUS images have low signal to noise ratio (SNR) $[7,16]$. Therefore, its impossible to sample the exact same site in a later exam to investigate the development of a small cancer lesion over time. Furthermore, with the current technique, it is possible to miss the cancer since the exam performs a non-exhaustive systematic search for an invisible target. This results in a low detection rate of about 30 to $40 \%$ in traditional $12-$ core biopsies and even with saturation biopsies with 18-cores similar results have been obtained $[15,17]$. Even after an initial extended biopsy, followed by a second, 
third, and fourth saturation biopsy, prostate cancer was still detected in 18\%, 17\%, and $14 \%$ of patients, respectively [18]. Being so, a large number of false negatives are inherent to the process, and a negative result cannot exclude cancer diagnosis. Patients with maintained suspicions may have to repeat biopsy series. Gleason scores require biopsies to invasively collect tissue samples. Therefore, despite all its high costs and morbidity rates, biopsy is still remaining as the golden standard for diagnosis of prostate cancer. However,it is resort.

On the other hand, imaging tests are favorable since they provide reliable information about the size and shape of prostate gland and can localize the cancer foci, which would improve the accuracy of diagnosis and enable more efficient treatment.

\section{THE NEED FOR THIS WORK}

The main objective of this dissertation is to develop a computer-aided diagnostic (CAD) system for early detection of prostate cancer from both (DCE-MRI) and (DW-MRI). Non-invasive approaches for prostate cancer diagnosis have been widely employed, due to their potential to provide superior resolution that plays an important role in, for example, assessing pathological tissues, locations and extent of the tumor, directing biopsies, planning proper therapy, and evaluating therapeutic results. A generic framework for the analysis of prostate cancer images can include one or more of the following: (i) segmentation approaches to extract the prostate, (ii) registration techniques for motion correction, (iii) construction of perfusion curves to distinguish between normal and abnormal tissues, (iv) estimation of perfusion-related parameters, (v) modeling of the extracted parameters, and (vi) classification and diagnosis. Since the main steps of any computer-assisted diagnosis (CAD) system for prostate cancer are image segmentation and image 
registration, below we will briefly overview the popular techniques for image segmentation and registration.

\section{Image Segmentation Methods}

Accurate segmentation of the prostate is fundamental for reliable and robust non-invasive analysis of prostate cancer images. Both accuracy and automation of the segmentation technique are of considerable importance. First, automated segmentation is important because it facilitates the complete automation of the CAD system, minimizes the effect of the operator-dependency, and makes the diagnosis process reproducible. On the other hand, accuracy of segmentation is essential for correct diagnosis because many crucial indexes (e.g., features for discriminating benign and malignant lesions) are based on the contour, shape and texture of the prostate. These features can be effectively extracted after the desired boundary is correctly detected. Popular prostate segmentation techniques can be divided into four major categories: (i) rule-based approaches, (ii) statisticalbased approaches, (iii) atlas-based approaches, and (iv) deformable models-based approaches. A brief overview and the limitations of the existing segmentation approaches are given below.

- Rule-based techniques aim to partition an image into pixels of two or more values through comparison of pixel values. Most popular approaches are thresholding, region growing, and region split-and-merge. However, threshold based segmentation approaches are not accurate in the case of gray levels similarities between the prostate and the surrounding tissues. In addition, region growing-based approaches are sensitive to initialization and often need user assistance to select proper seed points.

- Statistical-based techniques involves parametric or nonparametric probabil- 
ity models of appearance and shape of goal objects, e.g., Bayesian or maximum likelihood inference. The main limitation of current statistical models is that the tails of the prostate distributions usually overlap, so it is difficult if not impossible to find thresholds that separate these objects accurately unless the object intensities considerably differ from the background. However, this is generally not the case in prostate images so most of the applications need more adequate probability models.

- Atlas-based techniques use anatomical atlases that depict prototypical locations and shapes of anatomical structures together with their spatial relations, as reference images to guide segmentation of new images. All the known atlas-based methods can be classified into single and multi-atlas-based segmentation. In general, the accuracy of the atlas-based segmentation depends on the accuracy of the atlas-to-target registration (i.e., the segmentation problem is reduced to the registration one). Also, the single-atlas-based segmentation could suffer from the possibly insufficient representation of the whole image population. The multi-atlas approach can overcome this drawback, but it requires a large number of atlas-to-target registration steps in order to produce the final segmentation.

2. Image Registration Methods

The majority of CADs assumes that the prostates (prostate contours) remain exactly the same from scan to scan. However, prostate contours may not always exactly match due to patient movement or breathing effects; therefore, accurate registration of the prostate in MR images is important to precisely analyze the transit of a contrast agent into the tissue, especially in the suspicious lesions. Accurate registration will also improve spatial correspondence of the prostate region and provide a one-on-one pixel match in all registered images, thus increasing the 
accuracy of diagnosis. To overcome the above-mentioned limitations, a novel noninvasive MRI-based framework for early diagnosis of prostate cancer is proposed. The proposed framework performs sequentially the following processing steps.

- Segmentation of the prostate from MR imaging based on a Maximum a Posteriori (MAP) estimate of a new likelihood function that accounts for both appearance features of the prostate and their 3D spatial voxel interactions , as well as a 3D shape prior.

- The integration of image registration approaches (nonrigid techniques), using geometric-based approaches (iso-contours) to improve spatial correspondence of regions-of-interest and provide a one-on-one pixel match in all registered images, thus increasing the accuracy of parameter estimation and/or diagnosis.

- KNN classifier to classify the prostate into benign or malignant based on three appearance features extracted from registered image.

\section{E. DISSERTATION ORGANIZATION}

This dissertation consists of six chapters, which can be summarized as follows.

- Chapter I present the background to adequately understand the anatomy of the prostate, prostate cancer, staging of the prostate cancer, and screening modalities. In addition, a brief summary of the basic contributions of the proposed research for diagnosing prostate cancer using DCE-MRI and DWI are also outlined. 
- Chapter II presents some basic concepts about prostate cancer and medical images. The current challenges of imaging the prostate in cancer.

- Chapter III presents an overview of the existing computational methods for diagnosing prostate cancer using MRI and outlines their strengths and weaknesses. Additionally, the state-of-the-art clinical applications and findings using these computational methods are covered.

- Chapter IV presents a novel, noninvasive framework in prostate application for early detection of prostate cancer using DCE-MRI.

- Chapter V presents a fully automated image-based framework for early detection of prostate cancer from DWI data.

- Chapter VI presents a general discussion about the presented research and its results, followed by the main conclusions and the possible areas for future work. 


\section{CHAPTER II}

\section{IMAGING THE PROSTATE IN CANCER}

Early detection of prostate cancer is crucial for the accurate diagnosis and institution of appropriate medical therapies, which can increase the survival rate of the patients [9]. However, a growing belief that nonlife threatening prostate cancers are being over-detected. Therefore, medical imaging techniques are favorable since they provide reliable information about the size and shape of the prostate gland and can localize the cancer foci. which would allow the determination of efficient treatments and enable more reliable methods of diagnosis and prognostication. Nowadays, medical imaging is a basic component in current medical diagnostic tools and has demonstrated the proven ability to detect prostate cancer without the associated deleterious side effects of invasive techniques. These noninvasive methods for prostate cancer diagnosis are based on acquiring scans of the prostate and analyzing these scans for cancer detection. To acquire scans of the prostate, different medical imaging techniques, such as Transrectal Ultrasound (TRUS), Single Photon Emission Computed Tomographic (SPECT), Positron Emission Tomography (PET), Magnetic Resonance Imaging (MRI), and Computed Tomography (CT), have been used (see Figure 7). Each of these image modalities has its own mechanism for providing relevant physiological information about the prostate as well as its own advantages and limitations. For example, CT is currently used for post-therapy evaluation by physicians to assess the effectiveness of treatment. However, it is expensive, uses radiation, and has poor contrast between soft tissues [19]. The SPECTs used in the detection of metastatic disease and are 
performed when symptoms for disease are present, such as bone pain. PET/CT allows differentiation between tumor and bowel in pelvis [20], it has limitations to distinguishing tumors from inflammation. As a result, TRUS and MRI are more commonly used in CAD systems for diagnosing prostate cancer.

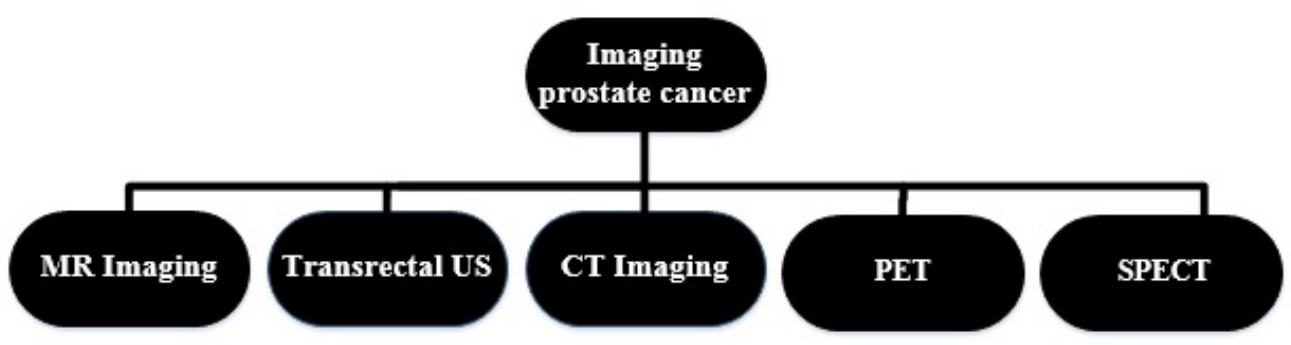

FIGURE 7: Schematic illustration of different medical image modalities for imaging the prostate in cancer.

The Transrectal Ultrasound (TRUS) imaging [21-23] is widely used for guided needle biopsy due to the real-time nature of the imaging system, ease of use, and portability (see Figure 8). However, TRUS images have a low signal to noise ratio (SNR) and detection of malignant tissues is difficult. Although,

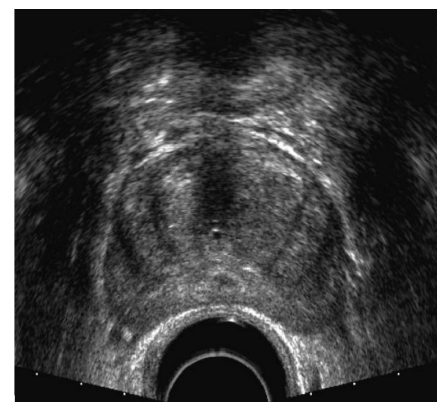

(a)

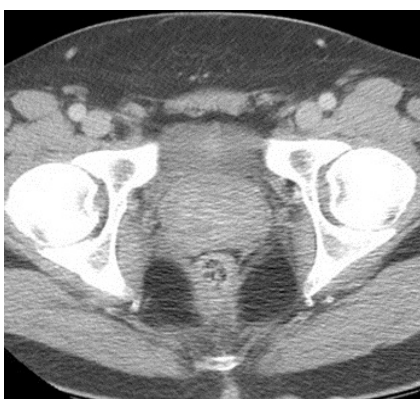

(b)

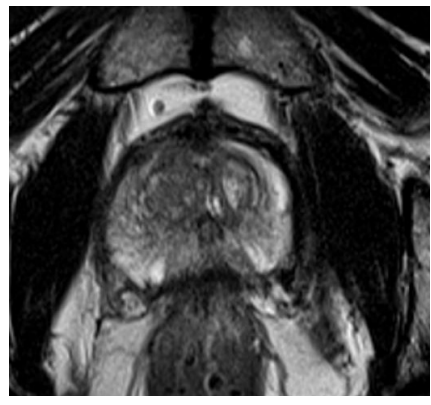

(c)

FIGURE 8: Different types of prostate medical images: (a) ultrasound image (Courtesy of [8]), (b) computed tomography (CT) image (Courtesy of [7]), and (c) $T_{2^{-}}$ weighted (MRI) image.

Magnetic Resonance Imaging (MRI) has been recently suggested for im- 
proved visualization and localization of the prostate. It provides valuable pathological and anatomical information [24]. Recently, new MRI modalities, such as Magnetic resonance Spectroscopy (MRS), Dynamic Contrast-Enhanced MRI (DCEMRI), and Diffusion-Weighted Magnetic Resonance Imaging (DW-MRI), have gained considerable attention as important tools for the early detection of prostate cancer. However, a side effect is an increase in image data that can be attributed to a number of factors including: increased image resolution, increased bits per volume image element (voxel), sampling in an increased number of dimensions, and an increased amount of tissue imaged. Although acquiring additional data provides a more complete view of the patient's physiology, it also creates a quantity of data that may be impossible for a physician to examine. For example, radiologists have traditionally dealt with only a few 2-D images (such as x-ray radiographs) per exam. However, now the capability exists for doctors to perform high resolution 3-D MRI scans of a patient. If many of these scans are taken at different times in order to monitor the progression of the patient's condition, the doctor is left with an immense 4-D dataset consisting of hundreds or thousands of images. Analysis consistency begins to suffer due to inter- and intra-observer variations. Since all the work presented in this dissertation mainly deals with MRI prostate data, an overview of different types of the MRI modality is given in the following section.

\section{A. MAGNETIC RESONANCE IMAGING}

Magnetic Resonance Imaging (MRI) has become the most powerful and central noninvasive tool for clinical diagnosis of diseases. The fundamental principle of MRI is based on the use of a strong static magnetic field in which the hydrogen nuclei (single proton) of water molecules in human tissues is aligned parallel to that field. Then, an external radio frequency (RF) pulse (wave) is applied to thun- 
paired magnetic spins (proton) aligned in the static magnetic field, making them spin in different directions [25]. The interaction between the RF and proton spins leads to periodic absorption and emission of energy. When the protons relax back to their lower energy (equilibrium) state, they release detectable signals (energy) that are spatially encoded and are used to construct the MR image.

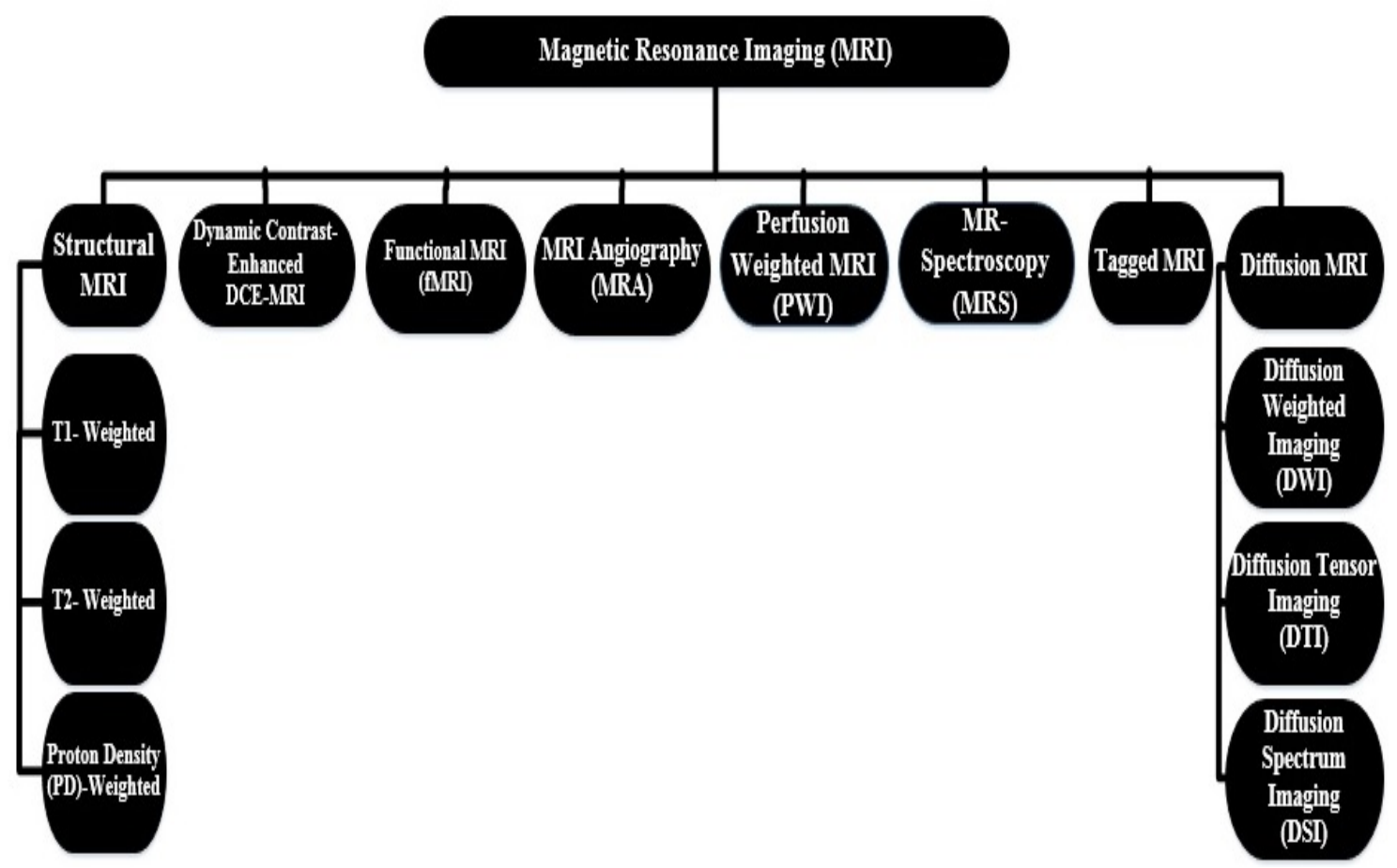

FIGURE 9: Different specialized MRI acquisition techniques.

Different types of tissues (muscle, fat, cerebral, spinal fluid, etc.) send back measurably different types of tissue-specific signals following the application of the same RF pulse. The contrast of an MR image is strongly dependent on the way the image is acquired. Different components of the scanned area can be highlighted using different preselected pulse sequences: strength, shape, and timing of the RF and gradient pulses (external fields).The most commonly-known specialized MRI techniques are shown in Figure 9. Generally, MRI can be used to acquire planar 2D images or 3D volumes [25]. Since this dissertation is concerned with building a 
MRI-based CAD system for the early diagnosis of prostate cancer, a brief overview of the basic principles of is presented in the next section.

\section{Structural MRI}

$T_{1}$ - and $-T_{2}$-weighted is a basic type of MRI in which most of the contrast between tissues is due to differences in tissue $T_{2}$ or $T_{1}$ values. Usually, $T_{2}$-weighted scans are used when contrast between fluid, abnormalities (e.g., tumors, inflammation, trauma), and the surrounding tissues is required. Therefore, it is the best MRI method for pathological details (see Figure 8 ). The $T_{2}$-weighted MRI modality has the ability to provide reliable information about the size and shape of the prostate gland. However, it is limited by unsatisfactory sensitivity and specificity for cancer detection and localization [26].

\section{Dynamic Contrast-Enhanced MRI (DCE-MRI)}

Although structural MRI provides excellent soft tissue contrast, it lacks functional information. Dynamic Contrast-Enhanced MRI (DCE-MRI) is a special MR technique that has the ability to provide superior information about the anatomy, function, and metabolism of target tissues. The DCE-MRI technique involves acquiring T1-weighted volume images before and at several time points after the injection of a contrast agent. The main role of the DCE-MRI is to increase the image contrast of anatomical structures (e.g., blood vessels) that are not easily visualized by the alteration of the magnetic properties of water molecules in their vicinity. This in turns improves the visualization of tissues, organs, and physiological processes. The DCE-MRI takes advantage of the network of microvascular structures associated with cancerous tumors. Specifically, the DCE-MRI procedure allows 
for the observation and quantification of vascular permeability and vascular density curves or time-intensity curves as shown in Figure 11. Typical examples of dynamic MRI time series data of the prostate are shown in Figure 10.
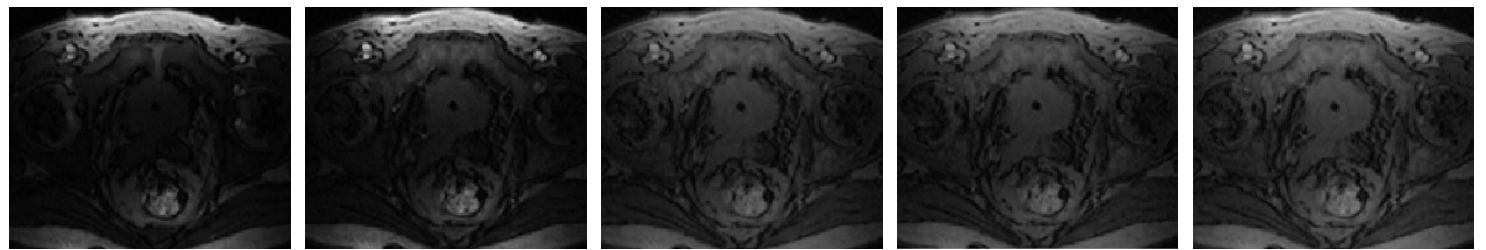

FIGURE 10: MRI Prostate images taken at different time points post the adminstration of the contrast agent showing the change of the contrast as the contrast agent perfuse into the prostate tissues.

(a)

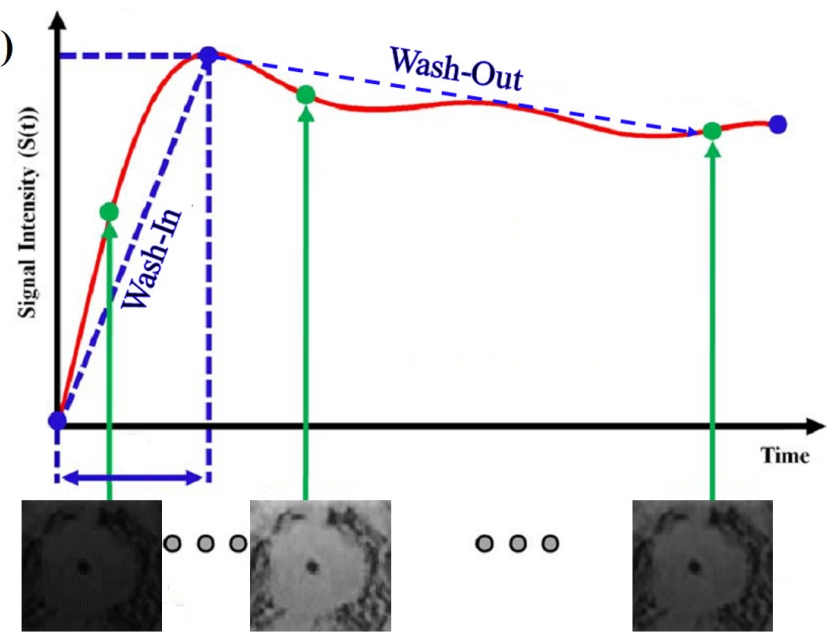

(b)

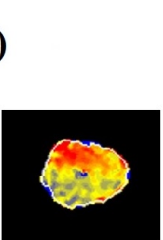

High Perfusion

Low Perfusion

FIGURE 11: (a) A typical time-intensity curve of the average intensity of the prostate measured before and after contrast agent adminstration. The curve illustrates typical perfusion-related indexes that can be estimated and used for diagnosis of the prostate cancer. (b) Prostate colored by wash-in and wash-out 
3. Magnetic Resonance Spectroscopy Imaging (MRSI)

Magnetic Resonance Spectroscopy Imaging (MRSI) allows the evaluation of the metabolic activity within the prostate gland by assessing the quantities of different metabolites. Within the prostate, the most commonly detected metabolites are choline, creatine, polyamines and citrates. In a healthy prostate, there are low levels of choline and high levels of citrates; the opposite is observed in patients with prostate cancer. Polyamines are increased in benign prostatic hyperplasia and reduced in cancer (see Figure 12). A ratio of choline-plus-creatine to citrate has been used to help differentiate benign from malignant lesions [27].

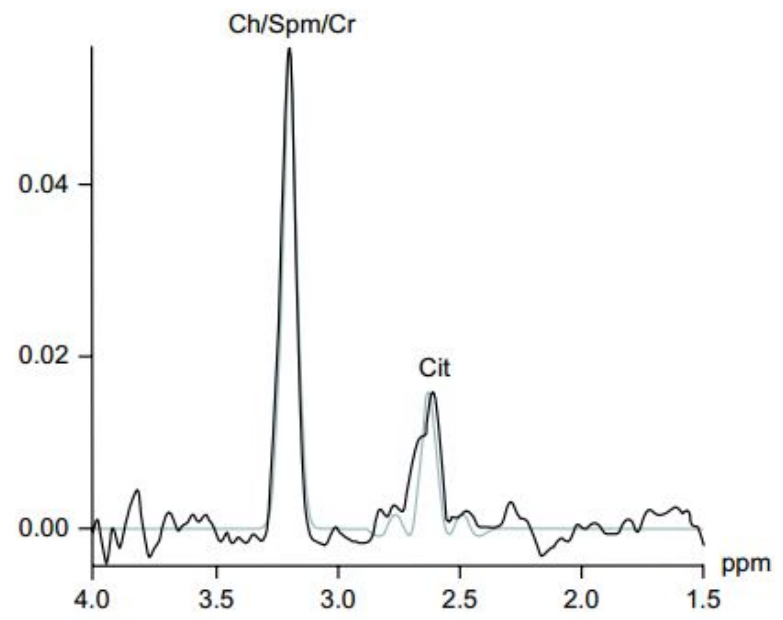

FIGURE 12: Examples of MRS. There is a single choline, polyamine, and creatine peak.

4. Diffusion-Weighted Imaging (DWI)

DWI is designed to obtain images whose contrast depends on the differences in water molecule mobility. This is achieved during data acquisition by adding diffusion magnetic field gradients. The degree of diffusion weighting of the sequence, expressed as the b-factor (in $s / \mathrm{mm}^{2}$ ), depends on the amplitude of 
the field gradient, time of application, and time interval between the magnetic field gradients. A typical DWI-MRI for the prostate is shown in Figure 13. DWI is a well-established MRI method that has been successfully used for tumor localization and diagnosis [28].

(a)
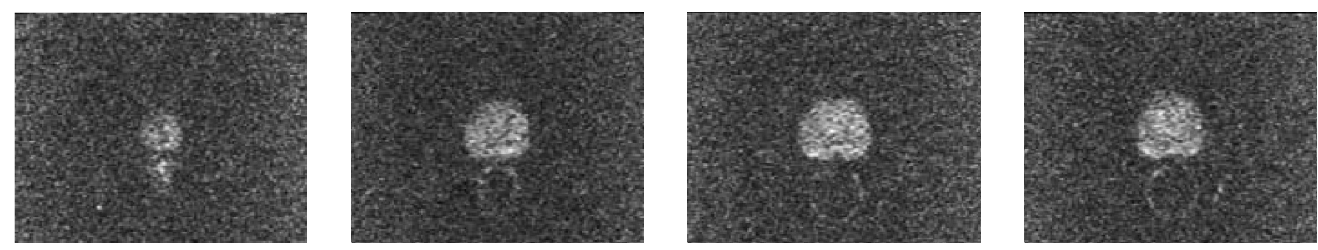

(b)
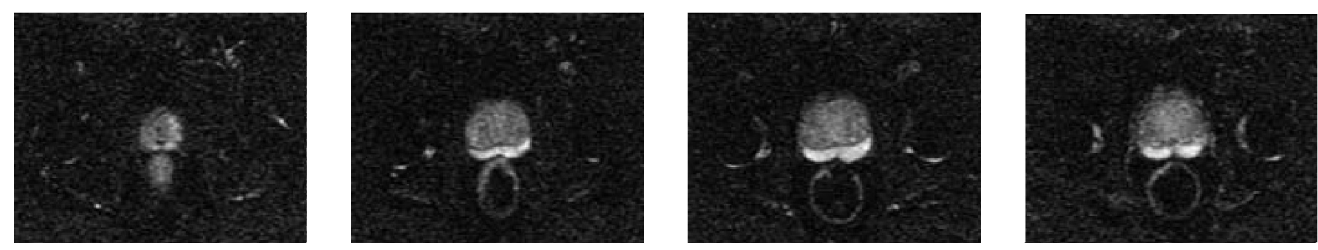

FIGURE 13: Diffusion MR images for the prostate at (a) $b$-value of $0 \mathrm{~s} / \mathrm{mm}^{2}$ and (b) b-value of $800 \mathrm{~s} / \mathrm{mm}^{2}$.

\section{B. QUANTIFICATION OF PROSTATE CANCER USING MR IMAGES}

The use of MRI medical images for prostate cancer has been increased in recent years due to its ability to provide superior anatomical and functional information about the tumor classification. The goal of this work is to evaluate the diagnostic value of an imaging protocol that uses Dynamic Contrast-Enhanced MRI (DCE-MRI) and Diffusion-Weighted Imaging (DWI) in patients with suspicious prostate cancer and to determine if additional information, provided by DWI, improves the diagnostic value of prostate MRI [29]. In this dissertation, the proposed models have been applied to both imaging protocols.

a. Early Detection of Prostate Cancer using (DCE-MRI) The DCE-MRI has demonstrated the promise for early detection of prostate cancer [30]. The DCEMRI process begins with several MRI scans which are used to establish a baseline 
in image intensity. These scans are performed without the administration of contrast enhancing agents so that the tissue's non-enhanced image intensity can be established. In the next stage, a contrast agent (such as Gadolinium) is administered intravenously. At this point, MRI scans are performed at regular intervals. The contrast agent flows throughout the body, including the tissues being imaged. Malignant areas of increased microvascular growth have increased vascular leakage; therefore, in these regions more contrast agent passes between the vascular system and the extravascular tumor tissue [30-32]. The observed dynamics of the buildup and washout of the contrast agent can help characterize the micro-vessel density and porosity. Repeated MRI scans continue until the body filters most of the contrast agent from the blood stream via the kidneys. A signal intensity versus time curve is created for each voxel in the volume, and local exchange model parameters, inferred from these curves, are used by radiologists to detect and classify malignant lesions. Currently, manual DCE-MRI analysis is performed using estimated pharmacokinetic parameters which quantify the movement of the contrast agent into and out of tissues. These parameters are color-coded and superimposed on a pre-contrast MR image. The radiologists then manually outline the lesions in the superimposed images using a computer mouse. Once segmented, the average time vs signal intensity plot may be observed and the lesion is classified by matching the resulting plot with theoretical plots for specific lesion types.

The proposed work in this dissertation seeks to provide a new noninvasive system that possesses the ability to facilitate the accurate analysis of the perfusion of a CA in the prostate tissue in an effort to extract perfusion parameters that distinguish between malignant and benign prostate cancers. The innovation in this case study is a CAD system that integrates stochastic approaches using new Markov-Gibbs random field (MGRF) energy models and the geometric approaches to accurately separate the prostate from the surrounding tissues, after handling the global and local motion, in order to optimally distinguish between malignant and 
benign prostate cancers (see Figure 14).

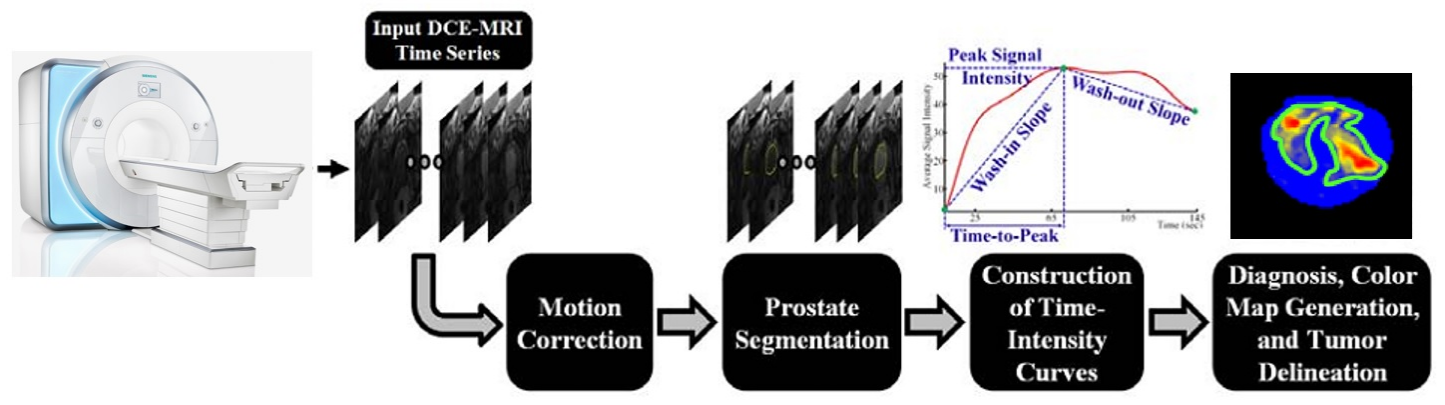

FIGURE 14: A block diagram of the proposed CAD system for the early diagnosis of prostate cancer using DCE-MRI. The time series data is first corrected for motion artifacts. Then, the prostatic tissue is segmented and the agent kinetic curves are constructed. Finally, perfusion-related parameters are extracted from the kinetic curves and are used for the classification of both malignant and benign cases.

\section{b. Diffusion-Weighted MRI Diffusion-Weighted MRI (DWI),}

a non-invasive technique in which molecular motion of water is measured in biological tissues, is now used in the detection of prostate cancer as an adjunct to $T_{2}$-weighted [33]. The Apparent Diffusion Coefficient (ADC) calculated from DWI in prostate cancer showed that the mean $\mathrm{ADC}$ for malignant prostate is lower than the mean $\mathrm{ADC}$ in the non-malignant prostatic tissue [34-39]. In recent years, a growing number of clinical studies have evaluated the utility of DWI, either in combination with or in comparison with other MRI techniques, for the detection of prostate cancer. These studies have reported various sensitivities and specificities of cancer diagnosis [34,40-43]. In this dissertation, a comprehensive framework for detection of prostate cancer is proposed based on DWI, see Figure 15. The proposed diagnostic approach segments the prostate from the surrounding anatomical structures based on a Maximum a Posteriori (MAP) estimate of a new log-likelihood function after handling the global and local motions. Then, three 
appearance features are extracted and used for the evaluation of the potential prostate tumor. In addition, the tumor boundaries are determined using a level set deformable model controlled by the diffusion information and the spatial interactions between the prostate voxels, see Figure 16.

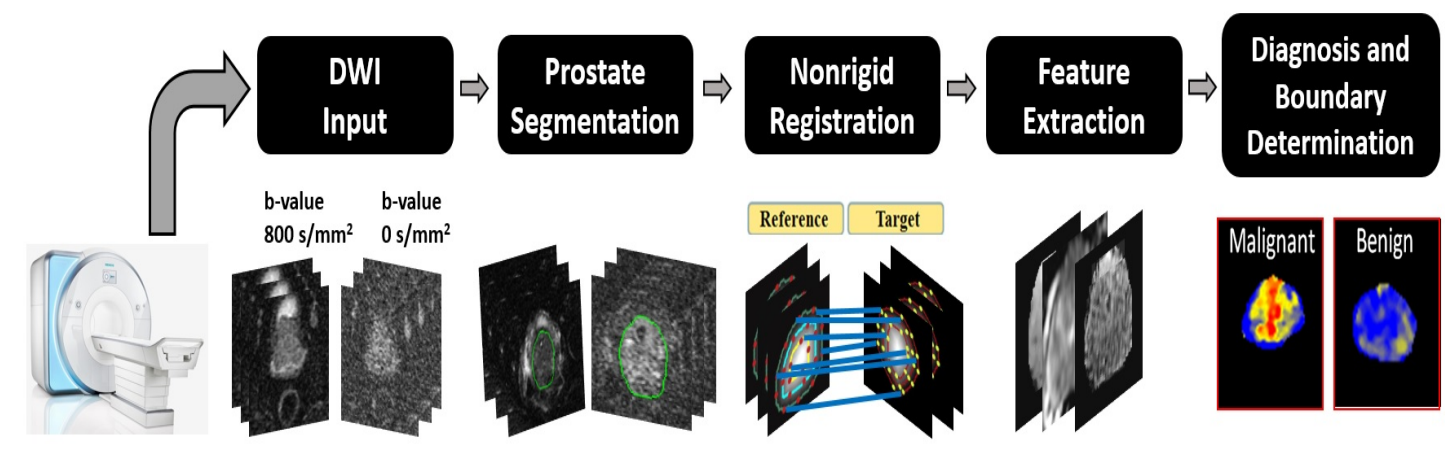

FIGURE 15: Flowchart of the proposed CAD system for automatic detection of cancer from 3D DWI. An automatic prostate segmentation method is applied to isolate the prostate from the surrounding anatomical structures. Following segmentation, a non-rigid registration approach is employed to account for any local deformation that could occur in the prostate during the scanning process. Three features are extracted from registered diffusion data. The features are summarized by a supervised classifier to calculate the likelihood of malignancy. 


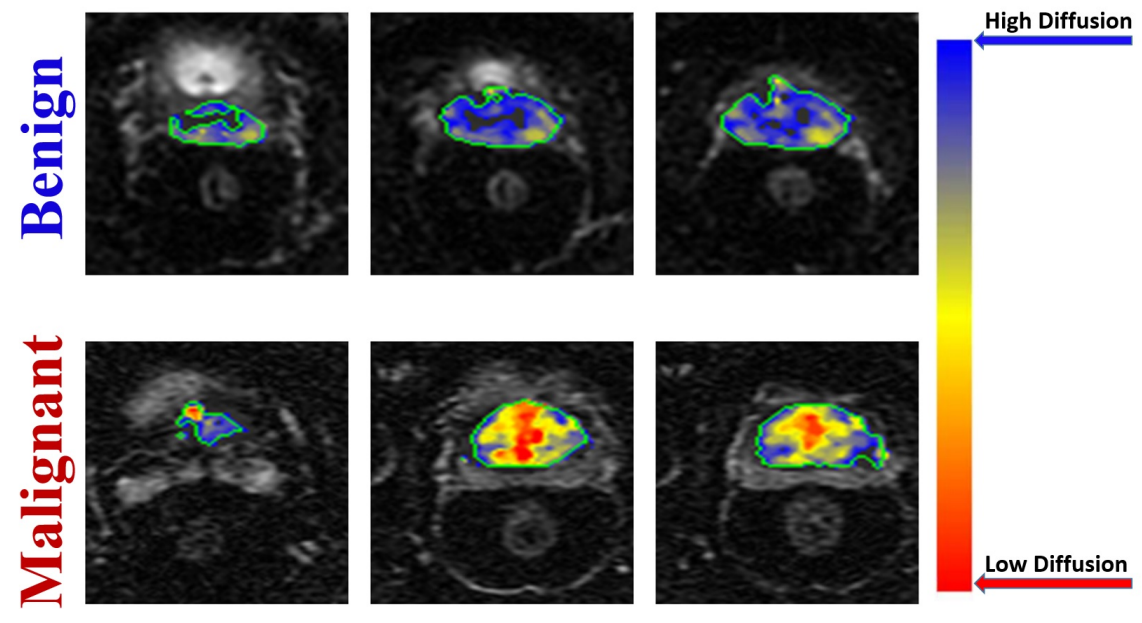

FIGURE 16: Pixel-wise parametric map display of the diffusion information and the spatial interactions between the prostate voxels. The red and blue hues of each color scale correspond to enhanced and reduced perfusion, respectively. 


\section{CHAPTER III}

\section{IMAGE BASED RELATED WORK FOR EARLY DIAGNOSES OF PROSTATE CANCER}

This chapter discusses the developed non-invasive state-of-the-art Computer Aided Diagnosis (CAD) systems for prostate cancer based on analyzing different types of Magnetic Resonance Imaging (MRI), e.g., $T_{2}$-MRI, Diffusion Weighted Imaging (DWI), Dynamic Contrast Enhanced (DCE)-MRI, and multiparametric MRI, focusing on their implementation, experimental procedures, and reported outcomes. Furthermore, the chapter addresses the limitations of the current prostate cancer diagnostic techniques, outlines the challenges that these techniques face, and introduces the recent trends to solve these challenges. Prostate segmentation from MRI images, i.e., the delineation of prostate borders from the surrounding tissues, is a basic step in any noninvasive CAD system for early detection of prostate cancer. However, accurate delineation of prostate borders in MR images is a challenge due to: large variations of prostate shapes within a specific time series as well as across subjects; the lack of strong edges and diffused prostate boundaries; and the similar intensity profile of the prostate and surrounding tissues. Although manual outlining of the prostate border enables the prostate volume to be determined, it is time consuming and observer dependent. Moreover, traditional edge detection methods [24] are unable to extract the correct boundaries of the prostate since the gray level distributions of the prostate and the surrounding organs are hardly distinguishable. To overcome this limitation, most successful known approaches have addressed the segmentation challenges by incorporating 
the prostate appearances and shapes into their segmentation techniques. In particular, an automated framework by Allen et al. [44] was proposed for 3D prostate segmentation that consists of two steps. voxel classification is performed based on Gaussian probabilities of grey level. Then, a statistical shape model is used to segment the prostate region. A hybrid 2D/3D active shape model (ASM)-based methodology for global optimal segmentation of the 3D MRI prostate data was proposed by Zhu et al. [45]. Iterative segmentation was performed by a 2D ASM search on each slice, then the final surface is reconstructed from the $2 \mathrm{D}$ search results and updated by re-estimating the parameters of the 3D probabilistic shape model. Klein et al. $[8,46]$ presented an atlas-based segmentation approach that utilized a localized version of mutual information (MI) to extract the prostate from MR images. The segmentation of the prostate is obtained as the average of the bestmatched registered atlas set to the test image (image to be segmented).Flores-Tapia et al. [47] proposed a semi-automated edge detection technique for MRI prostate segmentation. In their framework, the prostate borders were detected by tracing four manually-selected reference points on the edge of the prostate using a static Wavelet transform [48] to locate the prostate edges. Toth et al. [49] presented an algorithm for the automatic segmentation of the prostate in multi-modal MRI. Their algorithm starts by isolating the region of interest (ROI) from MRS data. Then, an ASM within the ROI is used to obtain the final segmentation. A semi-automated approach by Vikal et al. [50] used a priori knowledge of prostate shape to detect the contour in each slice and then refined them to form a 3D prostate surface. Firjani et al. [51] proposed a MAP based framework that combines a graph-cut approach and three image features (grey-level intensities, spatial interactions between the prostate pixels, and a prior shape model) for 2D DCE-MRI prostate segmentation. Their method was later extended in [52] to allow for 3D segmentation from DCEMRI volumes. It utilized both a 3D MRF to model the spatial interaction between the prostate voxels and a $3 \mathrm{D}$ shape prior. 
An unsupervised segmentation method was proposed by Liu et al. [53] for the segmentation of MR prostate images. A level set deformable model was employed and was guided by an elliptical prostate shape prior and intensity gradient was employed to refine the initial results obtained by Otsu thresholding [54]. A maximum A posteriori (MAP)-based framework was proposed by Makni et al. [55] to perform automated 3D MRI prostate segmentation. Their framework combined graylevel, contextual information regarding voxels neighborhoods using MRF, and statistical shape information to find optimum segmentation based on Bayesian a posteriori classification, estimated with the iterative conditional mode (ICM) algorithm. Liu et al. [56] proposed an automated approach that utilized fuzzy MRF modeling for prostate segmentation from multi-parametric MRI. Their framework exploited $T_{2}$-weighted image intensities, pharmacokinetic (PK) parameter kep , and apparent diffusion coefficient (ADC) values in a Bayesian approach to label prostate pixels as cancerous or non-cancerous. The labeled pixels are then clustered using the k-means algorithm. The system had a specificity of $89.58 \%$, sensitivity of $87.50 \%$, accuracy of $89.38 \%$, and a DSC of $62.2 \%$. A similar approach was developed by Artan et al. [57] and located cancerous regions using cost-sensitive support vector machine (SVM). Prostate segmentation was performed using a conditional random field and the same three features in [56] were utilized for classification. The DSC for prostate localization and segmentation was $0.46 \pm 0.26$, and the area under the receiver operator characteristic (ROC) curves $\left(A_{z}\right)$ of the classification was $0.79 \pm 0.12$. Ozer et al. [58] also developed a technique that directly segmented prostate cancers using the same three features in $[56,57]$. Both the SVM and relevance vector machine (RVM) [59] classifiers were used and the system showed a specificity of 0.78 and a sensitivity of 0.74 for RVM and 0.74 and 0.79 for SVM. Gao et al. [60] proposed a shape-based technique that utilized point cloud registration of the MR images before segmenting the prostate. The final prostate border is obtained by minimizing a cost functional that incorporated both the local image statistics as well as the learned shape prior. Martin et al. [61] developed 
an atlas-based approach for segmenting the prostate from 3D MR images by mapping a probabilistic anatomical atlas to the test image. The resulting map is used to constrain a deformable model-based segmentation framework. Recently, Dowling et al. [62] proposed an automated framework that combined dynamic multiatlas label fusion methods. They employed the diffeomorphic demons method for the nonrigid registration using the selective and iterative method for performance level estimation (SIMPLE) technique [63]. In their framework, a pre-processing step for bias field correction, histogram equalization, and anisotropic diffusion smoothing was employed. Ghose et al. [64] proposed a probabilistic graph-cutbased framework for prostate segmentation based on the fusion of the posterior probabilities determined with a probabilistic atlas and a supervised random forest learning framework. An automated technique that first applied global registration to the prostate MRI data followed by an active appearance model (AAM) based segmentation of the prostate tissue was proposed by Ghose et al. [65]. Table 2 summarizes the reviewed methodologies for prostate segmentation and registration with the validation data sets and achieved performance for each study. However, in most of these approaches the segmentation and registration reliability is not very high due to the following reasons: $(i)$ parametric shape models fail in the presence of large gray-level variability across subjects and time; (ii) edge detection methods are not suitable for discontinued objects; (iii) deformable models tend to fail in the case of excessive noise, poor image resolution, diffused boundaries or occluded objects if they do not incorporate a priori models (e.g., shape and appearance). In addition, most of the motion correction models account only for the global motion and do not take into account the local motion of the prostate due to transmitted respiratory and peristaltic effects. Furthermore, the existing local motion correction methods are intensity-based techniques, which are prone to nonlinear intensity variations over the time series and perform poorly in pre-contrast images. Also, local motion correction methods register the original grey level data without any prior segmentation; therefore, they do not guarantee voxel-on-voxel 
matches of the registered perfusion data.

The state-of-the-art CAD systems extract several features from MR image modality for classifying a prostate region as cancerous or noncancerous. Several CAD systems in the literature have used multi-parametric MRI, a combination of multiple MRI modalities, to increase the number and quality of the features that the systems can utilize. A summary of the the common features extracted from each of these MRI modalities as well as the basic systems along with their computational methods, validation data sets, and validation accuracy are given in Table 3 . To the best of our knowledge, the first semi-automated computerized MRI-based CAD system for prostate cancer diagnosis was developed by Chan et al [66]. In their study, multi-modal MRI $\left(T_{2}\right.$-weighted, $T_{2}$-mapping, and line scan diffusion imaging) were used to estimate malignancy likelihood in the PZ of the prostate. Both statistical maps and textural features were obtained and a SVM and a linear discriminant analysis (LDA) classifiers were employed for the classification. Their systems resulted in an $A_{z}$ of $0.761 \pm 0.043$ and $0.839 \pm 0.064$, for SVM and LDA respectively. Madabhushi et al [67] proposed an automated CAD system for detecting prostatic adenocarcinoma from MR prostate images. In their method, multiple image features, including gray levels statistics (intensity values, mean, and standard deviation), intensity gradient, and Gabor filter features, were used for classifying groups of pixels as tumors. A K-nearest neighbor classifier and Bayesian conditional densities were used for classification, and the system achieved an $A_{z}$ of 0.957. A study by Engelbrecht et al. [68] evaluated which MRI parameters would result in optimal discrimination of prostatic carcinoma from normal PZ and CZ of the prostate. Using the ROC curves, their study concluded that the relative peak enhancement was the most accurate perfusion parameter for cancer detection in the PZ and CZ of the gland. A semiautomated CAD system by Kim et al. [27] demonstrated that parametric imaging of the wash-in rate was more accurate for the detection of prostate cancer in the PZ than was $T_{2}$-weighted imaging 
alone. However, they also observed significant overlap between the wash-in rate for cancer and normal tissue in the TZ. Fütterer et al. [69] developed a CAD system to compare the accuracies of $T_{2}$-weighted MRI, DCE-MRI, and MRS imaging for prostate cancer localization. The results showed higher accuracy in DCE-MRI than were achieved with $T_{2}$-weighted MRI in prostate cancer localization. A similar study was conducted by Rouvièere et al. [70] for the detection of postradiotherapy recurrence of prostate cancer. Their study also concluded that DCE-MRI possesses the ability to depict the intraprostatic distribution of recurrent cancer after therapy more accurately and with less inter-observer variability than $T_{2}-$ weighted MRI. Puech et al. [71] developed a semiautomated dynamic MRI-based CAD system for the detection of prostate cancer. Candidate lesion ROIs were selected either manually or by using a region growing technique initiated by a user-selected seed point. Lesions are classified as benign, malignant or indeterminate based on the analysis of the median wash-in and wash-out values. Their CAD system demonstrated a sensitivity and specificity of $100 \%$ and $45 \%$ for the PZ, and sensitivity and specificity of $100 \%$ and $40 \%$ for the TZ. Ocak et al. [72] developed a CAD system using PK analysis for prostate cancer diagnostics in patients with biopsy-proven lesions. In their framework, four PK parameters $\left(K^{\text {trans }}, k^{e p}, v_{e}\right.$, and the area under the gadolinium concentration curve) were determined and compared for cancer, inflammation, and healthy peripheral. Their results showed improvement in prostate cancer specificity using the $K^{\text {trans }}$ and $k^{e p}$ parameters over that obtained using conventional $T_{2}$-weighted MRI. An automated DCE-MRI CAD system for prostate cancer detection was proposed by Viswanath et al. [73]. Prostate borders were segmented using an ASM, and a nonrigid registration scheme (affine and thin plate spline) was employed to map the whole mount histological sections onto corresponding 2D DCE-MRI. In order to classify prostate tissue, a local linear embedding approach [74] was used to create a feature vector using local neighborhood intensities. Then, a k-means clustering approach was used for the classification and the system achieved an accuracy of about $77 \%$. Their framework was later 
extended in [75] by combining $T_{2}-$ weighted features and DCE-MRI functional features. The system validation showed that the integration of both modalities ( $A_{z}$ of 0.815$)$ has a better performance of either individual modalities $(0.704$ for $T_{2}$-weighted MRI and 0.682 for DCE-MRI). A semi-automated framework by Vos et al. [76] classified prostate lesions using quantitative PK maps and $T_{1}$ estimates. PK features were extracted from a user-defined ROI around the prostate and a SVM was used to estimate the likelihood of malignancy. Based on the ROC analysis, the reported results showed that the system had an accuracy of $83 \%$ in the classification of the ROIs with abnormal enhancement patterns in the PZ. Ampeliotis et al. [77] proposed a semi-automated multi-parametric CAD system that used T 2 -weighted and DCE-MRI. The $T_{2}$-weighted pixel intensities and the four lowfrequency coefficients of the discrete cosine transform were used as features and probabilistic neural networks were employed as the classifier. Based on the ROC analysis $\left(A_{z}\right.$ of 0.898$)$, their study concluded that the fused $T_{2}$-weighted and dynamic MRI features outperform that of either modalitys features alone. A similar CAD system was proposed by Litjens et al. [78] that employed an ASM to segment the prostate. In order to classify the segmented prostate voxels, the ADC, $K^{\text {trans }}$, and $k^{e p}$ parameters were estimated and a SVM classifier with a radial basis function kernel was used. The validation results showed a sensitivity of $74.7 \%$ and 83.4\% with seven and nine FPs per patient, respectively. Vos et al. [75] utilized an automated CAD system for the detection of prostate cancer. Just as in [78], the prostate was segmented using an ASM-based technique. Then, multiple ROIs were located within the segmented prostate using peak and mean neighborhood intensity and ADC values. These values and the differences between the peak and the mean were again used as features for ROI classification. In addition, the 25 percentile $T_{2}$, 25 percentile ADC, 25 percentile wash-out, 50 percentile $T_{1}, 75$ percentile $K^{\text {trans }}$, and 75 percentile $v_{e}$ were used as features. The resulting feature vector was classified using an LDA classifier. This system had an $A_{z}$ of 0.830 .20 . A maximum $A_{z}$ of 0.88 was reported for high-grade tumors, but the system had dif- 
ficulty classifying lower grade tumors, achieving a maximum $A_{z}$ of 0.74 . Another semi-automated multi-parametric system by Peng et al. [79] utilized $T_{2}$-weighted, DCE-MRI and DWI. Candidate features, including the T 2 -weighted intensity skew, the $K^{\text {trans }}$, and the average and 10th percentile ADC, were calculated from a manually-selected ROI. Then, an LDA classifier was used to differentiate prostate cancer from normal tissue in those ROIs. Their CAD system concluded that the best diagnostic performance $\left(A_{z}\right.$ of $0.95 \pm 0.02$, SEN of 0.82 , and SPE of 0.953$)$ is obtained by combining the $10^{\text {th }}$ percentile ADC, average ADC, and $T_{2}$-weighted intensity skewness features.

TABLE 2: Summary of the discussed prostate segmentation and registration techniques and their experimental performance. Note that DIM and AL stand for data dimension (i.e., 2D, 3D, or 4D) and the automation level (i.e., automated or semiautomated), respectively.

\begin{tabular}{|c|c|c|}
\hline Study & DIM, AL, and Methods & Data and Performance \\
\hline $\begin{array}{l}\text { Allen et } \\
\text { al. [44] }\end{array}$ & $\begin{array}{l}\text { - } 3 \mathrm{D} \\
\text { - Automated } \\
\text { - } \text { ASM-based segmentation }\end{array}$ & $\begin{array}{l}\text { - } 22 \text { data sets } \\
\text { - MAD: } 2.8 \pm 0.82\end{array}$ \\
\hline $\begin{array}{l}\text { Klein et } \\
\text { al. [46] }\end{array}$ & $\begin{array}{l}\text { - } 3 \mathrm{D} \\
\text { - Automated } \\
\text { - Affine + B-Splines Registra- } \\
\text { tion } \\
\text { - Atlas-based segmentation }\end{array}$ & $\begin{array}{l}\text { - } 38 \text { data sets } \\
\text { - Median DSC: } 0.82\end{array}$ \\
\hline $\begin{array}{l}\text { Zhu et } \\
\text { al. [45] }\end{array}$ & $\begin{array}{l}\text { - } 3 \mathrm{D} \\
\text { - Automated } \\
\text { - Hybrid 2D+3D ASM }\end{array}$ & $\begin{array}{l}\text { - } 26 \text { data sets (288 slices) } \\
\text { - RMSD: } 5.481 \pm 2.91\end{array}$ \\
\hline $\begin{array}{l}\text { Flores- } \\
\text { Tapia et } \\
\text { al. [47] }\end{array}$ & $\begin{array}{l}\text { - 3D } \\
\text { - Semi-automated } \\
\text { - Edge detection }\end{array}$ & $\begin{array}{l}\text { - } 1 \text { data set (19 slices) } \\
\text { - DSC: } 0.93 \pm 0.005\end{array}$ \\
\hline $\begin{array}{l}\text { Toth et } \\
\text { al. [49] }\end{array}$ & $\begin{array}{l}\text { - } 3 \mathrm{D} \\
\text { - Automated } \\
\text { - Unsupervised spectral clus- } \\
\text { tering } \\
\text { - ASM-based segmentation }\end{array}$ & $\begin{array}{l}\text { - } 150 \text { slices } \\
\text { - Average OR: } 0.83 \text {, average } \\
\text { SEN: 0.89, average SPE: } \\
0.86 \text {, and average PPV: } \\
0.93\end{array}$ \\
\hline $\begin{array}{l}\text { Klein } \\
\text { al. [8] }\end{array}$ & $\begin{array}{l}\text { - } 3 \mathrm{D} \\
\text { - Automated } \\
\text { - Affine + B-splines registra- } \\
\text { tion } \\
\text { - Atlas matching }\end{array}$ & $\begin{array}{l}\text { - } 50 \text { slices } \\
\text { - Median DSC: } 0.85\end{array}$ \\
\hline
\end{tabular}


TABLE 2 - continued from the previous page

\begin{tabular}{|c|c|c|}
\hline Study & DIM, AL, and Methods & Data and Performance \\
\hline $\begin{array}{ll}\text { Vikal } & \text { et } \\
\text { al. [50] } & \end{array}$ & $\begin{array}{l}\text { - } 3 \mathrm{D} \\
\text { - Semi-automated } \\
\text { - } \mathrm{ASM}\end{array}$ & $\begin{array}{l}\text { - } 3 \text { data sets (39 slices) } \\
\text { - DSC: } 0.93 \pm 0.3 \text { and MAD: } \\
\text { 2.00 } \pm 0.6(\mathrm{~mm})\end{array}$ \\
\hline $\begin{array}{ll}\text { Makni } & \text { et } \\
\text { al. [55] } & \end{array}$ & $\begin{array}{l}\text { - } 3 \mathrm{D} \\
\text { - Automated } \\
\text { - } \text { ASM }\end{array}$ & $\begin{array}{l}\text { - } 12 \text { data sets } \\
\text { - Mean HD: } 9.62(\mathrm{~mm}), \mathrm{GD}: \\
2.39(\mathrm{~mm}), \mathrm{OR}: 0.84, \mathrm{VPC}: \\
\text { 0.90, and DSC: } 0.91\end{array}$ \\
\hline $\begin{array}{l}\text { Liu et } \\
\text { al. [53] }\end{array}$ & $\begin{array}{l}\text { - } 2 \mathrm{D} \\
\text { - Automated } \\
\text { - Level-set-based segmenta- } \\
\text { tion }\end{array}$ & $\begin{array}{l}\text { - } 10 \text { data sets } \\
\text { - DSC: } 0.91 \pm 0.03\end{array}$ \\
\hline $\begin{array}{l}\text { Ozer et al. } \\
{[58]}\end{array}$ & $\begin{array}{l}\text { - } 2 \mathrm{D} \\
\text { - Automated } \\
\text { - SVM and RVM }\end{array}$ & $\begin{array}{l}\text { - } 20 \text { data sets } \\
\text { - SPE: } 0.78, \text { SEN: } 0.74 \text {, and } \\
\text { DSC: } 0.51 \text { for RVM } \\
\text { - SPE: } 0.74, \text { SEN: } 0.79 \text { and } \\
\text { DSC: } 0.52 \text { for SVM }\end{array}$ \\
\hline $\begin{array}{l}\text { Artan et } \\
\text { al. [57] }\end{array}$ & $\begin{array}{l}\text { - } 2 \mathrm{D} \\
\text { - Automated } \\
\text { - Cost-sensitive } \\
\text { random field }\end{array}$ & $\begin{array}{l}\text { - } 21 \text { data sets } \\
\text { - } A_{z}: 0.790 \pm 0.12 \text { and DSC: } \\
0.46 \pm 0.26\end{array}$ \\
\hline $\begin{array}{l}\text { Liu } \\
\text { al. [56] }\end{array}$ & $\begin{array}{l}\text { - } 3 \mathrm{D} \\
\text { - Automated } \\
\text { - fuzzy MRFs }\end{array}$ & $\begin{array}{l}\text { - } 11 \text { data sets } \\
\text { - SPE: 0.896, SEN: 0.894, } \\
\text { and DSC: } 0.622\end{array}$ \\
\hline $\begin{array}{ll}\text { Gao } & \text { et } \\
\text { al. [60] } & \end{array}$ & $\begin{array}{l}\text { - } 3 \mathrm{D} \\
\text { - Automated } \\
\text { - Affine registration } \\
\text { - Level set segmentation }\end{array}$ & $\begin{array}{l}\text { - } 48 \text { data sets } \\
\text { - DSC: } 0.84 \pm 0.03 \text { and } 95 \% \\
\text { HD: } 8.10 \pm 1.50(\mathrm{~mm})(33 \\
\text { data sets) } \\
\text { - DSC: } 0.82 \pm 0.03 \text { and } 95 \% \\
\text { HD: } 10.22 \pm 4.03 \text { (mm) (15 } \\
\text { public data sets) }\end{array}$ \\
\hline $\begin{array}{l}\text { Martin et } \\
\text { al. [61] }\end{array}$ & $\begin{array}{l}\text { - } 3 \mathrm{D} \\
\text { - Automated } \\
\text { - Affine + Multi-resolution } \\
\text { Demons registration } \\
\text { - ASM }\end{array}$ & $\begin{array}{l}\text { - } 36 \text { data sets } \\
\text { - Median DSC: } 0.86 \text { and } \\
\text { Average surface error: } \\
2.41 \mathrm{~mm}\end{array}$ \\
\hline $\begin{array}{l}\text { Dowling et } \\
\text { al. [62] }\end{array}$ & $\begin{array}{l}\text { - } 3 \mathrm{D} \\
\text { - Automated } \\
\text { - Diffeomorphic demons regis- } \\
\text { tration } \\
\text { - Atlas-based segmentation }\end{array}$ & $\begin{array}{l}\text { - } 50 \text { data sets } \\
\text { - Median DSC: } 0.86 \text { and Av- } \\
\text { erage surface error: } 2.0 \\
(\mathrm{~mm})\end{array}$ \\
\hline \multicolumn{3}{|c|}{ continued on the next page... } \\
\hline
\end{tabular}


TABLE 2 - continued from the previous page

\begin{tabular}{|c|c|c|}
\hline Study & DIM, AL, and Methods & Data and Performance \\
\hline $\begin{array}{l}\text { Ghose et } \\
\text { al. [64] }\end{array}$ & $\begin{array}{l}\text { - } 3 \mathrm{D} \\
\text { - Semi-automated } \\
\text { - Affine + nonrigid Demon } \\
\text { registration } \\
\text { - Graph-cut segmentation }\end{array}$ & $\begin{array}{l}\text { - } 15 \text { data sets } \\
\text { - DSC: } \quad 0.91 \pm 0.04 \quad \text { and } \\
\text { 95\%HD: } 4.69 \pm 2.62(\mathrm{~mm})\end{array}$ \\
\hline $\begin{array}{l}\text { Ghose et } \\
\text { al. [65] }\end{array}$ & $\begin{array}{l}\text { - } 2 \mathrm{D} \\
\text { - Automated } \\
\text { - Affine registration } \\
\text { - AAM-based segmentation }\end{array}$ & $\begin{array}{l}\text { - } 15 \text { data sets } \\
\text { - DSC: } 0.88 \pm 0.11 ; \quad \text { HD: } \\
3.38 \pm 2.81 \quad(\mathrm{~mm}), \quad \text { and } \\
\text { MAD: } 1.32 \pm 1.53\end{array}$ \\
\hline AMM: & \multirow{3}{*}{\multicolumn{2}{|c|}{$\begin{array}{l}\text { Active appearance model. } \\
\text { Active shape model. } \\
\text { Dice similarity coefficient: } \mathrm{DSC}=\frac{2 \cdot \mathrm{TP}}{2 \cdot \mathrm{TP}+\mathrm{FP}+\mathrm{FN}} \\
\text { where, TP: true positive, FP: false positive, FN: false negative. }\end{array}$}} \\
\hline ASM: & & \\
\hline DSC: & & \\
\hline GD: & \multicolumn{2}{|l|}{ Gravity distance. } \\
\hline HD: & \multicolumn{2}{|l|}{ Hausdorff distance. } \\
\hline MD: & \multicolumn{2}{|l|}{ Mean distance. } \\
\hline MAD: & \multicolumn{2}{|l|}{ Mean absolute distance. } \\
\hline N/A: & \multicolumn{2}{|l|}{ Not applicable. } \\
\hline OR: & \multicolumn{2}{|l|}{ Overlap ratio. } \\
\hline OAE: & \multicolumn{2}{|c|}{ Overlapping area error; $\mathrm{OAE}=\frac{\mathrm{FP}+\mathrm{FN}}{\mathrm{TP}+\mathrm{FN}} \%$} \\
\hline PPV: & \multicolumn{2}{|c|}{ Positive predictive value; $\mathrm{PPV}=\frac{\mathrm{TP}}{\mathrm{TP}+\mathrm{PP}}$. } \\
\hline RMSD: & \multicolumn{2}{|l|}{ Root mean squared distance. } \\
\hline RVM: & \multicolumn{2}{|l|}{ Relevance vector machine. } \\
\hline SEN: & \multicolumn{2}{|l|}{ Sensitivity; $\mathrm{SEN}=\frac{\mathrm{TP}}{\mathrm{TP}+\mathrm{FN}}$. } \\
\hline SPE: & \multicolumn{2}{|c|}{ Specificity; $\mathrm{SPE}=\frac{\mathrm{TN}}{\mathrm{TN}+\mathrm{FP}} ;$ where $\mathrm{TN}$ : true negative. } \\
\hline VPC: & \multicolumn{2}{|l|}{ Volume properly contoured. } \\
\hline
\end{tabular}


TABLE 3: Summary of MRI-based CAD systems for prostate cancer detection and diagnosis, including segmentation method, selected features, classifier, and experimental performance.

\begin{tabular}{|c|c|c|c|}
\hline Study & Technique & Features & Performance \\
\hline $\begin{array}{l}\text { Chan et } \\
\text { al. [66] }\end{array}$ & $\begin{array}{l}\text { T2WI,DWI, } \\
\text { T2 map }\end{array}$ & $\begin{array}{l}\text { - Manual ROIs } \\
\text { - Gabor filter } \\
\text { - SVM }\end{array}$ & $\begin{array}{l}\text { - } 5 \text { Subjects (33 im- } \\
\text { ages) } \\
\text { - } A_{z}: 0.83 \pm 0.064\end{array}$ \\
\hline $\begin{array}{l}\text { Litjens et } \\
\text { al. [78] }\end{array}$ & $\begin{array}{l}\text { DWI, } \\
\text { DCE-MRI, } \\
\text { T2WI }\end{array}$ & 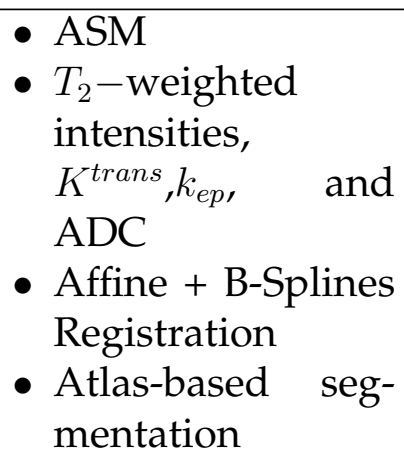 & $\begin{array}{l}\text { - } 188 \text { subjects } \\
\text { - SEN: } 0.747 \text { (at } 7 \mathrm{FP} \\
\text { per patient) and } \\
0.834 \text { (at } 9 \mathrm{FP} \text { per } \\
\text { patient) }\end{array}$ \\
\hline $\begin{array}{l}\text { Engelbrecht } \\
\text { et al. [68] }\end{array}$ & DCE-MRI & $\begin{array}{l}\text { - Manual ROIs } \\
\text { - time to peak, peak } \\
\text { enhancement, } \\
\text { washout } \\
\text { - N/A }\end{array}$ & $\begin{array}{l}\text { - } 36 \text { subjects } \\
\text { - } A_{z} \text { PZ: } 0.79 \\
\text { - } A_{z} \text { CZ: } 0.70\end{array}$ \\
\hline $\begin{array}{l}\text { Tiwari et } \\
\text { al. [80] }\end{array}$ & $\begin{array}{l}\text { T2WI, } \\
\text { MRS }\end{array}$ & $\begin{array}{l}\text { - Manual ROIs } \\
\text { - Random Forest } \\
\text { - N/A }\end{array}$ & $\begin{array}{l}\text { - } 36 \text { subjects } \\
\text { - } A U C=0.79 \pm 0.02\end{array}$ \\
\hline $\begin{array}{l}\text { Rouvière et } \\
\text { al. [70] }\end{array}$ & $\begin{array}{l}\text { DCE- } \\
\text { MRI,T2WI }\end{array}$ & $\begin{array}{l}\text { - Manual ROIs } \\
\text { - } \mathrm{N} / \mathrm{A} \\
\text { - Evaluation and } \\
\text { scoring by three } \\
\text { independent } \\
\text { readers }\end{array}$ & $\begin{array}{l}\text { - } 22 \text { subjects } \\
\text { - ACC: } 0.59 \quad\left(T_{2}-\right. \\
\text { weighted MRI) } \\
\text { - ACC: } 0.75 \text { (DCE- } \\
\text { MRI) }\end{array}$ \\
\hline $\begin{array}{l}\text { Kim et } \\
\text { al. [27] }\end{array}$ & $\begin{array}{l}\text { DCE- } \\
\text { MRI,T2WI }\end{array}$ & $\begin{array}{l}\text { - Manual ROIs } \\
\text { - Wash-in rate } \\
\text { - A cut-off thresh- } \\
\text { old selected by } \\
\text { a radiologist dif- } \\
\text { ferentiate cancer } \\
\text { from normal } \\
\text { tissue }\end{array}$ & $\begin{array}{l}\text { - } 53 \text { subjects } \\
\text { - ACC: } 0.62 \quad\left(T_{2}-\right. \\
\text { weighted MRI) } \\
\text { - ACC: } 0.88 \text { (DCE- } \\
\text { MRI) }\end{array}$ \\
\hline $\begin{array}{l}\text { Madabhushi } \\
\text { et al. [67] }\end{array}$ & $\begin{array}{l}\text { DCE- } \\
\text { MRI,T2WI,I }\end{array}$ & $\begin{array}{l}\text { - N/A } \\
\text { - Gabor filter } \\
\text { - Bayes classifier }\end{array}$ & $\begin{array}{l}\text { - } 5 \text { data set (33 } \\
\text { slices) } \\
\text { - SEN: 0.42, SPE: } \\
0.97 \text { and PPV: } 0.43\end{array}$ \\
\hline \multicolumn{4}{|c|}{ continued on the next page ... } \\
\hline
\end{tabular}


TABLE 3 - continued from the previous page

\begin{tabular}{|c|c|c|c|}
\hline Study & Technique & Features & Performance \\
\hline $\begin{array}{l}\text { Madabhushi } \\
\text { et al. [81] }\end{array}$ & $\begin{array}{l}\text { DCE- } \\
\text { MRI,T2WI }\end{array}$ & $\begin{array}{l}\text { - } \mathrm{N} / \mathrm{A} \\
\text { - Gabor filter } \\
\text { - Bayes and kNN- } \\
\text { nearest neighbor } \\
\text { classifiers }\end{array}$ & $\begin{array}{l}\text { - } 5 \text { data set }(33 \\
\text { slices) } \\
\text { - } A_{z}: 0.957\end{array}$ \\
\hline $\begin{array}{l}\text { Fütterer et } \\
\text { al. [69] }\end{array}$ & DCE-MRI & $\begin{array}{l}\text { - Manual ROIs } \\
\text { - wash-out slopes } \\
\text { - Prospectively } \\
\text { evaluation and } \\
\text { scoring of the se- } \\
\text { lected features by } \\
\text { two independent } \\
\text { radiologists }\end{array}$ & $\begin{array}{l}\text { - } 34 \text { subjects } \\
\text { - } A_{z}: 0.68\end{array}$ \\
\hline $\begin{array}{l}\text { Viswanath } \\
\text { et al. [73] }\end{array}$ & DCE-MRI & $\begin{array}{l}\text { - ASM } \\
\text { - Ratio of the wash- } \\
\text { in and wash-out } \\
\text { rates } \\
\text { - K-means cluster- } \\
\text { ing }\end{array}$ & $\begin{array}{l}\text { - } 21 \text { subjects } \\
\text { - SEN: } 60.72 \% \text {, SPE: } \\
\text { 83.24\%, and ACC: } \\
77.2 \%\end{array}$ \\
\hline $\begin{array}{l}\text { Ocak et al. } \\
\text { [72] }\end{array}$ & $\begin{array}{l}\text { T2WI, } \\
\text { DCE-MRI }\end{array}$ & $\begin{array}{l}\text { - Manual ROIs } \\
\text { - the area under } \\
\text { the gadolinium } \\
\text { concentration } \\
\text { curve } \\
\text { - Logistic regression } \\
\text { modeling }\end{array}$ & 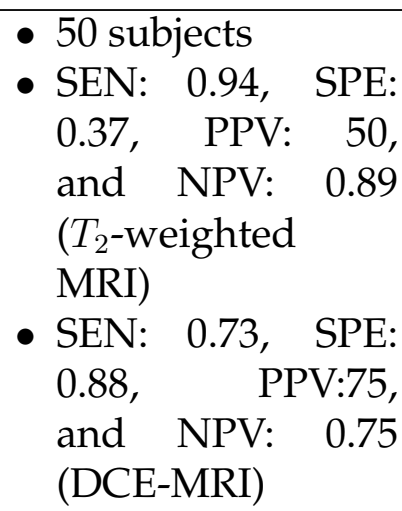 \\
\hline $\begin{array}{l}\text { Puech et al. } \\
\text { [71] }\end{array}$ & DCE-MRI & $\begin{array}{l}\text { - Manual ROIs } \\
\text { - Wash-in and } \\
\text { wash-out slopes } \\
\text { - Automatic scoring } \\
\text { algorithm }\end{array}$ & $\begin{array}{l}\text { - } 84 \text { subjects } \\
\text { - for PZ SEN: } 100 \% \\
\text { and SPE: } 45 \% \\
\text { - for TZ SEN: } 100 \% \\
\text { and SPE: } 40 \%\end{array}$ \\
\hline $\begin{array}{ll}\text { Vos } & \text { et } \\
\text { al. [76] } & \end{array}$ & $\begin{array}{l}\text { DCE-MRI, } \\
\text { T2WI }\end{array}$ & $\begin{array}{l}\text { - Manual ROIs } \\
\text { - Quantitative } \\
\text { features were } \\
\text { extracted from a } \\
\text { kinetic modelling } \\
\text { of the enhance- } \\
\text { ment curve } \\
\text { - SVM }\end{array}$ & $\begin{array}{l}\text { - } 34 \text { subjects } \\
\text { - } A_{z}: 0.83\end{array}$ \\
\hline
\end{tabular}


TABLE 3 - continued from the previous page

\begin{tabular}{|c|c|c|c|}
\hline Study & Technique & Features & Performance \\
\hline $\begin{array}{l}\text { Ampeliotis } \\
\text { et al. [77] }\end{array}$ & $\begin{array}{l}\text { T2WI, } \\
\text { DCE-MRI }\end{array}$ & $\begin{array}{l}\text { - Manual ROIs } \\
\text { - } T_{2} \text {-weighted fea- } \\
\text { tures (intensity, } \\
\text { and the intensity } \\
\text { variance) } \\
\text { - Probabilistic neu- } \\
\text { ral networks }\end{array}$ & $\begin{array}{l}\text { - } 10 \text { subjects } \\
\text { - } A_{z}: 0.89 \quad\left(T_{2}-\right. \\
\text { weighted MRI) } \\
\text { and (DCE-MRI) }\end{array}$ \\
\hline $\begin{array}{l}\text { Viswanath } \\
\text { et al. [82] }\end{array}$ & DCE-MRI & $\begin{array}{l}\text { - ASM } \\
\text { - } T_{2} \text { features (stan- } \\
\text { dard deviation, } \\
\text { gradient, Haralick } \\
\text { feature), and DCE } \\
\text { features (washin } \\
\text { and wash-out) } \\
\text { - Bayes classifier } \\
\text { and random forest }\end{array}$ & $\begin{array}{l}\text { - } 6 \text { subjects (18 Im- } \\
\text { ages) } \\
\text { - } A_{z}: 0.815 \pm 0.029\end{array}$ \\
\hline $\begin{array}{l}\text { Lopes et al. } \\
{[83]}\end{array}$ & $\overline{D W I}$ & $\begin{array}{l}\text { - Manual ROIs } \\
\text { - Fractal dimen- } \\
\text { sion, and Multi- } \\
\text { fractional Brown- } \\
\text { ian motion } \\
\text { - SVM and Ad- } \\
\text { aBoost }\end{array}$ & $\begin{array}{l}\text { - } 17 \text { subjects } \\
\text { - SEN: } 0.83 \text { and SPE: } \\
0.91 \text { for AdaBoost } \\
\text { - SEN: } 0.85 \text { and SPE: } \\
0.93 \text { for SVM }\end{array}$ \\
\hline $\begin{array}{l}\text { Shah et al. } \\
\text { [84] }\end{array}$ & $\begin{array}{l}\text { T2WI, } \\
\text { DWI, } \\
\text { DCE-MRI }\end{array}$ & $\begin{array}{l}\text { - K-means } \\
\text { - } T_{2} \text { intensities } \\
\text { and Quantitative } \\
\text { features were } \\
\text { extracted from a } \\
\text { kinetic modelling } \\
\text { of the enhance- } \\
\text { ment curve and } \\
\text { ADC } \\
\text { - SVM }\end{array}$ & $\begin{array}{l}\text { - } 24 \text { subjects } \\
\text { - F-M: 0.93, Raters } \\
\text { agreement } \\
\text { (Kappa): } 0.89\end{array}$ \\
\hline \multicolumn{4}{|c|}{ continued on the next page ... } \\
\hline
\end{tabular}


TABLE 3 - continued from the previous page

\begin{tabular}{|c|c|c|c|}
\hline Study & Technique & Features & Performance \\
\hline $\begin{array}{l}\text { Sung et al. } \\
{[85]}\end{array}$ & DCE-MRI & $\begin{array}{l}\text { - Manual ROIs } \\
\text { - } T_{2} \text { baseline and } \\
\text { peak signal in- } \\
\text { tensities, initial } \\
\text { up-slope, wash- } \\
\text { in and washout } \\
\text { rates, time to peak, } \\
\text { percentage of rela- } \\
\text { tive enhancement, } \\
\text { percentage en- } \\
\text { hancement ratio, } \\
\text { time of arrival } \\
\text { SVM }\end{array}$ & $\begin{array}{l}\text { - } 42 \text { subjects } \\
\text { - SEN: 0.77, SPE: } \\
0.77 \text {, and ACC: } \\
0.83\end{array}$ \\
\hline \begin{tabular}{ll|} 
Vos & et \\
al. [75] &
\end{tabular} & $\begin{array}{l}\text { DWI, } \\
\text { DCE-MRI }\end{array}$ & $\begin{array}{l}\text { - ASM } \\
\text { - } T_{2} \text { intensities } \\
\text { and Quantitative } \\
\text { features were } \\
\text { extracted from a } \\
\text { kinetic modelling } \\
\text { of the enhance- } \\
\text { ment curve and } \\
\text { ADC } \\
\text { - LDA }\end{array}$ & $\begin{array}{l}\text { - } 200 \text { subjects } \\
\text { - SEN: 0.41 (at } 1 \\
\text { FPs), } 0.65 \text { (at } 3 \\
\text { FPs), and } 0.74 \text { ( at } \\
5 \text { FPs) }\end{array}$ \\
\hline $\begin{array}{l}\text { Niaf et al. } \\
{[86]}\end{array}$ & $\begin{array}{l}\text { T2WI, } \\
\text { DCE-MRI, } \\
\text { DWI }\end{array}$ & $\begin{array}{l}\text { - Manual ROIs } \\
\text { - } T_{2} \text { Quantitative } \\
\text { features were } \\
\text { extracted from a } \\
\text { kinetic modelling } \\
\text { of the enhance- } \\
\text { ment curve } \\
\text { - Nonlinear SVM, } \\
\text { LDA, kNN- } \\
\text { nearest neighbor, } \\
\text { and naïve Bayes } \\
\text { classifiers }\end{array}$ & $\begin{array}{l}\text { - } 30 \text { subjects } \\
\text { - } A_{z}: 0.89\end{array}$ \\
\hline $\begin{array}{l}\text { Peng et al. } \\
\text { [79] }\end{array}$ & $\begin{array}{l}\text { T2WI, } \\
\text { DCE-MRI, } \\
\text { DWI }\end{array}$ & $\begin{array}{l}\text { - Manual ROIs } \\
\text { - } T_{2} \text { Quantitative } \\
\text { features were } \\
\text { extracted from a } \\
\text { kinetic modelling } \\
\text { and ADC } \\
\text { - LDA }\end{array}$ & $\begin{array}{l}\text { - } 48 \text { subjects } \\
\text { - } A_{z}: 0.95 \pm 0.02\end{array}$ \\
\hline $\begin{array}{l}\text { AMM: } \\
\text { ASM: }\end{array}$ & \multicolumn{3}{|c|}{$\begin{array}{l}\text { Active appearance model. } \\
\text { Active shape model. }\end{array}$} \\
\hline
\end{tabular}


TABLE 3 - continued from the previous page

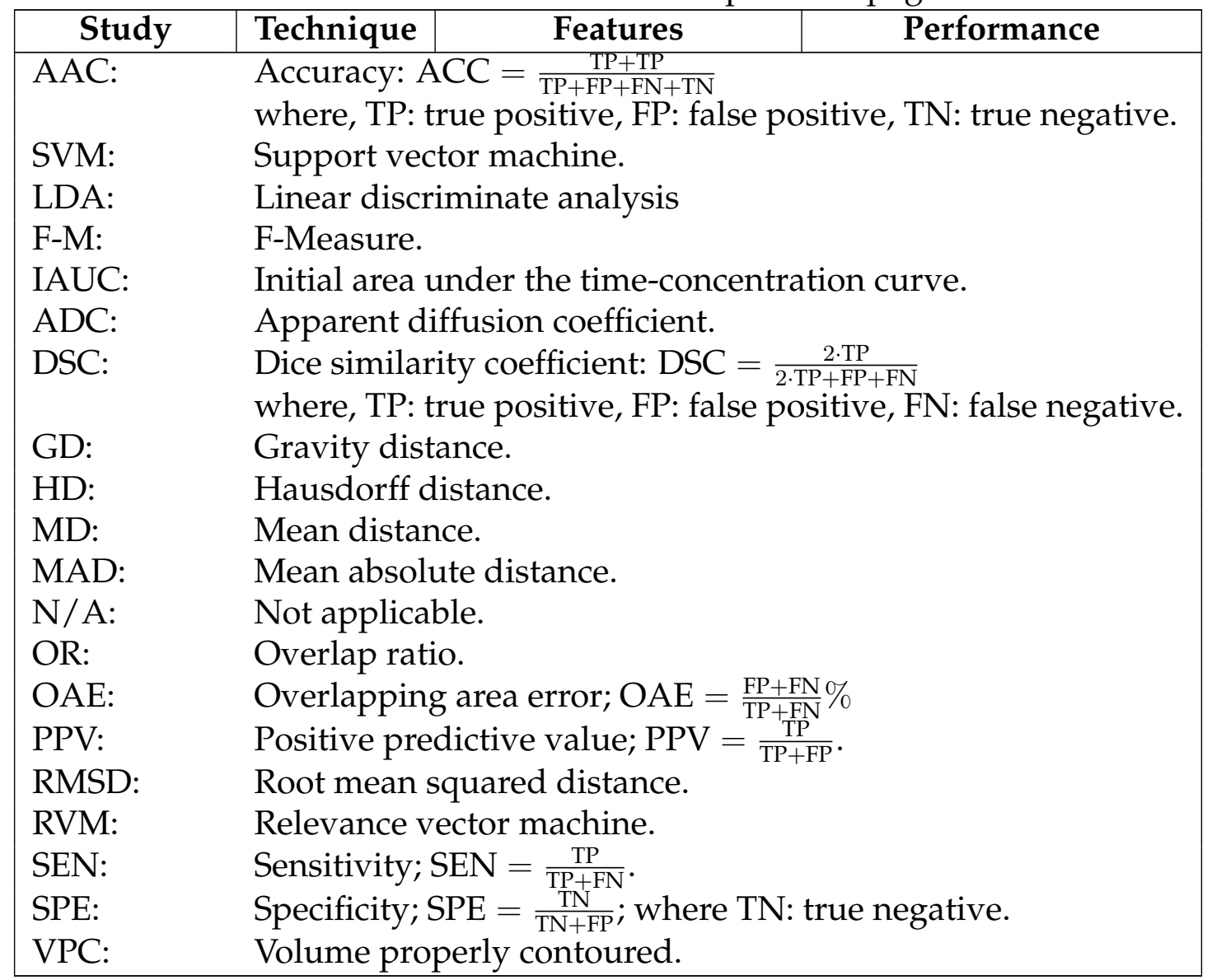

In summary, developing noninvasive CAD systems for the detection and diagnosis of prostate cancer is an area of research interest. Current CAD systems focus mainly on the initial voxel classification stage by obtaining likelihood maps that combine information from MR images using mathematical descriptors. State-of-the art studies showed that voxel basis discrimination between benign and malignant tissue is feasible with good performances. However, the majority of these studies were performed by radiologists who selected an ROI (small window) around the prostate and followed signal changes within these ROIs. In addition, the final diagnosis and patient management is left to the radiologist. Unfortunately, such approaches not only require manual interaction of the operators, but also ROI selection biases the final decision and brings up the same issue of over- or under-estimating the problem in the entire gland, just as with biopsy. 
Moreover, manual ROI selection and function curve generation from these ROIs assume that the prostates (prostate contours) remain exactly the same from scan to scan. Nonetheless, prostate contours may not always exactly match due to patient movement or breathing effects; therefore, motion correction techniques should be applied first before ROI selection. Also, to automate the algorithm and to cancel ROI dependency, segmentation approaches that can separate the prostate from the surrounding structures are needed. 


\section{CHAPTER IV}

\section{A NOVEL IMAGE-BASED APPROACH FOR EARLY DETECTION OF PROSTATE CANCER USING DCE-MRI}

This chapter presents a novel non-invasive approach for early diagnosis of prostate cancer from dynamic contrast enhanced magnetic resonance imaging (DCE-MRI). In order to precisely analyze the complex 3D DCE-MRI of the prostate, a novel processing frame work that consists of four main steps is proposed. The first step is to isolate the prostate from the surrounding anatomical structures based on a Maximum a Posteriori (MAP) estimate of a log-likelihood function that accounts for the shape priori, the spatial interaction, and the current appearance of the prostate tissues and its background (surrounding anatomical structures). In the second step, a non-rigid registration algorithm is employed to account for any local deformation that could occur in the prostate during the scanning process due to the patient's breathing and local motion. In the third step, the perfusion curves that show propagation of the contrast agent into the tissue are obtained from the segmented prostate of the whole image sequence of the patient. In the final step, we collect two features from these curves and use a $k_{n}$-Nearest Neighbor classifier classifier to distinguish between malignant and benign detected tumors. Moreover, in this chapter, we introduce a new approach to generate color maps that illustrate the propagation of the contrast agent in the prostate tissues based on the analysis of the 3D spatial interaction of the change of the gray level values of prostate voxels using a Generalized Gauss-Markov Random Field (GGMRF) image model. Finally, the tumor boundaries are determined using a level set de- 
formable model controlled by the perfusion information and the spatial interactions between the prostate voxels. Experimental results on 30 clinical DCE-MRI data sets yield promising results.

\section{A. INTRODUCTION}

Neoangiogenesis is the key component of the development of prostate cancer, composed of increases in both the density and permeability of blood vessels within the cancerous area $[87,88]$. These microvascular alterations result in differences in the enhancement pattern of prostate cancer compared with benign prostate tissue when performing Dynamic Contrast-Enhanced MRI (DCE-MRI). These differences are multifactorial and include earlier and more intense enhancement as well as more rapid washout of contrast material within the tumor [89]. Studies have shown dynamic contrast enhancement to improve the performance of MRI for the localization of prostate cancer, raising the sensitivity for tumor in comparison with either $T_{2}$-weighted imaging alone. This in turn contributes to a role for DCE-MRI in directing prostate biopsy [88] as well as in preoperative staging and surgical planning $[69,90]$. DCE-MRI poses multiple challenges stemming from the need to image very quickly, to capture the transient first-pass transit event, while maintaining adequate spatial resolution. Other factors such as varying signal intensities over the time course of agent transit also complicate segmentation procedures. Nonrigid deformations, or shape changes, may occur related to pulsatile or transmitted effects from adjacent structures, such as bowel.

Most prostate CAD researchers [27,68-71,76] have focused on the initial voxel classification stage. They obtained likelihood maps by combining information from MR images using mathematical descriptors. These studies showed on a voxel basis that the discrimination between benign and malignant tissue is fea- 
sible with good performances. However, these studies require user interaction to select a ROI around the prostate. In addition to the localization of the ROI of the tumor, the final diagnosis and patient management is left to the radiologist. However, the majority of these studies were performed by radiologists who selected a region of interest (ROI) (a small window) around the prostate and followed signal change within this region of interest. Unfortunately, such approaches not only require manual interaction of the operators, but also ROI selection biases the final decision and brings up the same issue of over or underestimating the problem in the entire graft, just as with biopsy. Moreover, manual window selection and generating a function curve from this window over a time-sequence of images, assumes that the prostates (prostate contours) remain exactly the same from scan to scan. However, prostate contours may not always exactly match due to patient movement or breathing effects; therefore, image registration schemes should be applied first before ROI selection. Also, to automate the algorithm and to cancel ROI dependency, segmentation algorithms that can separate the prostate from the surrounding structures are needed. To overcome these limitations, we propose an automatic framework for the early diagnosis of prostate cancer using DCE-MRI.

The proposed framework segments the prostate from the surrounding anatomical structures based on a MAP estimate of a new likelihood function. To handle the object inhomogeneities and variability and overcome image noise, the proposed likelihood function accounts for the visual appearances of the prostate and background, 3D spatial interaction between the prostate voxels, and a learned 3D shape model. Second, in order to account for any local prostate deformations that could occur during the scanning process, a nonrigid registration algorithm is employed, which is based on deforming a target prostate object over evolving isocontours to match a reference object. The correspondences between the target and reference objects are found by the solution of the Laplace equation as described in Section IV.B.2 In the third step, agent kinetic curves that show the contrast agent 
propagation into the tissue are obtained, and then used to collect two features to distinguish between malignant and benign detected tumors using a $k_{n}$-nearest neighbor classifier. Finally, parametric map displays that illustrate the propagation of the contrast agent into the prostate tissue are constructed for visual assessment and characterization of the physiological data. Details of the proposed framework are described in the following sections.

\section{B. Methods}

In this chapter we introduce a new, automated, and noninvasive framework for early diagnosis of prostate cancer by analyzing 3D DCE-MRI time series perfusion data is proposed see Figure 17. In the proposed DCE-MRI based framework, the classification of prostate cancer is performed using the following five steps:

1. Segmentation of the prostate objects from the surrounding abdominal structures on the DCE-MR images.

2. Nonrigid registration for local motion correction.

3. Distinguish between malignant and benign detected tumors.

The overall framework has been tested on a total of 30 dynamic DCE-MRI perfusion data sets to permit us to draw statistically meaningful inferences

1. Segmentation of the Prostate Using a Joint MGRF Model

The segmentation of the prostate is a challenge, since the gray-level distribution of the prostate and surrounding organs is not highly distinguishable and because of the anatomical complexity of prostate. This stage proposes a power- 


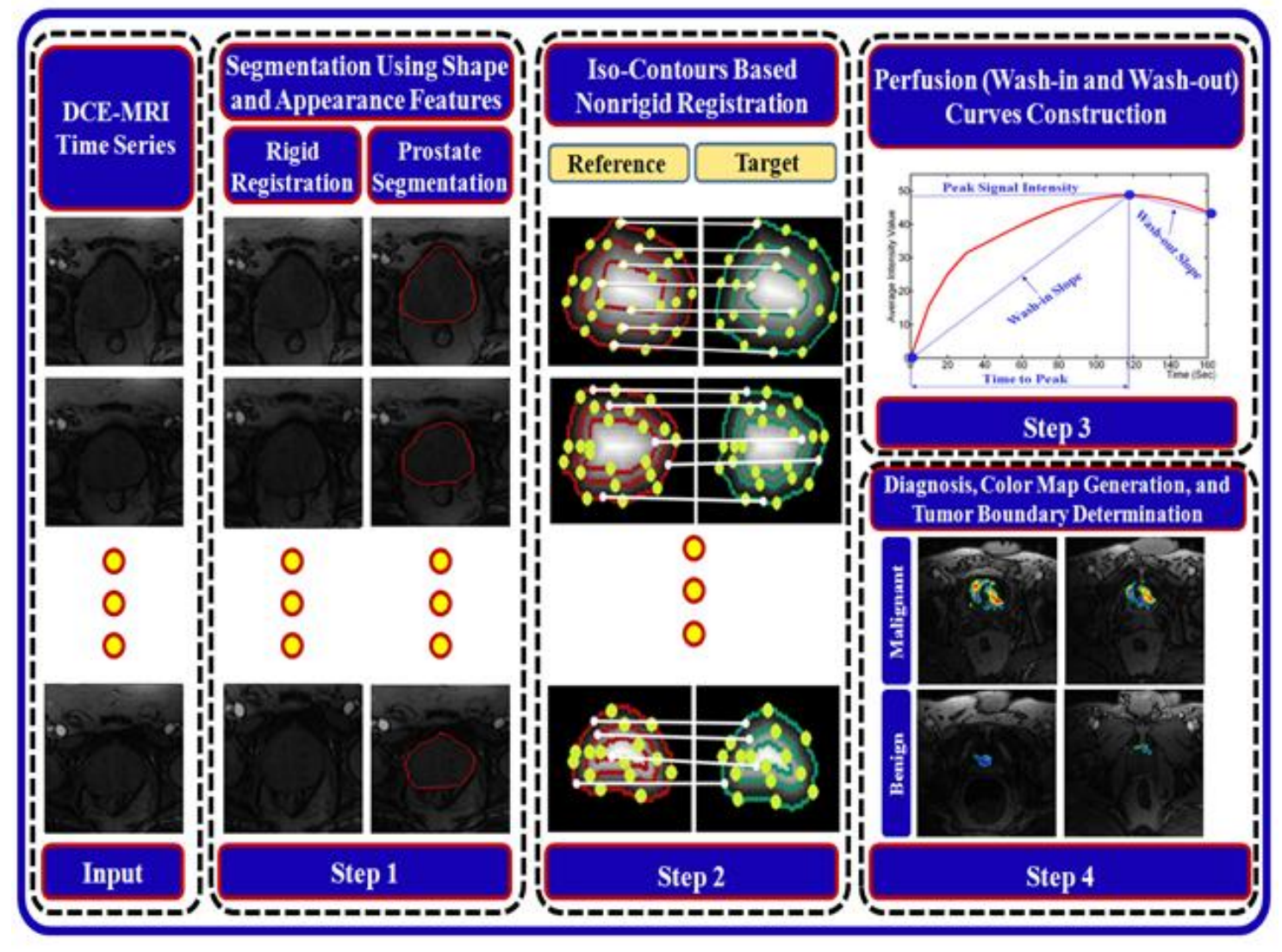

FIGURE 17: The proposed CAD system for early detection of prostate cancer

ful framework for prostate segmentation based on a learned shape model and an identifiable joint Markov-Gibbs Random Field (MGRF) model of DCE-MRI and "object-background" region maps. The joint-MGRF model is fundamentally a model that relates the joint probability of an image and its object-background region map, to geometric structure and to the energy of repeated patterns within the image. The basic theory behind such models is that they assume that the signals associated with each pixel depend on the signals of the neighboring pixels, and thus explicitly take into account their spatial interactions, and other features, such as shape.

Let $\mathbf{Q}=\{0, \ldots, Q-1\}, \mathbf{L}=\{\mathrm{ob}, \mathrm{bg}\}$, and $\mathbf{U}=[0,1]$ be a set of $Q$ integer gray levels, a set of object ("ob") and background ("bg") labels, and a unit interval, respectively. Let a $3 \mathrm{D}$ arithmetic grid $\mathbf{R}=\{(x, y, z): x=0,1, \ldots, X-1 ; y=$ 
$0,1, \ldots, Y-1 ; z=0,1, \ldots, Z-1\}$ support grayscale DCE-MRI $\mathbf{g}: \mathbf{R} \rightarrow \mathbf{Q}$ and their binary region maps $\mathbf{m}: \mathbf{R} \rightarrow \mathbf{L}$, and probabilistic shape model $\mathbf{s}: \mathbf{R} \rightarrow \mathbf{U}$. The shape model allows for registering (aligning) 3D prostate DCE-MRI. The DCEMR data $\mathbf{g}$ and its region maps $\mathbf{m}$ are described with a joint probability model:

$$
P(\mathbf{g}, \mathbf{m})=P(\mathbf{g} \mid \mathbf{m}) P_{\mathrm{h}}(\mathbf{m})
$$

where $P(\mathbf{g} \mid \mathbf{m})$ is a conditional distribution of the images given the map and $P_{\mathrm{h}}(\mathbf{m})=P_{\mathrm{s}}(\mathbf{m}) P(\mathbf{m})$ is an unconditional probability distribution of maps. The map model $P_{\mathrm{h}}(\mathbf{m})=P_{\mathrm{s}}(\mathbf{m}) P(\mathbf{m})$ has two independent parts: a shape prior being a spatially variant independent random field of region labels $P_{\mathrm{s}}(\mathbf{m})$, for a set of co-aligned training DCE-MR data, and a $2^{\text {nd }}$-order MGRF model $P(\mathbf{m})$ of spatially homogeneous evolving map $\mathbf{m}$.

The Bayesian MAP estimate of the map, given the MR data $\mathrm{g}$, $\mathbf{m}^{*}=\arg \max _{\mathbf{m}} L(\mathbf{g}, \mathbf{m})$ maximize the log-likelihood function:

$$
L(\mathbf{g}, \mathbf{m})=\log (P(\mathbf{g} \mid \mathbf{m}))+\log \left(P_{\mathrm{h}}(\mathbf{m})\right)
$$

In this work we focus on accurate identification of the spatial interaction between the prostate voxels $\left(P_{\mathrm{h}}(\mathbf{m})\right.$, and the intensity distribution for the prostate tissues, $(P(\mathbf{g} \mid \mathbf{m}))$, as shown in Figure.18. 


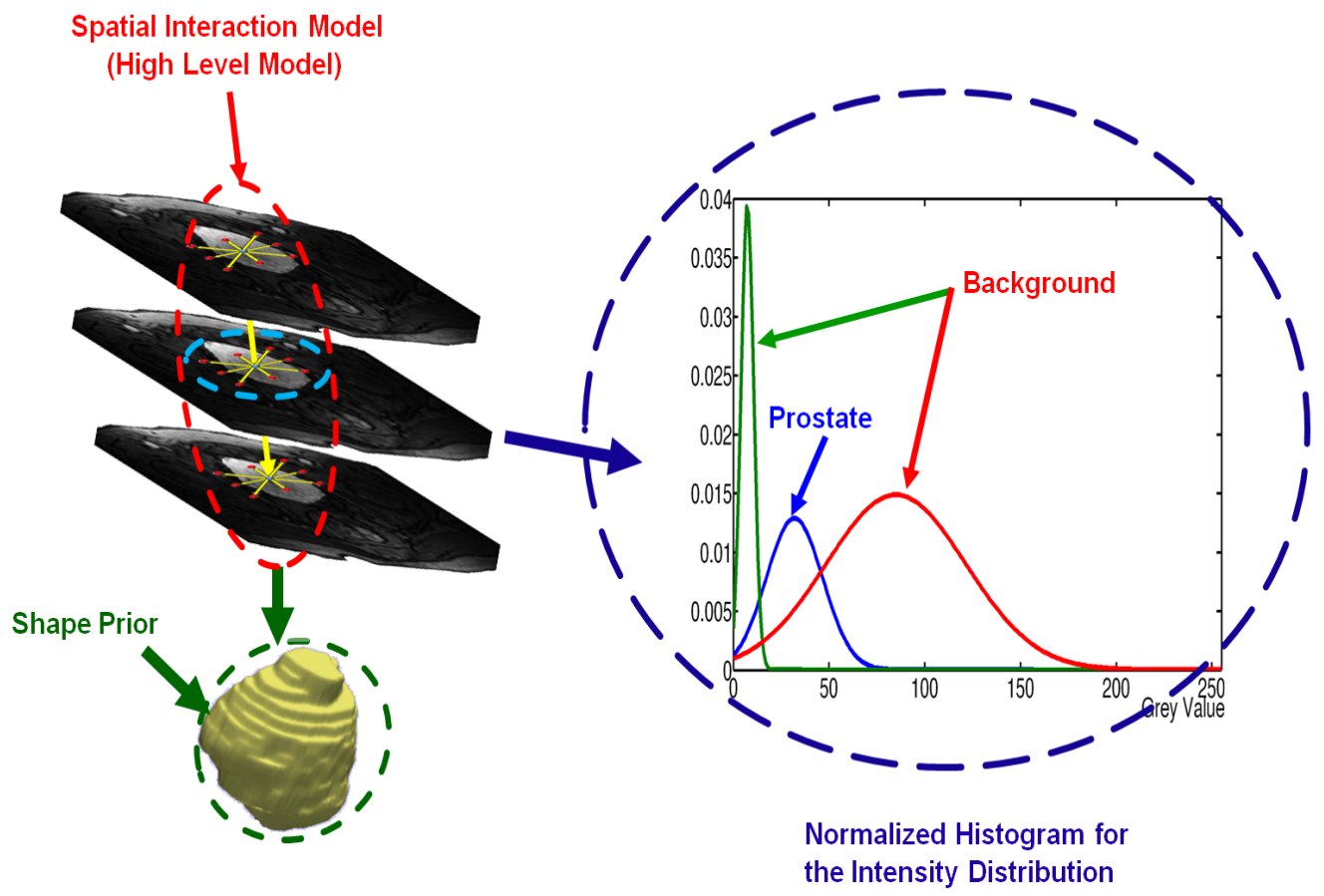

FIGURE 18: Illustration of the Joint Markov-Gibbs random field (MGRF) image model of the prostate DCE-MRI. 
To perform the initial prostate segmentation, a given 3D DCE-MR images is aligned to one of the training 3D DCE-MR images. The shape model provides the voxels-wise object and background probabilities being used, together with the conditional image intensity model $P(\mathbf{g} \mid \mathbf{m})$, to build an initial region map. The final Bayesian segmentation is performed using the identified joint MGRF model of the DCE-MRI and region maps.

\section{a. Conditional Intensity Model The specific visual appearance of the} prostate in each data set to be segmented is taken into account by modeling a marginal gray level distribution with a linear combination of discrete Gaussians (LCDG) [91-93]. Unlike the conventional modeling with a mixture of Gaussians [94] or other simple distributions [95], one per dominant mode, we accurately approximate the empirical signal distributions with a linear combination of sign-alternate discrete Gaussians, and partition the LCDG for the whole image into sub-models relating to each dominant mode, i.e. the prostate and the background.

A discrete Gaussian (DG) $\boldsymbol{\Psi}_{\theta}=(\psi(q \mid \theta): q \in \mathbf{Q})$ is defined as a discrete probability distribution with $Q$ components obtained by integrating a continuous 1-D Gaussian density $\varphi(q \mid \theta)$ with parameters $\theta=(\mu, \sigma)$, where $\mu$ is the mean and $\sigma^{2}$ is the variance, over $Q$ intervals related to the successive signal values in Q: if $\Phi_{\theta}(q)=\int_{-\infty}^{q} \varphi(z \mid \theta) d z$ is the cumulative Gaussian probability function, then $\psi(0 \mid \theta)=\Phi_{\theta}(0.5), \psi(q \mid \theta)=\Phi_{\theta}(q+0.5)-\Phi_{\theta}(q-0.5)$ for $q=1, \ldots, Q-2$, and $\psi(Q-1 \mid \theta)=1-\Phi_{\theta}(Q-1.5)$. The LCDG, $\mathbf{P}_{\mathbf{w}, \boldsymbol{\Theta}}=\left[p_{\mathbf{w}, \Theta}(q): q \in \mathbf{Q}\right]$; $\sum_{q \in \mathbf{Q}} p_{\mathbf{w}, \boldsymbol{\Theta}}(q)=1$, with two positive dominant and multiple positive, $C_{\mathrm{p}} \geq 2$, and negative, $C_{\mathrm{n}} \geq 0$, DGs subordinate is defined as follows [92]:

$$
p_{\mathbf{w}, \Theta}(q)=\sum_{k=1}^{C_{\mathrm{p}}} w_{\mathrm{p}: k} \psi\left(q \mid \theta_{\mathrm{p}: k}\right)-\sum_{l=1}^{C_{\mathrm{n}}} w_{\mathrm{n}: l} \psi\left(q \mid \theta_{\mathrm{n}: l}\right)
$$

with the non-negative weights $\mathbf{w}=\left[w_{\mathrm{p}: k}, w_{\mathrm{n}: l}\right]$ that meet the obvious constraint $\sum_{k=1}^{C_{\mathrm{p}}} w_{\mathrm{p}: k}-\sum_{l=1}^{C_{\mathrm{n}}} w_{\mathrm{n}: l}=1$. The subordinate DGs approximate closely the deviations of the empirical distribution from the conventional mixture of the dominant 
positive DGs.

Given the number $K$ of the dominant DGs (in our particular case $K=2$ ), the numbers $C_{\mathrm{p}}-K$ and $C_{\mathrm{n}}$ of the subordinate components, as well as the parameters $\mathrm{w}, \Theta$ (i.e., the weights, the means, and the variances) of all the DGs are estimated first with a sequential initializing expectation maximization (EM) based algorithm producing a close initial LCDG-approximation of the empirical distribution. Then, under the fixed numbers of the components, $C_{\mathrm{p}}$ and $C_{\mathrm{n}}$, all the other parameters are refined with an EM algorithm, modified to account for the signalternate components [96]. The refined LCDG model is finally partitioned into two LCDG sub-models, one per class, by associating the subordinate DGs with the dominant components in such a way that the misclassification rate is minimal. The LCDG of Equation 3, including the numbers $C_{p}$ and $C_{n}$ of its components, is identified using the expectation-maximization (EM)-based algorithm introduced in [97-103].

b. Spatial Voxel Interaction Model To overcome the noise effect and to ensure the homogeneity of the segmentation, spatial voxel interactions between the region labels are also taken into account using a generic MGRF of the region map. A generic MGRF of region maps $[96,104]$ accounts only for 2D pairwise interaction between each region label and its characteristic neighbors. Generally, the interaction structure and the Gibbs potentials can be arbitrary and are identified from the training data. For simplicity, we restrict the interaction structure to the nearest voxel 26- neighborhood only as shown in Figure. 19. By symmetry considerations, we assume that the potentials are independent of relative orientation of each voxel pair and depend only on intra- or inter-region position (i.e. whether the labels are equal or not). Under these restrictions, it is the 3D extension of the conventional auto-binomial, or Potts model differing only in that the potentials are estimated analytically. 


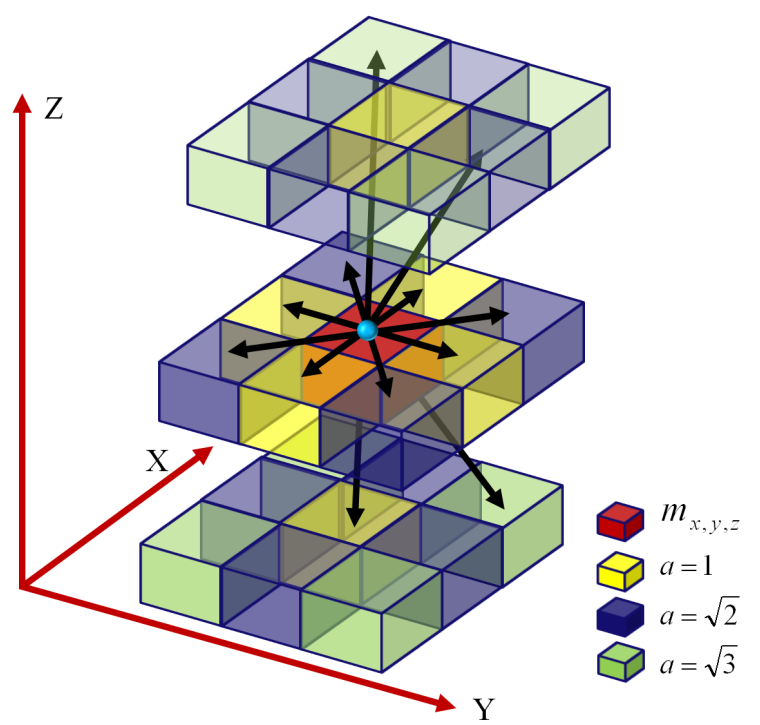

FIGURE 19: 3D $2^{\text {nd }}$ order MGRF neighborhood system. Note that the reference voxel is shown in red and $d$ represents the absolute distance between two voxels in the same and adjacent MRI slices, or cross-sections.

The 26-neighborhood has three types of symmetric pairwise interactions specified by the absolute distance $a$ between two voxels in the same and adjacent MRI slices ( $a=1, \sqrt{2}$, and $\sqrt{3}$, respectively): (i) the closest pairs with the inter-voxel coordinate offsets $\mathbf{N}_{1}=\{(1,0,0),(0,1,0),(0,0,1)\}$; (ii) the farther diagonal pairs with the offsets $\mathbf{N}_{\sqrt{2}}=\{(0,1, \pm 1),(1,0, \pm 1),(1, \pm 1,0)\}$, and (iii) the farthest diagonal pairs with the offsets $\mathbf{N}_{\sqrt{3}}=\{(1, \pm 1, \pm 1)\}$. The potentials of each type are bi-valued because only the coincidence of the labels is taken into account: $\mathbf{V}_{a}=\left\{V_{a, \text { eq }} ; V_{a, \text { ne }}\right\}$ where $V_{a, \text { eq }}=V_{a}\left(l, l^{\prime}\right)$ if $l=l^{\prime}$ and $V_{a, \text { ne }}=V_{a}\left(l, l^{\prime}\right)$ if $l \neq l^{\prime}$; $a \in \mathbf{A}=\{1, \sqrt{2}, \sqrt{3}\}$. Then the MGRF model of region maps is as follows:

$$
P(\mathbf{m})=\frac{1}{Z} \exp \sum_{(x, y, z) \in \mathbf{R}} \sum_{a \in \mathbf{A}} \sum_{(\xi, \eta, \kappa) \in \mathbf{N}_{a}} V_{a}\left(m_{x, y, z}, m_{x+\xi, y+\eta, z+\kappa}\right)
$$

where $Z$ is the normalizing factor (partition function). 
To identify the MGRF model described in the equation, approximate analytical maximum likelihood estimate of the 3D Gibbs potentials, $V_{a \text {, eq }}, V_{a, \text { ne }}$ are derived in line with $[91,96,105]$.

$$
V_{a, \mathrm{eq}}=-V_{a, \mathrm{ne}}=2\left(f_{a, \mathrm{eq}}(\mathbf{m})-\frac{1}{2}\right)
$$

where $f_{a \text {, eq }}(\mathbf{m})$ denotes the relative frequency of the equal label pairs in the equivalent voxel pairs $\{((x, y, z),(x+\xi, y+\eta, z+\kappa)):(x, y, z) \in \mathbf{R} ;(x+\xi, y+\eta, z+\kappa) \in \mathbf{R}$; $\left.(\xi, \eta, \kappa) \in \mathbf{N}_{a}\right\}$. of a region map $m$ of a given DCE-MRI aligned in accord with the prior shape model.

c. Probabilistic Shape Model To enhance the segmentation accuracy, additional constraints based on the expected shape of the prostate are introduced by co-aligning each given DCE-MR data to a training database and using a soft probabilistic 3D prostate shape model To circumvent this limitation, we use a soft probabilistic 3D prostate shape model $P_{\mathbf{s}}(\mathbf{m})=\prod_{(x, y, z) \in \mathbf{R}} S_{m_{x, y, z}}$; where $S_{m_{x, y, z}}$ is the empirical probability that the voxel $(x, y, z)$, belongs to the prostate $(\mathbf{L}=\mathrm{ob})$ or the background $(\mathbf{L}=b g$ ) given the map (see Figure.21). The soft template (see Figure.22) is constructed and updated as outlined in Algorithm IV.B.1.c and the proposed prostate segmentation process can be summarize as in Algorithm IV.B.1.c 

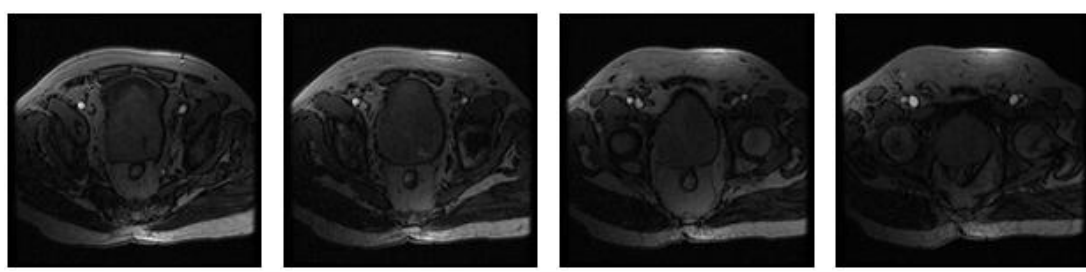

(a)
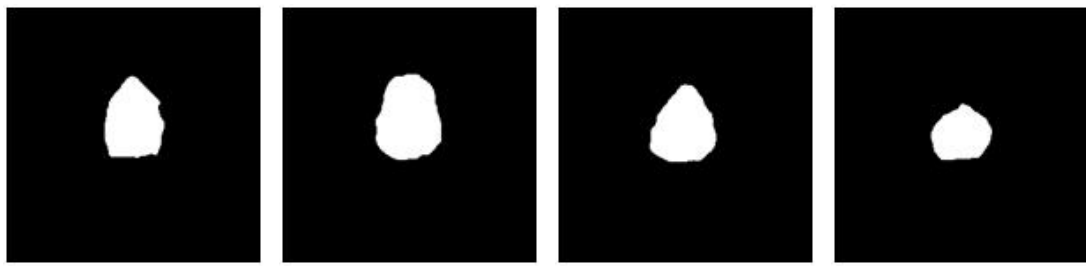

(b)
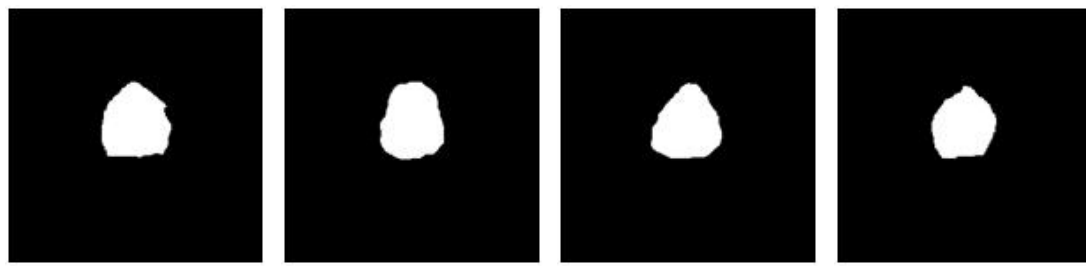

(c)

FIGURE 20: Forming the prostate shape prior onto 2D: $(a)$ training samples, $(b)$ manually segmented prostate regions, and $(b)$ their affine MI-based alignment. Note that the registration enhances the overlap between the prostate objects and thus reduce the variability of the final estimated shape.

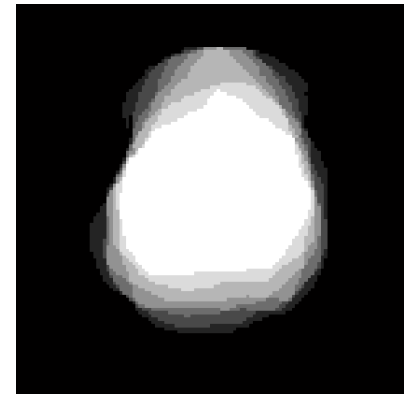

(a)

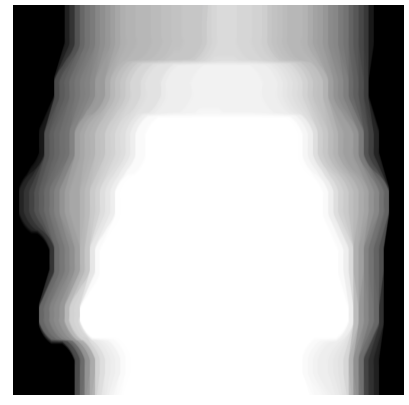

(b)

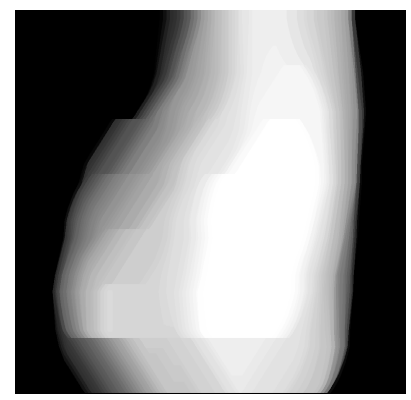

(c)

FIGURE 21: 3D prostate shape model projected onto 2D axial (a), saggital (b), and coronal (c) planes for visualization. 

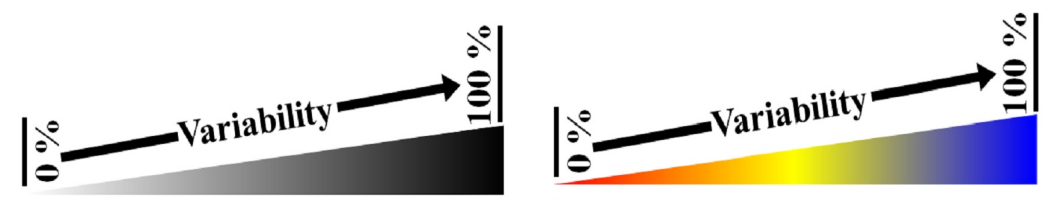

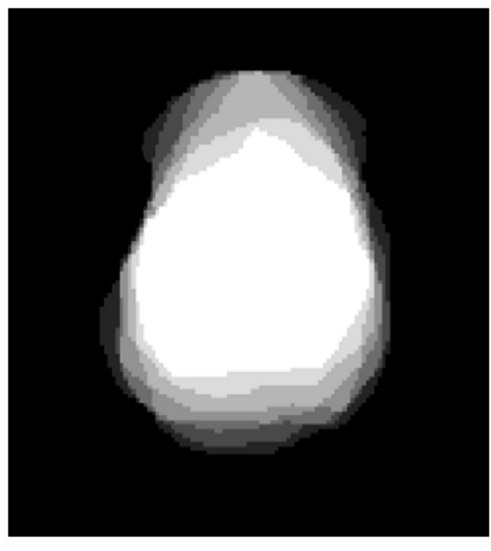

(a)

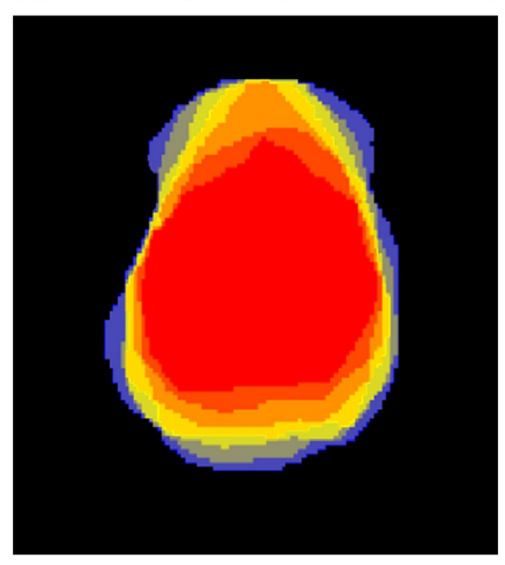

(b)

FIGURE 22: Gray-coded (a) and color-coded (b) axial view of the prostate shape prior. initial region map. The final Bayesian segmentation is performed using the identified joint MGRF model of the DCE-MRI data and its region maps. In total, the proposed prostate segmentation approach involves the steps summarized in Algorithm IV.B.1.c 


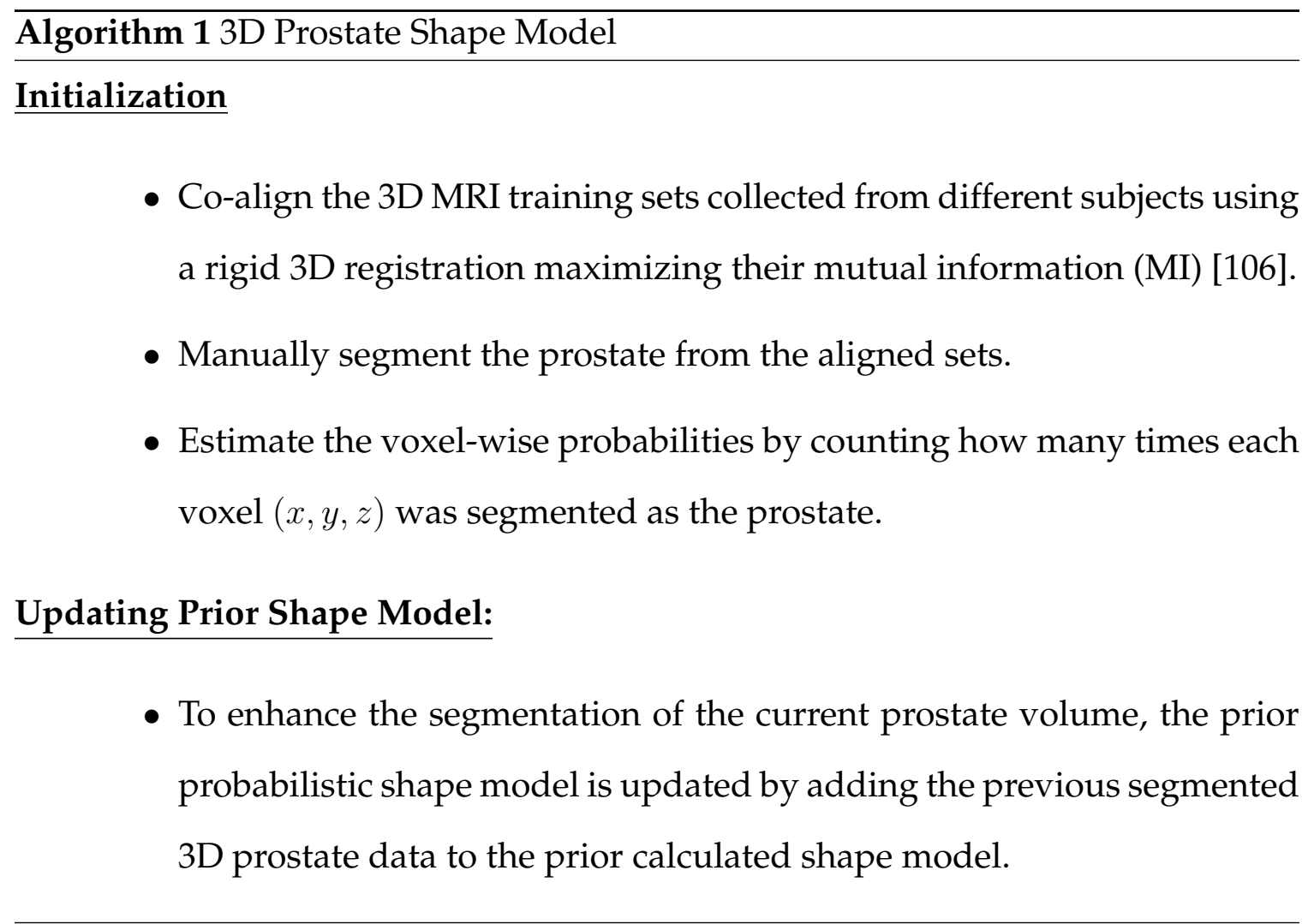


Algorithm 2 Prostate Segmentation Approach

Input: 3D DCE-MRI prostate data $g$ to be segmented.

Output: segmented prostate border (the final estimate $m$ ).

- Training Phase:

1. Co-align the 3D DCE-MRI training sets collected from different subjects using a rigid 3D registration maximizing their mutual information (MI) [106].

2. Manual delineation of the prostate borders from the co-aligned data

3. Estimate the voxel-wise probabilities by counting how many times each voxel $(x, y, z)$ was segmented as the prostate.

\section{- Testing Phase:}

1. Perform an affine alignment of a given 3D DCE-MRI to an arbitrary prototype prostate from the training set using MI [106] to obtain the learned probabilistic shape model $P_{\mathbf{s}}(\mathbf{m})$.

2. Estimate the conditional intensity model $P(\mathbf{g} \mid \mathbf{m})$ by identifying the bimodal LCDG.

3. Use the intensity model that is estimated using LCDG and the learned probabilistic shape model to perform an initial segmentation (initial region map) of the prostate.

4. Use the initial region map to estimate the potential for the Potts model using Equation (5) and to identify the MGRF model $P(\mathbf{m})$ of region maps.

5. Improve the region map using voxel-wise stochastic relaxation (Iterative conditional mode-ICM [107]) through successive iterations to maximize the log likelihood function of Equation (2) until the log likelihood remains almost the same for two successive iterations.

6. Update the Shape Prior: by adding the current segmented 3D prostate data to the prior calculated shape model. 


\section{Nonrigid Registration}

Due to patient breathing and local movement, accurate registration is a main issue in DCE-MRI sequences. A tremendous number of nonrigid image registration techniques have been developed, e.g., [60,63]. However, more robust, efficient, and sophisticated registration techniques are required. In order to avoid problems associated with intensity variations over the temporal dynamic contrast agent data set, the proposed approach exploits geometric features, rather than image intensities. These geometric features are estimated from the electric field vectors that are calculated by solving the Laplace second-order PDE between the segmented prostate borders. Estimating these field vectors allow for co-allocation of point-to-point correspondences between the segmented prostate objects. Mathematically, the second-order Laplace PDE defines a scalar field $\gamma$, defined as:

$$
\nabla^{2} \gamma=\frac{\partial^{2} \gamma}{\partial x^{2}}+\frac{\partial^{2} \gamma}{\partial y^{2}}=0
$$

The solution $\gamma(x, y)$ of Equation (6) results in intermediate equipotential surfaces and streamlines (field lines) that are everywhere orthogonal to all equipotential surfaces and establish natural point-to-point correspondences between the boundaries (see e.g., the line connecting the points $\mathbf{B}_{a i}$ and $\mathbf{B}_{b j}$ in Figure. 24). In medical imaging applications, the Laplace-based approaches have been previously used e.g., for colon surface flattening and centerline extraction [108] and thickness measurements [109-111]. To the best of our knowledge, the Laplace-potentialbased method is the first of its kind to be applied to the motion correction in DCE-MRI images for further improved analysis of the DCE-MRI data. The proposed Laplace-based nonrigid registration approach is based on deforming each pixel of the segmented prostate objects over a set of nested, equi-spaced contours 
(i.e., iso-contours), which is generated for both the target and reference prostate objects as shown in Figure 23. To find the iso-contours, a distance map is generated inside the binary object area by finding the minimum Euclidean distance for every inner point to the object boundary. The external points are excluded from consideration. Then, the Laplace equation is applied to the respective reference and target iso-contours to co-locate their corresponding points. The basic steps of the Laplace-potential-based nonrigid registration are summarized in Algorithm IV.B.2.

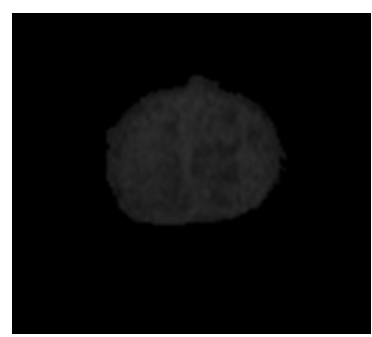

(a)

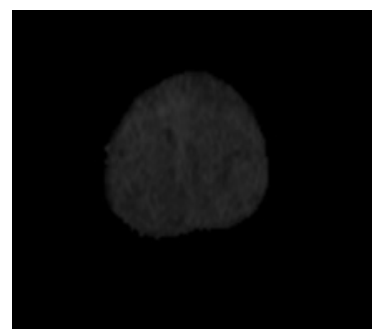

(d)

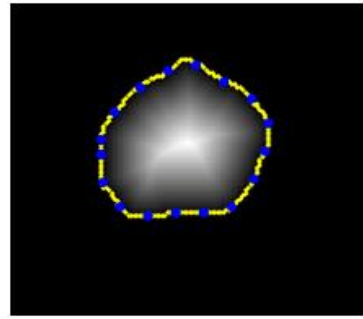

(b)

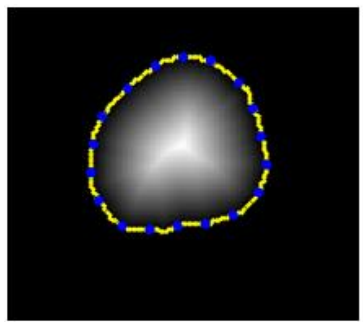

(e)

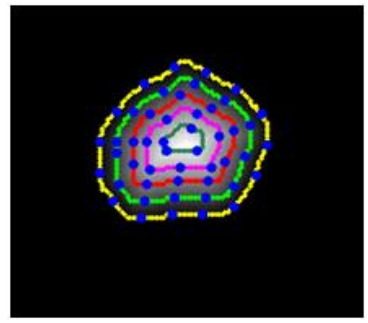

(c)

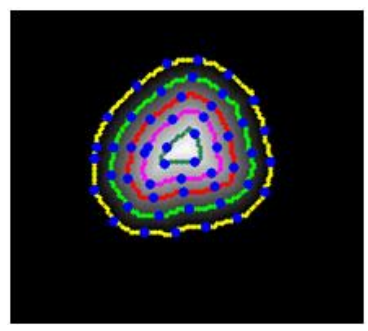

(f)

FIGURE 23: Illustration of the iso-contours generation: the reference (a) and target (d) images, the reference (b) and target (e) distance maps, and reference (c) and target (f) iso-contours. 
Algorithm 3 Laplace-Based Iso-Contours Nonrigid Registration

Input: segmented prostate objects.

Output: point-to-point correspondences.

1. Generate the distance maps inside the segmented prostate as shown in Figure $23(b, e)$.

2. Generate nested, iso-contours for both target and reference distance maps as shown in Figure. 23 (c, f).

3. Initial condition: Set the potential $\gamma$ maximum at the target iso-contour and minimum (equal zero) at the reference iso-contour.

4. Solve Eq. (6) between respective iso-contours using the above initial condition.

5. Compute components of the gradient vectors $E_{x}=\frac{\partial \gamma}{\partial x}$ and $E_{y}=\frac{\partial \gamma}{\partial y}$.

6. Find the point-to-point correspondences between the iso-contours being matched by forming the streamlines.

7. Repeat steps 3 to 6 for the next set of corresponding iso-contours. 


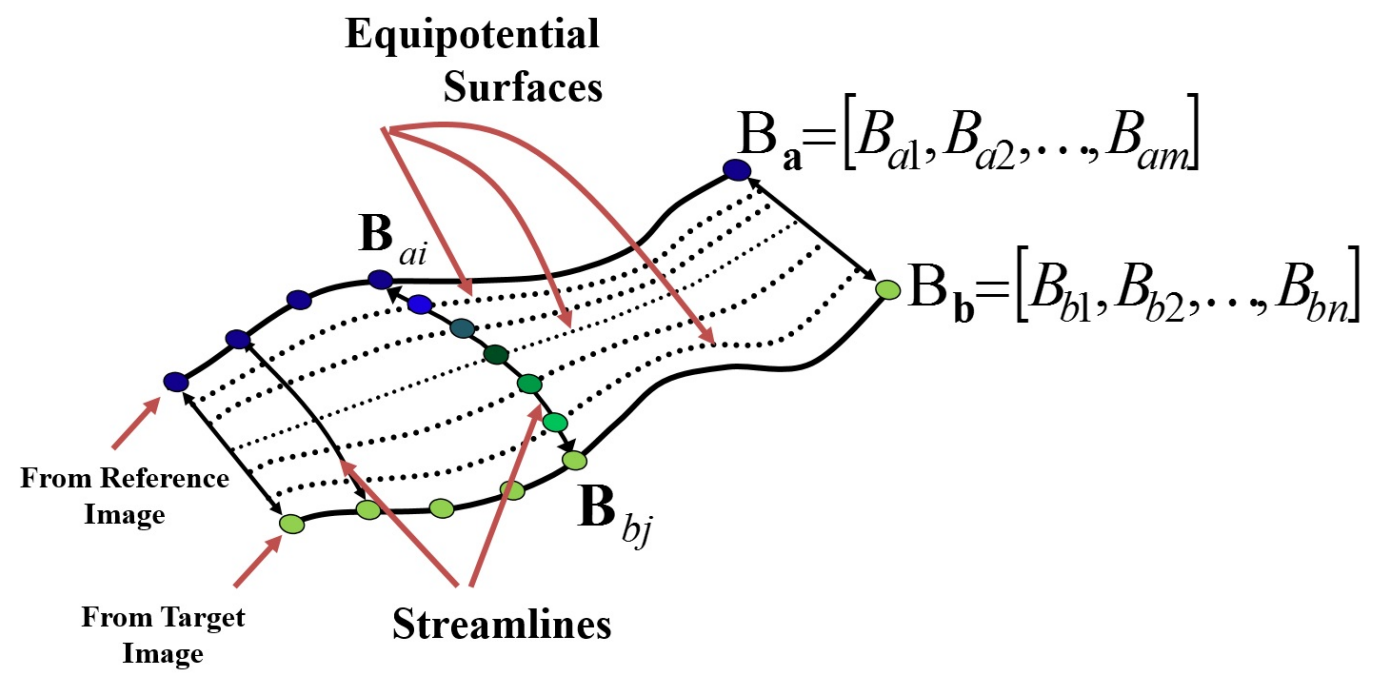

FIGURE 24: 2-D schematic illustration of co-allocation of the point-to-point correspondences between two boundaries by a potential field.

3. Classification, Performance Analysis, and Visualization of Perfusion Indexes

After the nonrigid alignment, the wash-in and wash-out curves are constructed by calculating the average intensities of prostate regions for each time sequence. These curves show the response of the prostate tissues as the contrast agent perfuses for each image section (see Figure 32).

a. Color Map Generation and Tumor Boundary Determination To characterize the physiological data, color-coded maps that illustrate the propagation of the contrast agent in the prostate tissues are constructed. To construct the initial color maps, we have to estimate the changes in image signals $\delta_{x, y, z}$ due to the contrast agent. These changes are estimated from the constructed perfusion curves as the difference between the signals of image sequences at $t_{p}$ and $t_{0}$ (see Figure. 25).

To preserve continuity (remove inconsistencies), the initial estimated $\delta_{x, y, z}$ values are considered as samples from a Generalized Gauss-Markov Random Field 


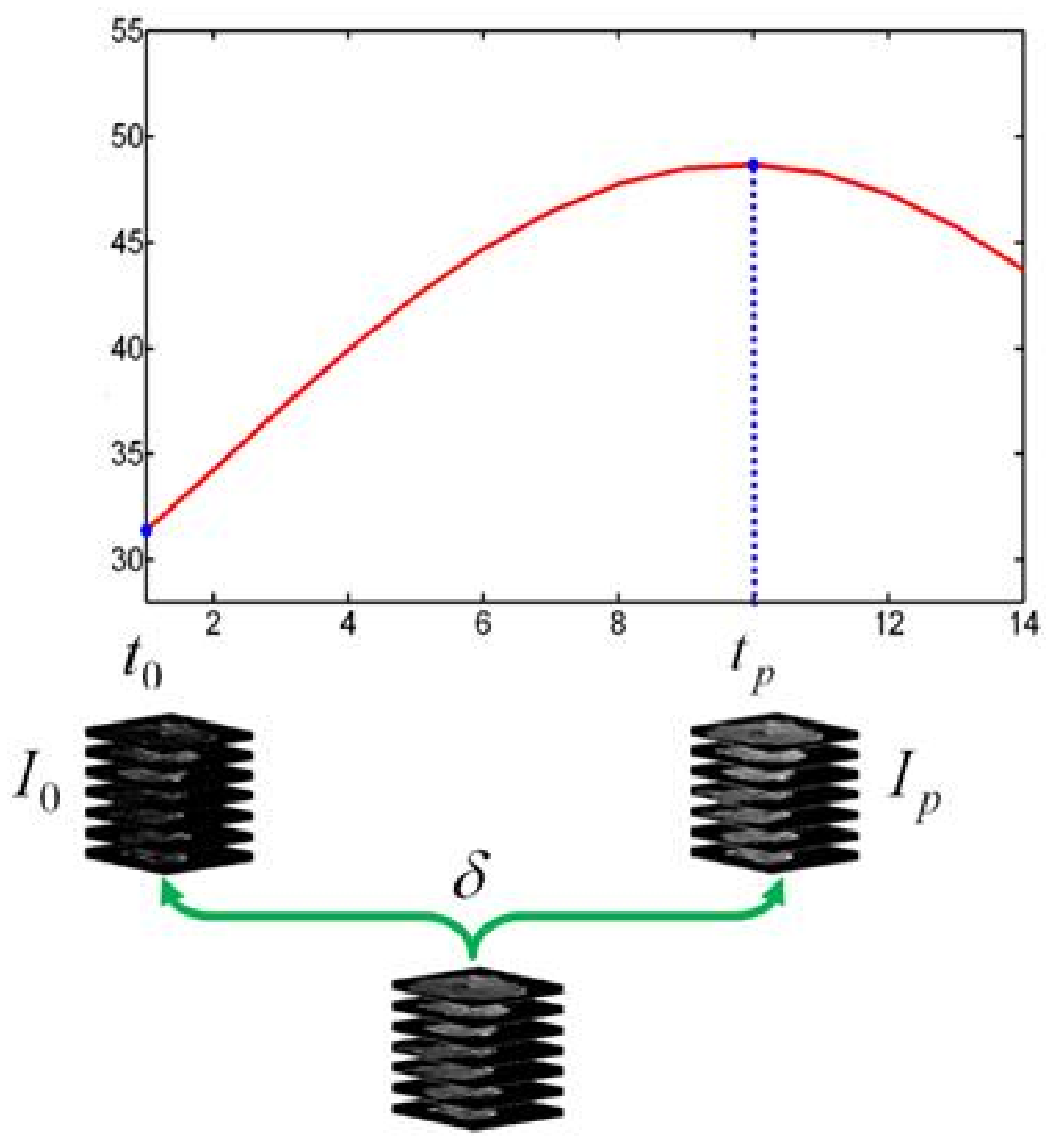

FIGURE 25: Estimating $\delta$ from the perfusion curve as the difference between the peak and initial signal of image sequences.

(GGMRF) image model of measurements with the 26-voxel neighborhood (Figure 18). Continuity of the constructed 3-D volume (Figure 26) is amplified by using their Maximum a Posteriori (MAP) estimates as shown in [112]:

$\widehat{\delta}_{x, y, z}=\arg \min _{\widetilde{\delta}_{x, y, z}}\left\{\left|\delta_{x, y, z}-\widetilde{\delta}_{x, y, z}\right|^{\alpha}+\rho^{\alpha} \lambda^{\beta} \sum_{\left(x^{\prime}, y^{\prime}, z^{\prime}\right) \in \nu_{(x, y, z)}} \eta_{(x, y, z),\left(x^{\prime}, y^{\prime}, z^{\prime}\right)}\left|\widetilde{\delta}_{x, y, z}-\delta_{x^{\prime}, y^{\prime}, z^{\prime}}\right|\right\}$

where $\delta_{x, y, z}$ and $\widetilde{\delta}_{x, y, z}$ denote the original values and their expected estimates, $\nu_{(x, y, z)}$ is the 26-neighborhood voxel set (Figure 18), $\eta_{.,}$. is the GGMRF potential, and $\rho$ and 


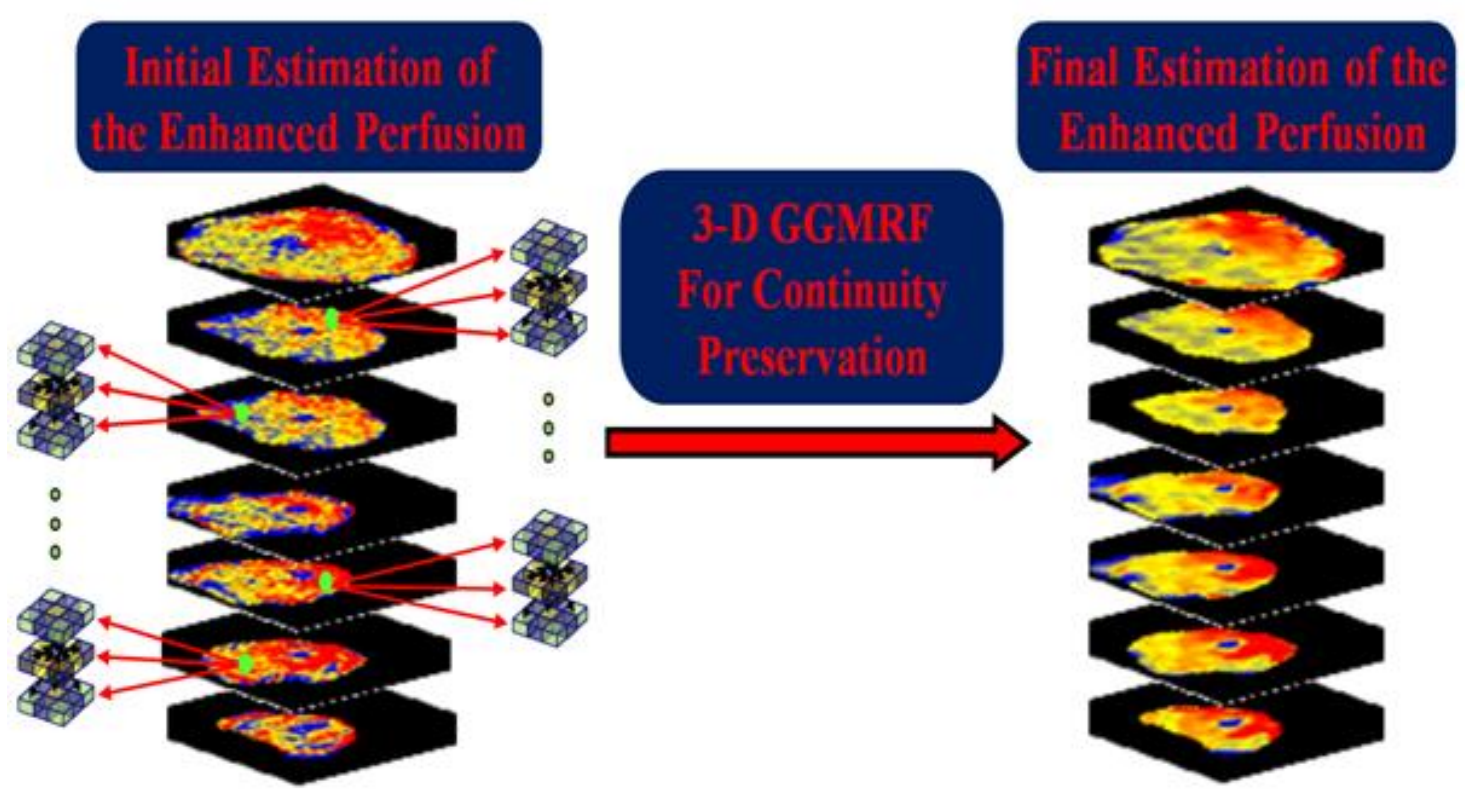

FIGURE 26: Enhanced perfusion estimation and continuity analysis using the 3D GGMRF image model.

$\lambda$ are scaling factors. The parameter $\beta \in[1.01,2.0]$ controls the level of smoothing (e.g., smooth, $\beta=2$, vs. relatively abrupt edges, $\beta=1.01$ ). The parameter $\alpha \in\{1,2\}$ determines the Gaussian, $\alpha=2$, or Laplace, $\alpha=1$, prior distribution of the estimator. Then, the color maps are generated based on the final estimated $\widehat{\delta}$ (see Figure 33).

Finally, to allocate the boundary of the detected tumors, which is important to determine the cancer stage in case of malignancy, we used a level set-based deformable model controlled by a stochastic speed function [113]. The latter accounts for the perfusion information and spatial interactions between the prostate voxels.

\section{PERFORMANCE EVALUATION AND VALIDATION}

To evaluate the performance of the proposed framework, we used two types 
of error metrics, the voxel-based and distance-based errors. To calculate the voxelbased error, we measure the True Positive (TP), True Negative (TN), False Positive (FP), and False Negative (FN) segmentation. Let $|\mathbf{C}|,|\mathbf{G}|$, and $|\mathbf{g}|$ denote volumes (by the number of voxels) of the segmented volume, C, its ground truth, G, and the MR data, $\mathbf{g}$, respectively. Then $\mathrm{TP}=|\mathbf{C} \cap \mathbf{G}|$ is the overlapping between $\mathbf{C}$ and $\mathbf{G} ; \mathrm{TN}=|\mathbf{g}-\mathbf{C} \cup \mathbf{G}| ; \mathrm{FP}=|\mathbf{C}-\mathbf{C} \cap \mathbf{G}|$, and $\mathrm{FN}=|\mathbf{G}-\mathbf{C} \cap \mathbf{G}|$.

The Dice similarity coefficient (DSC) measures set agreement between two sets $\mathbf{C}, \mathbf{G}$ and is defined as the union size of the two sets divided by the average size of the two sets:

$$
D S C(C, G)=\frac{2|\mathbf{C} \cap \mathbf{G}|}{\mathbf{C} \cap \mathbf{G}+\mathbf{C} \cup \mathbf{G}}
$$

We calculate the Positive Predictive Value (PPV), Sensitivity (Sens), and Dice Coefficient (DSC) - as defined as [114]:

$$
\begin{aligned}
P P V & =\frac{T P}{T P+F P} \\
\text { Sens } & =\frac{T P}{T P+F N} \\
D S C & =\frac{2 * T P}{2 * T P+F P+F N}
\end{aligned}
$$




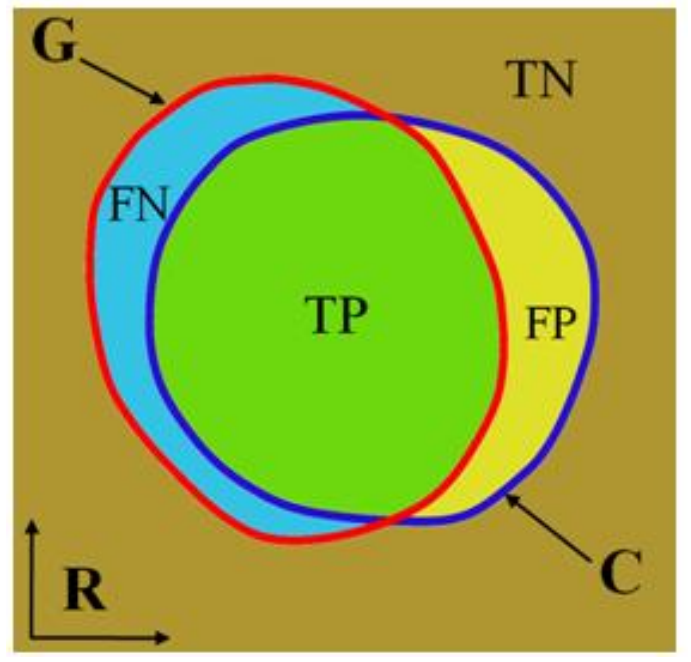

FIGURE 27: 2-D schematic illustration of measuring segmentation errors between the ground truth $\mathrm{G}$ and automatic segmentation $\mathrm{C}$ obtained by the our segmentation approach.

\section{PATIENT DATA AND DCE-MRI ACQUISITION PROTOCOL}

The performance of the proposed segmentation approach is evaluated by applying it on 3D DCE-MRI prostate images. We observed that good selection of a DCE-MRI imaging protocol is as important as the image analysis. The protocol described below has been found to be optimal with the current MRI hardware (Signa Horizon LX Echo speed; General Electric Medical Systems, Milwaukee, WI, USA).

In our protocol, gradient-echo T1 imaging was employed by a Signa Horizon GE 1.5 Tesla MR scanner using an additional pelvic coil. Images were taken at $7 \mathrm{~mm}$ thickness with an interslice gap of $0.5 \mathrm{~mm}$. The repetition time (TR) was $50 \mathrm{~ms}$, the $T_{E}$ was minimum with flip angle at $60^{\circ}$ degrees, the band width was 31.25 kilohertz $(\mathrm{kHz})$, the field of view $(\mathrm{FOV})$ was $28 \mathrm{~cm}$, and the number of slices was 7. The DCE-MRI process started with a series of MRI scans which were used 
to establish a baseline in image intensity. These scans were performed without the administration of contrast enhancing agents so that the tissue's nonenhanced image intensity could be established. In the next stage, 10 cubic centimeter $(c c)$ of gadoteric acid (Dotarem 0.5 millimole/milliliter $(\mathrm{mmol} / \mathrm{mL})$; Guerbet, France) was administered intravenously at a rate of $3 \mathrm{~mL} / \mathrm{s}$. At this point, a series of MRI scans was performed every 3 seconds for 6 minutes, and every series contained 7 slices. Note that all the subjects were diagnosed using a biopsy (ground truth).

\section{Prostate Segmentation Results}

The proposed segmentation approach has been tested on DCE-MRI sequences for 30 independent subjects. Figure 28 demonstrates some segmentation results of the prostate region at selected image sections for different subjects and their associated False Positive (FP) and False Negative (FN) errors. For comparison, our segmentation results are compared to the radiologists tracing based on the Positive Predictive Value (PPV), Sensitivity (Sens), and Dice Similarity Coefficient (DSC) [114] statistics for the proposed approach are summarized in Table 4. To highlight the advantage of integrating the shape prior with the intensity and spatial interaction information in the joint MGRF probabilistic model, we segment the prostate region based only on the intensity and spatial interaction information. The segmentation results are shown in Figure 29 (d). It is clear that counting on intensity and spatial interaction information only will not lead to accurate segmentation due to the gray-levels inhomogeneities. 

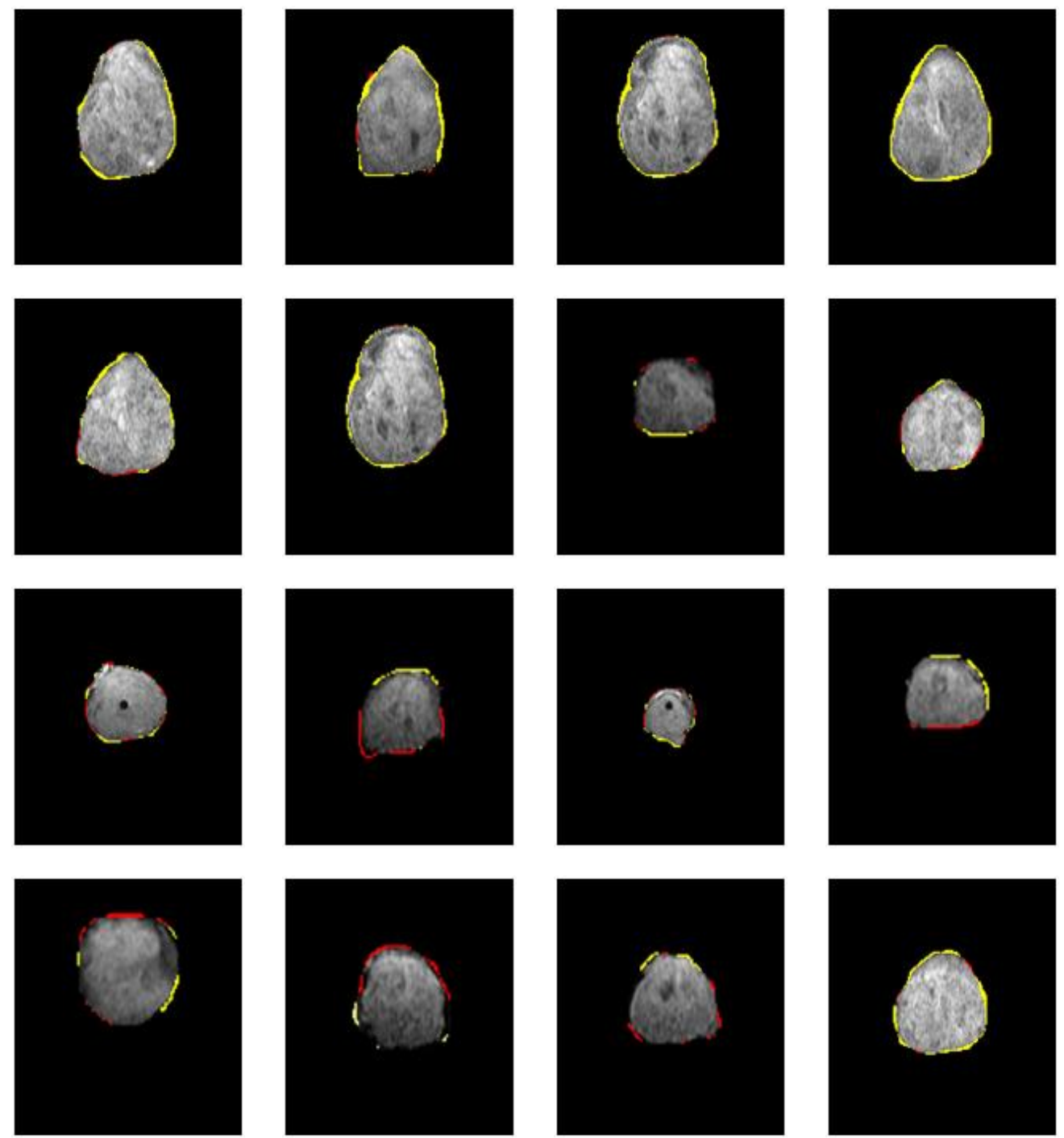

FIGURE 28: Segmentation results: the segmentation results with error referenced to the ground truth $G$ is outlined in yellow (False Negative (FN): pixels segmented as the prostate in $G$ but not segmented as the prostate with our approach) and red (False Positive (FP): pixels segmented as the prostate with our approach but not segmented as the prostate in the $G$. 
TABLE 4: Error statistics of the proposed segmentation approach. Note that SD, stands for standard deviation.

\begin{tabular}{|c|c|c|c|}
\cline { 2 - 4 } \multicolumn{1}{c|}{} & PPV & SEN & DSC \\
\hline Mean \pm SD & $0.98 \pm 0.004$ & $0.846 \pm 0.004$ & $0.923 \pm 0.004$ \\
\hline
\end{tabular}

TABLE 5: Comparative segmentation accuracies of the proposed prostate segmentation against the shape-based (SB) approach [2] in comparison to the experts ground truth (SD standard deviation).

\begin{tabular}{|l|c|c|}
\cline { 2 - 3 } \multicolumn{1}{c|}{} & \multicolumn{2}{c|}{ Segmentation Technique } \\
\cline { 2 - 3 } \multicolumn{1}{c|}{} & Proposed Approach & Shape-based(SB) approach [2] \\
\hline Mean \pm SD & $0.53 \pm 0.33$ & $5.91 \pm 4.44$ \\
\hline Two-tailed P-value & \multicolumn{2}{|c|}{0.0001} \\
\hline
\end{tabular}

To show the advantage of the proposed segmentation approach, all time series images have been segmented using the shape-based approach proposed in Tsai et al. [2]. The comparative results for a few of them are shown in Figure 30. Table 5 compares the segmentation results over all test data sets with the known ground truth (manual tracing by an imaging expert) and the differences are shown to be statistically significant by the unpaired t-test (the two-tailed value $\mathrm{P}$ is less than 0.0001). 

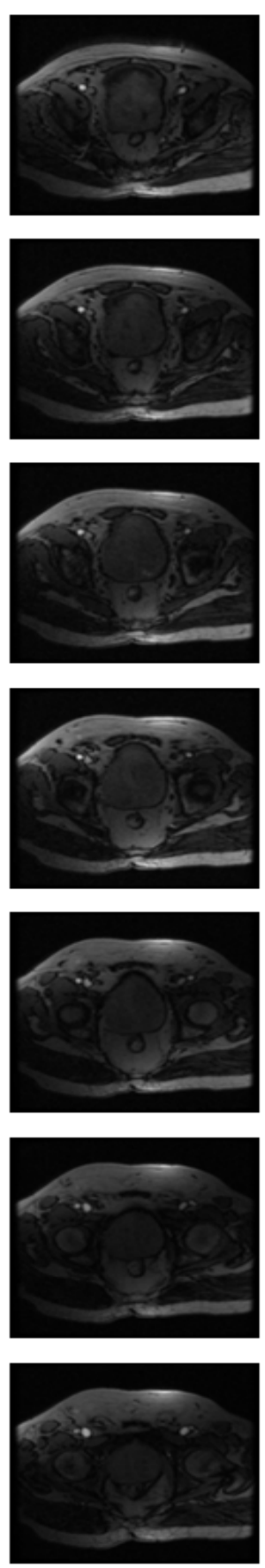

(a)
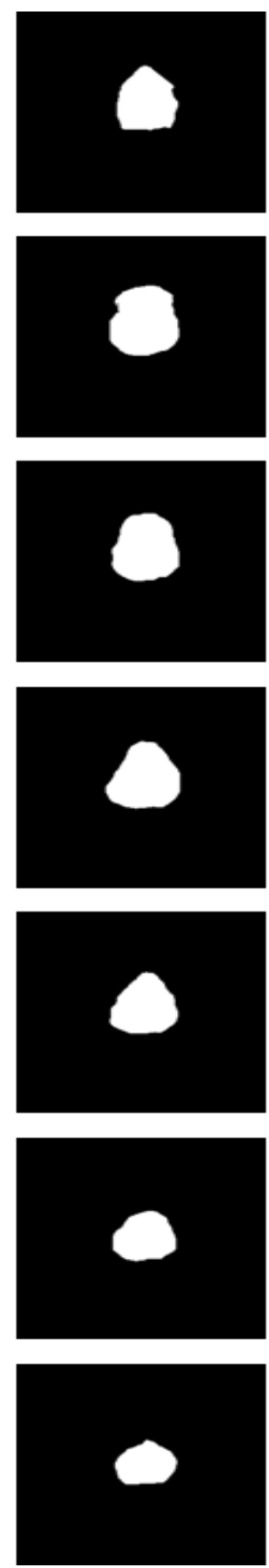

(b)
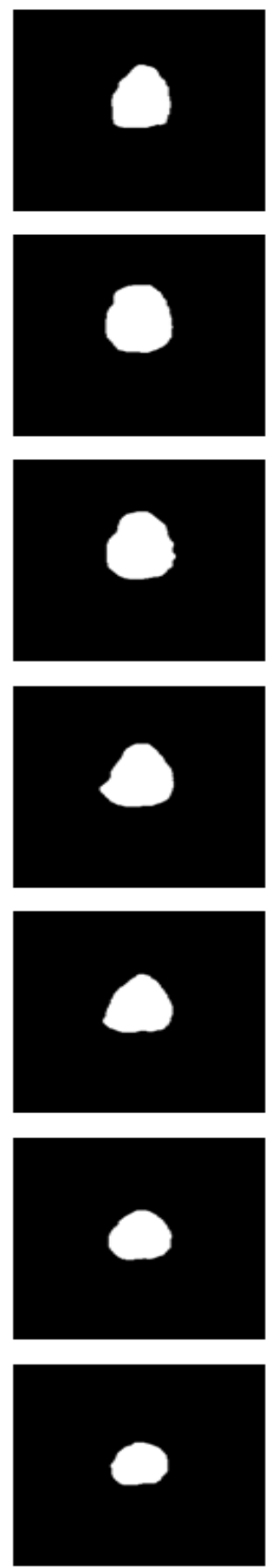

(c)
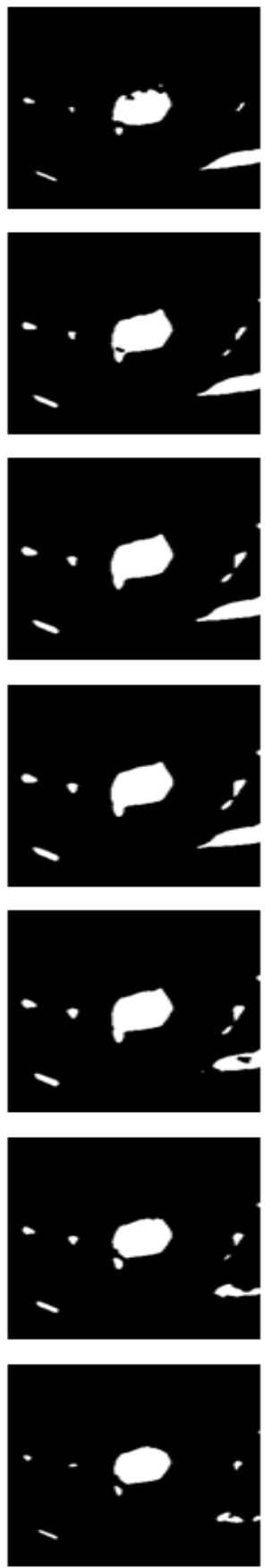

(d)

FIGURE 29: Segmentation results of the first subject after 30 iteration: (a) typical prostate DCE-MRI images. (b) the ground truth (c) segmentation results by intensity, shape prior, and spatial interaction (d) segmentation results by intensity, and spatial interaction only. 

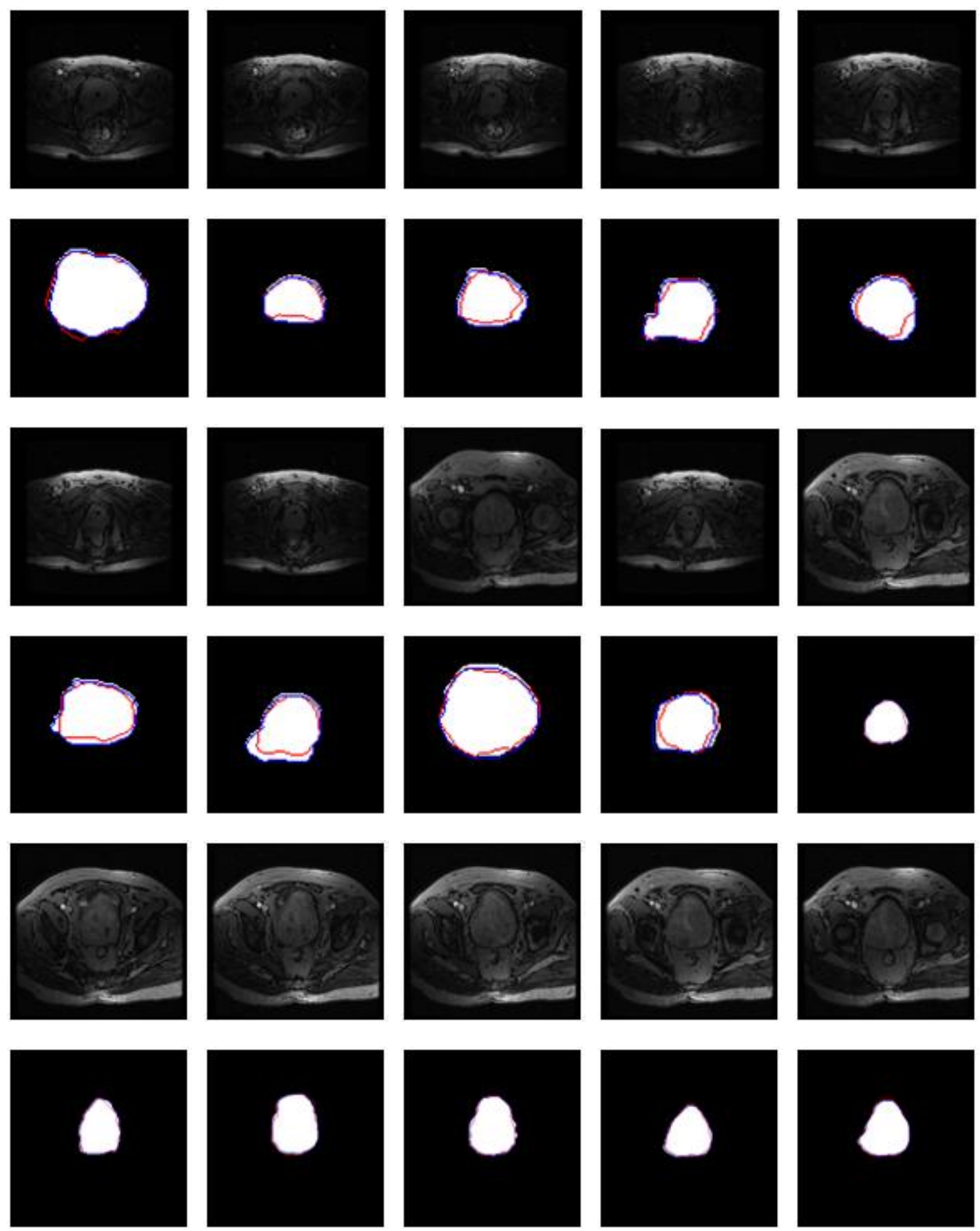

FIGURE 30: Accuracy of our segmentation in comparison with [2]. Our segmentation is outlined in blue and [2] in red with reference to the ground truth $G$ in white. 
2. Diagnostic Results

The ultimate goal of the proposed framework is to successfully distinguish malignant from benign detected tumors by constructing the perfusion curves from the DCE-MRI sequences (see Figure 31).

(a)

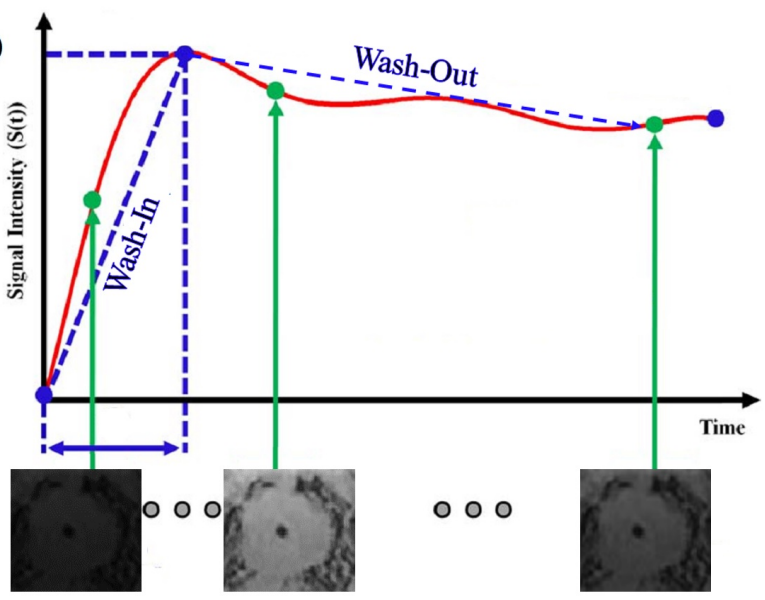

(b)

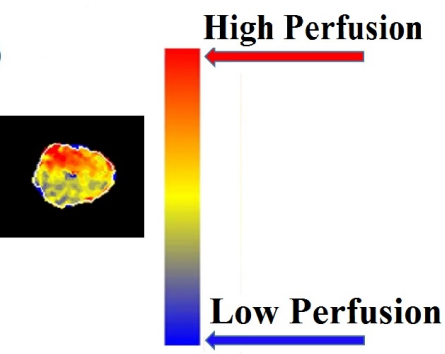

FIGURE 31: Estimation of the perfusion parameters for the classification of prostate tumor (a) Dynamic sequence showing prostate-averaged voxel intensity versus time, along with images at three different time (b) Prostate colored by washin and wash-out.

The curves on Figure 32 show the response of the prostate tissues as the contrast agent perfuses. The malignant subjects show an abrupt increase to the higher perfusion values and the benign subjects show a delay in reaching their peak perfusion (see Figure 32).

From these curves, we conclude that the peak signal value and the washin slope are the two major features that can be extracted for the classification of prostate cancer. To distinguish between benign and malignant cases, a $k_{n}$-Nearest Neighbor classifier is used to learn the statistical characteristics of both benign and malignant cases from the time-intensity curves of the training sets (see Figure 32). The diagnostic accuracy of the $k_{n}$ classifier was $98 \%$ using Leave One-Subject-Out 


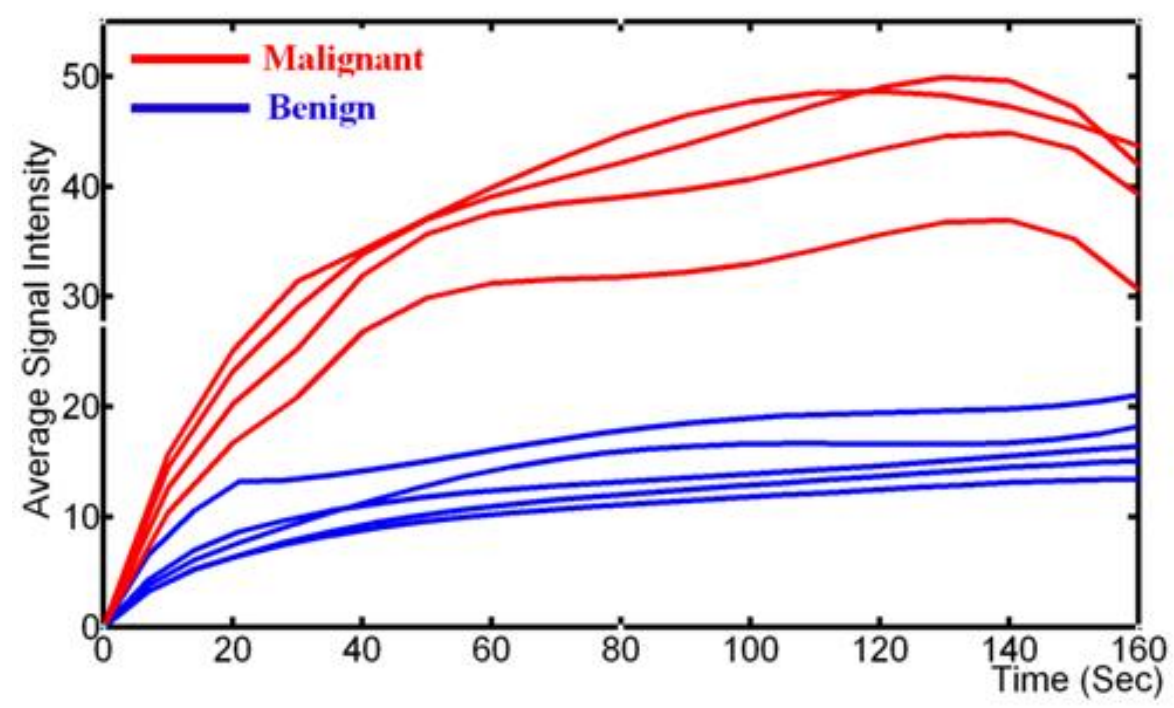

FIGURE 32: Selected normalized signal intensity, averaged over the entire prostate, with respect to the timing of contrast agent delivery for malignant (red) and benign (blue) subject.

(LOSO) approach.

Following the classification, a visual assessment is made. Figure 33 presents the color-coded maps over all image sections before and after applying the 3-D GGMRF smoothing for three subjects involved in our study. Figures 34 and 36 show two examples of the tumor contours, determination for benign and malignant subjects. 


\begin{tabular}{|c|c|c|c|c|c|c|c|}
\hline \multicolumn{2}{|c|}{ Subject\# 1 } & \multicolumn{2}{c|}{ Subject\# 2 } & \multicolumn{2}{c|}{ Subject\# 3 } & \multicolumn{2}{c|}{ Subject\# 4 } \\
\hline Before & After & Before & After & Before & After & Before & After \\
Max \\
(n)
\end{tabular}

FIGURE 33: Color-coded maps for four of the test subjects before and after the 3-D GGMRF smoothing with $\rho=1, \lambda=5, \beta=1.01, \alpha=2$, and $\eta_{s, r}=\sqrt{2}$ and their respective color-coded maps. The red and blue ends of the color scale relate to the maximum and minimum changes, respectively. 

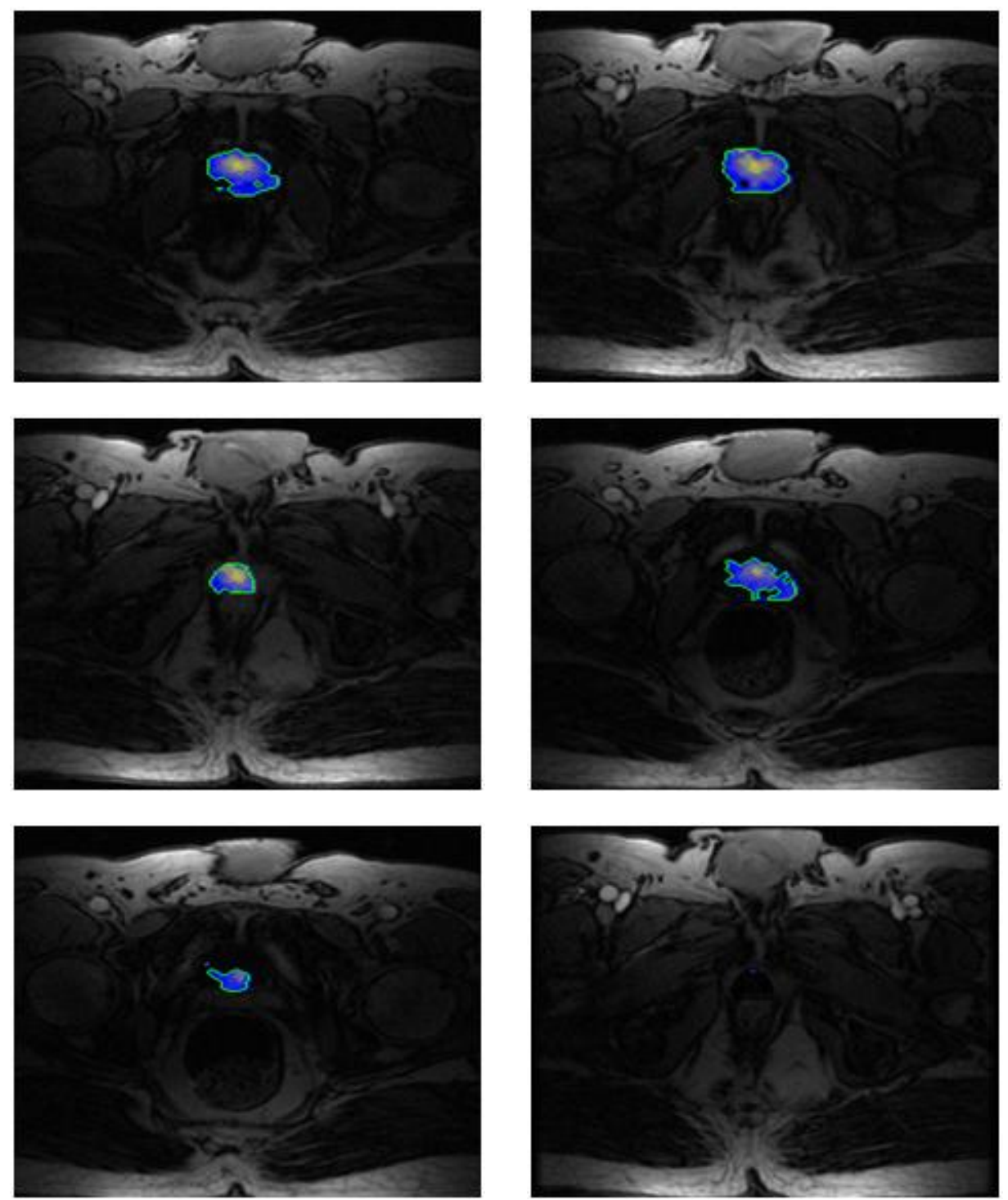

FIGURE 34: Color-coded maps of local tumor progression overlaid on anatomic DCE-MRI data for one benign subject. The determined tumor contours are shown in blue. 

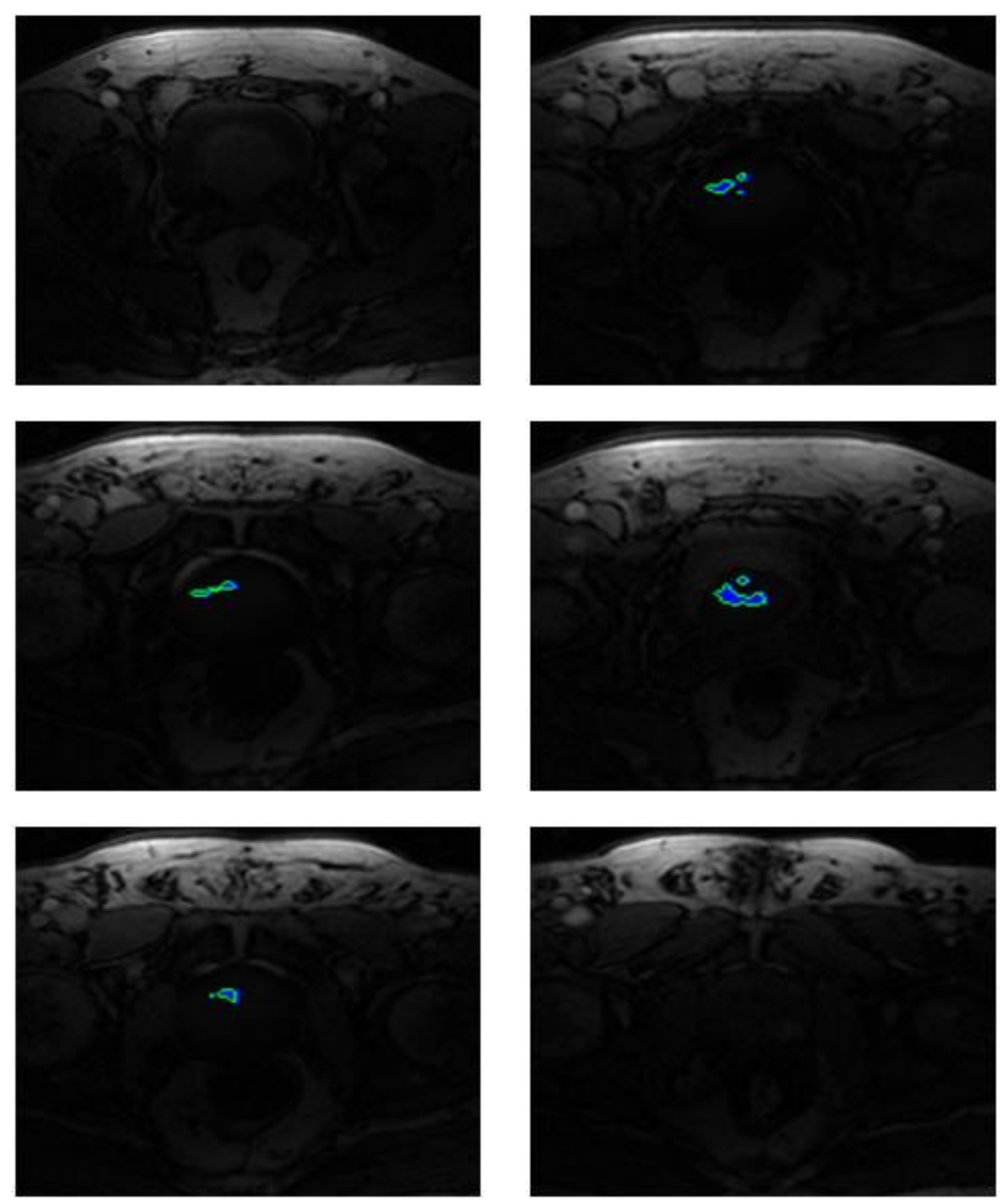

FIGURE 35: Color-coded maps of local tumor progression overlaid on anatomic DCE-MRI data for one benign subject. The determined tumor contours are shown in blue. 

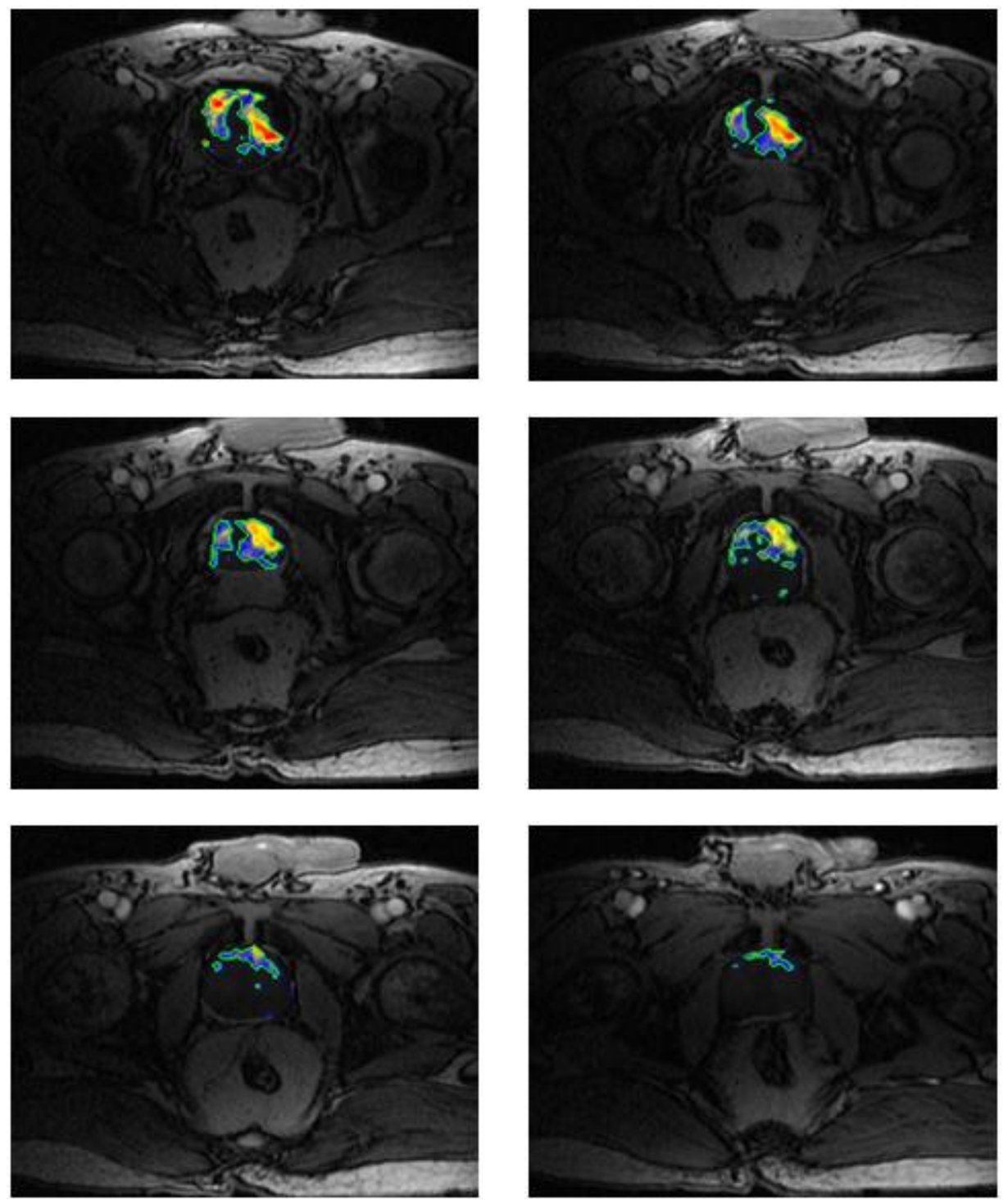

FIGURE 36: Dynamic contrast-enhanced MRI images of the pelvis with local tumor progression of malignant subject. The determined tumor contours are shown in blue. 

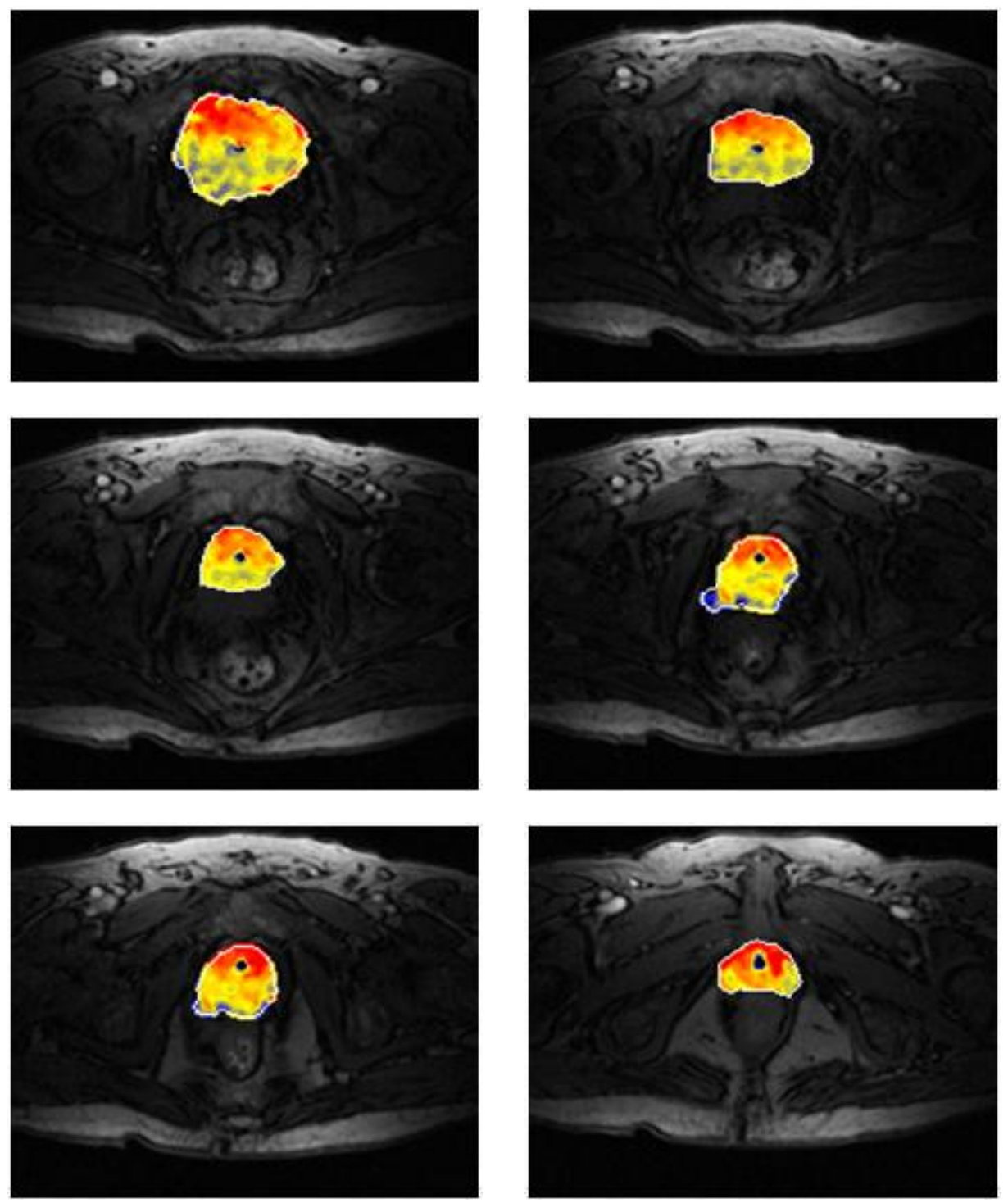

FIGURE 37: Dynamic contrast-enhanced MRI images of the pelvis with local tumor progression of another malignant subject. The determined tumor contours are shown in blue. 


\section{E. SUMMARY}

In this chapter, we presented a noninvasive MRI-based CAD for detecting prostate cancer. The proposed framework demonstrated the documented ability to reliably distinguish malignant from benign, in a biopsy-proven preliminary cohort of 30 participants. The proposed framework includes prostate segmentation, nonrigid registration, and $k_{n}$-Nearest Neighbor based classification. For prostate segmentation, we introduced a new 3D approach that is based on a MAP estimate of a new log-likelihood function that accounts for the shape priori, the spatial interaction, and the current appearance of the prostate tissues and its background. Following segmentation, we introduced a nonrigid registration approach that deforms the prostate object on iso-contours instead of a square lattice, which provides more degrees of freedom to obtain accurate deformation. The perfusion curves of the segmented prostate region are calculated and the features extracted from these curves undergo $k_{n}$-Nearest Neighbor based classification. Applications of the proposed approach yield promising results that would, in the near future, replace the use of current technologies to determine the type of prostate cancer. The work presented in this chapter has been published in the IEEE International Symposium on Biomedical Imaging (ISBI), [52], Computational Intelligence in Biomedical Imaging [30], and the Medical Image Computing and Computer-Assisted Intervention (MICCAI) conference [51] 


\section{CHAPTER V}

\section{A NOVEL IMAGE-BASED APPROACH FOR EARLY DETECTION OF PROSTATE CANCER USING DIFFUSION-WEIGHTED MRI}

This chapter introduces a fully automatic non-invasive approach for the early diagnosis of prostate cancer using Diffusion-Weighted MRI (DWI). The proposed diagnostic approach consists of the following four steps to detect locations that are suspicious for prostate cancer: 1) In the first step, we isolate the prostate from the surrounding anatomical structures based on a Maximum A Posteriori (MAP) estimate of a new log-likelihood function that ac- counts for the shape priori, the spatial interaction, and the current appearance of prostate tissues and its background (surrounding anatomical structures); 2) In order to take into account any local deformation between the segmented prostates at different $b$-values that could occur during the scanning process due to local motion, a non-rigid registration algorithm is employed; 3) A $k_{n}$-Nearest Neighbor classifier is used to classify the prostate into benign or malignant based on three appearance features extracted from registered images; and 4) The tumor boundaries are determined using a level set deformable model controlled by the diffusion information and the spatial interactions between the prostate voxels. Experimental results on in vivo data confirm the accuracy and robustness of our method. 


\section{A. INTRODUCTION}

Earlier studies [40] have investigated the abilities of DWI for prostate cancer diagnosis using an endorectal coil. However, the reported results demonstrated low diagnostic sensitivity [115]. To increase the sensitivity of diagnosis, Shimofusa et al. [34] suggested the addition of strong magnetic field gradient pulses (b-values) to the pulse sequence instead of using an endorectal coil. In their study [34], they detected the tumor in the central zone of the prostate in five of eight total patients using DWI with strong magnetic field gradient pulses. Alternatively, the compared diagnostic results with $T_{2}$-weighted imaging, detected the tumor only in one of the eight patients. Since then, DWI were used for the detection of cancerous tissue in later studies $[36,38,39,41,116]$. For example, Tan et al. [116] compared the performance of $T_{2}$-weighted MRI, DCE-MRI, and DWI for the detection of cancer within the prostate gland. In their study, they reported that DWI alone showed better specificity than DCE-MRI alone. It is also showed better overall specificity than combined DWI and $T_{2}$-weighted imaging. To the best of our knowledge, there is a very limited number $[38,41]$ of image-based approaches for automated computer-aided diagnosis of prostate cancer using DWI. Therefore, there is a need for developing new methods for automated computer-aided diagnosis of prostate cancer using DWI.

\section{B. MATERIALS AND METHODS}

In this section we introduce automated and non-invasive framework for early diagnosis of prostate cancer using DWI. Figure 38 demonstrates the steps of the proposed CAD system. Below, we will illustrate each of these steps. 


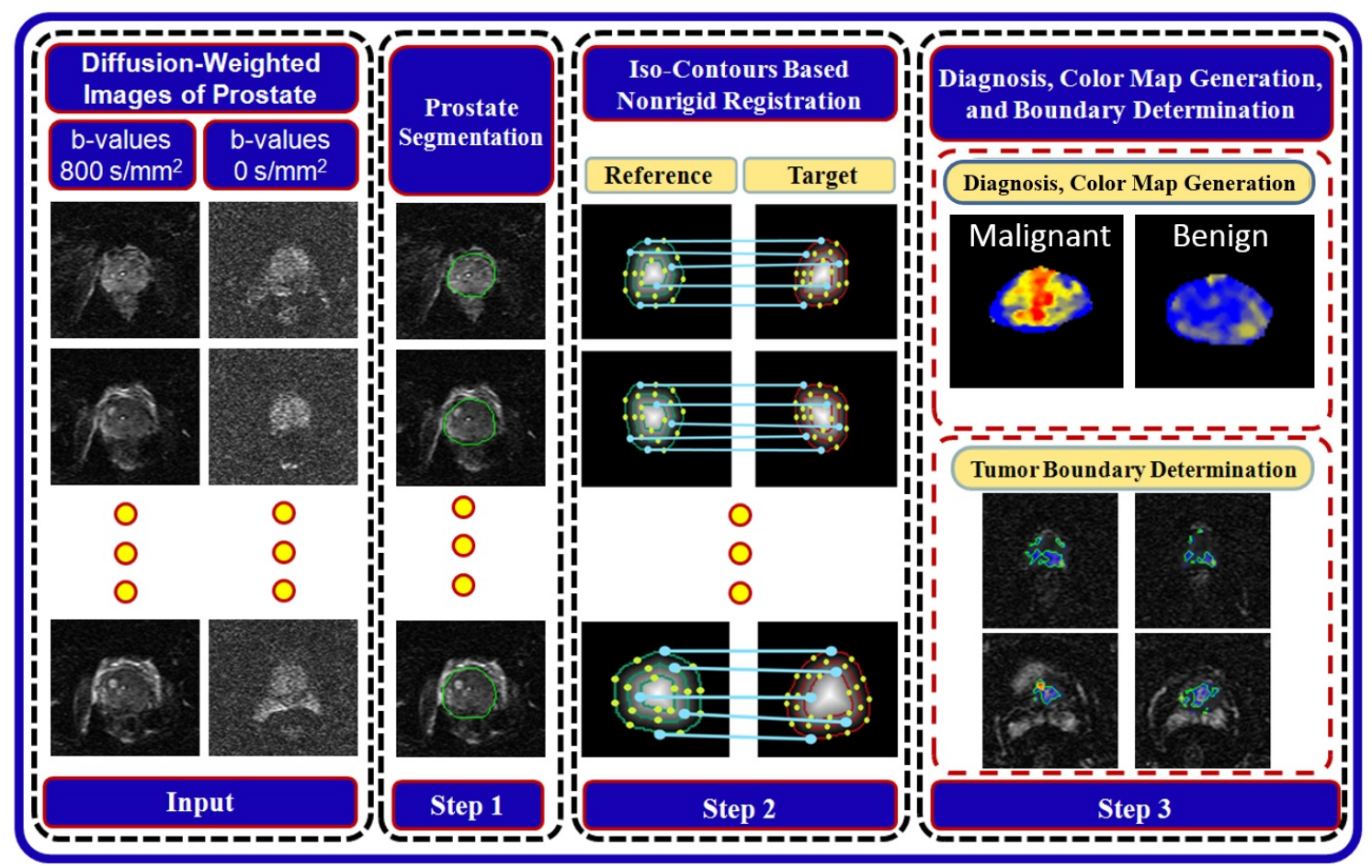

FIGURE 38: Flowchart of the proposed CAD system for automatic detection of cancer from 3D DWI.

1. Delineation of the Prostate Borders

The segmentation of the prostate from DWI is a challenge due to the anatomical complexity of the prostate and the undistinguishable gray-level distribution of the prostate and surrounding organs. To account for these challenges, a MAPbased approach based on a learned shape model and an identifiable joint MarkovGibbs random field (MGRF) model is proposed. The proposed MGRF image model relates the joint probability of an image and its object-background region segmentation map, to geometric structure and to the energy of repeated patterns within the image [117]. The basic theory behind such models is that they assume that the signals associated with each voxel depend on the signals of the neighboring voxel, and thus explicitly take into account their spatial interaction, and other features, 
such as shape.

The input 3D DWI data $g$ and its region map $m$ are described by the joint MGRF model as outlined in Section IV.B.1, Equation 1. The Bayesian MAP estimate of the map, given $\mathbf{g}, \mathbf{m}^{*}=\arg \max _{\mathbf{m}} L(\mathbf{g}, \mathbf{m})$ maximize the log-likelihood function:

$$
L(\mathbf{g}, \mathbf{m})=\log (P(\mathbf{g} \mid \mathbf{m}))+\log \left(P_{\mathrm{h}}(\mathbf{m})\right)
$$

where $P(\mathbf{g} \mid \mathbf{m})$ is a conditional distribution of the images given the map $m$ and $P(\mathbf{m})=P_{\mathrm{sp}}(\mathbf{m}) P_{\mathrm{v}}(\mathbf{m})$ is an unconditional probability distribution of maps. Here, $P_{\mathrm{sp}}(\mathbf{m})$ denotes the prostate shape prior, and $P_{\mathrm{v}}(\mathbf{m})$ is a Gibbs probability distribution with potentials $V$, which specifies a MGRF model of spatially homogeneous maps $m$.

The specific visual appearance of the prostate in each data set to be segmented is taken into account by modeling the marginal gray level distribution with the LCDG model [91-93] as described in Section IV.B.1.a. To overcome noise effect and to ensure the homogeneity of the segmentation, the spatial voxel interactions between the region labels of a given map $m$ are also taken into account using the pairwise MGRF spatial model as described in Section IV.B.1.b and the nearest voxel 26-neighbors shown in Figure 39.

In addition to voxel-wise image intensities and their pairwise spatial interaction, additional constraints based on the expected shape of the prostate are introduced by co-aligning each given DWI data to a training database and using probabilistic 3D prostate shape model $P_{\mathrm{sp}}(\mathbf{m})$. To perform initial prostate segmentation, a given 3D DWI is aligned to one of the training data. The shape model provides the voxel-wise object and background probabilities being used together with 


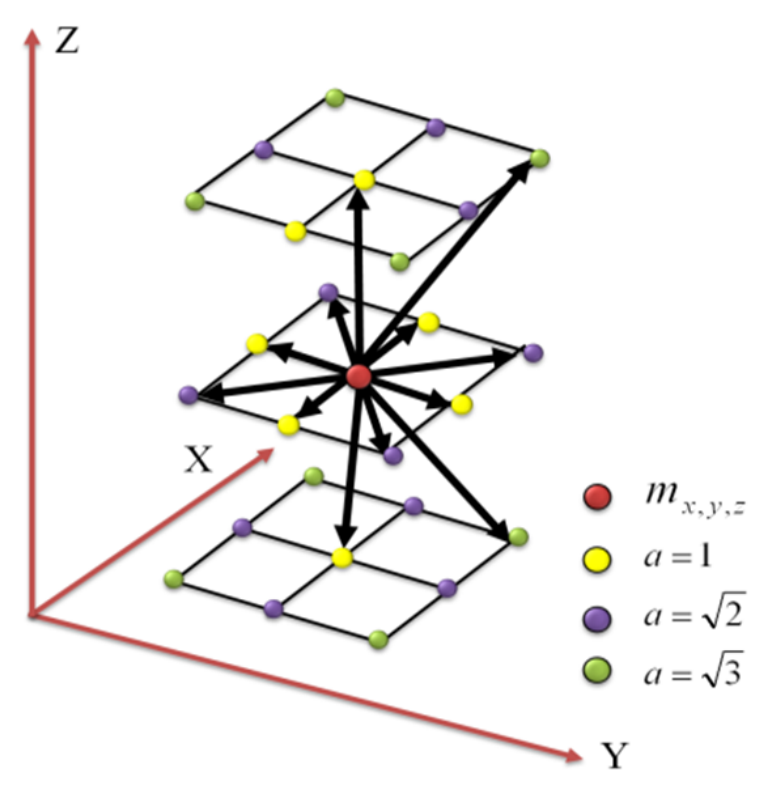

FIGURE 39: 3D $2^{\text {nd }}$ order MGRF neighborhood system. Note that the reference voxel is shown in red and $a$ represents the absolute distance between two voxels in the same and adjacent MRI slices, or cross-sections

the conditional image intensity model $P(\mathbf{g} \mid \mathbf{m})$, to build an initial region map. The final Bayesian segmentation is performed using the identified joint MGRF model of the DWI data and its region maps. Finally, the proposed prostate segmentation approach involves the steps summarized in Algorithm V.B.1.

\section{Nonrigid Registration}

The nonrigid registration of the DWI data of different b-values is performed by solving the second-order linear partial differential Laplace equation as described in Section IV.B.2. For completeness, the main steps of the Laplace-based nonrigid registration is summarized in Figure 40 as follows:

1. Generation of the distance maps inside the prostate regions (Figure $40(a, b)$ ). 


\section{$\overline{\text { Algorithm } 4 \text { Key Steps for prostate Segmentation }}$}

1. Input: The 3D DWI prostate data $g$ to be segmented.

2. Construct the shape prior from the training data.

3. Approximate the marginal intensity distribution by the linear combinations of discrete Gaussians (LCDG) with two dominant modes.

4. Form an initial region map $m$ using the estimated LCDG model.

5. Find the Gibbs potentials for the Markov-Gibbs random field (MGRF) model from the initial map.

6. Improve the region map using voxel-wise stochastic relaxation (Iterative conditional mode-ICM [107]) through successive iterations to maximize the log likelihood function of Equation (9) until the log likelihood remains almost the same for two successive iterations.

7. Output: The 3D prostate segmentation is the final estimate region map, $\mathbf{m}$.

2. Generation of the iso-contours using distance maps in step 1 in (Figure $40(\mathrm{c}, \mathrm{d})$ ).

3. Solution of the Laplace equation between respective reference and target isocontours to co-allocate the corresponding points.

\section{Diffusion Characterization and Tumor Boundary Determination}

To characterize the physiological data, color-coded maps that illustrate the propagation o of diffusion in the prostate tissues are constructed. To construct the initial color maps, we have to estimate the changes in image signals $\delta_{x, y, z}$ due to the Brownian motion. These changes are estimated from the constructed normalized diffusion as the difference between the signals of image sequences at two differ- 


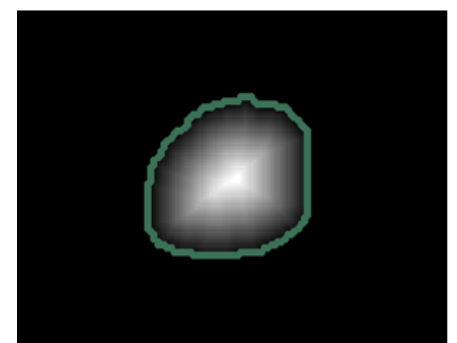

(a)

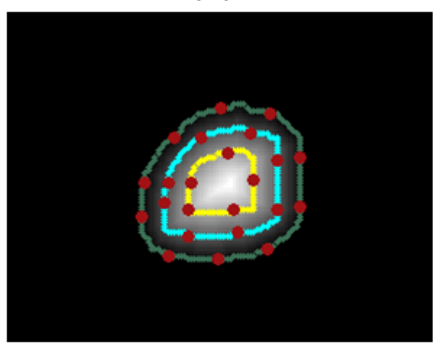

(c)

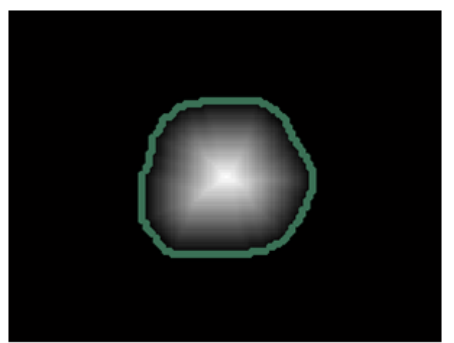

(b)

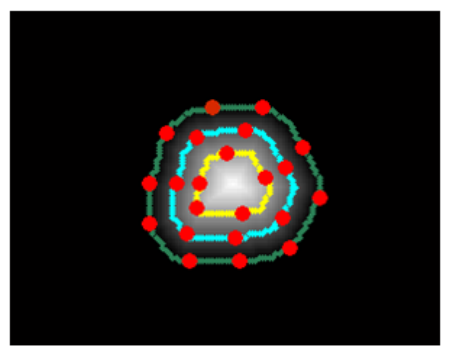

(d)

FIGURE 40: Illustration of the iso-contours generation: the reference and target distance maps $(a, b)$, and their iso-contours $(c, d)$.

ent $b$-values. DWI is performed with at least two $b$-values, including a $b$-value of $0 \mathrm{~s} / \mathrm{mm}^{2}$ and a higher $b$-value of $500-1000 \mathrm{~s} / \mathrm{mm}^{2}$ depending on the body region or organ being imaged [118]. At $b=0 \mathrm{~s} / \mathrm{mm}^{2}$, there is no diffusion sensitizing gradient with free water molecules have high signal intensity. We used $b=800 \mathrm{~s} / \mathrm{mm}^{2}$ because imaging of solid organs requires high $b$-value specially in prostate and using high $b$-values allows differentiation of areas of restricted from the normal high signal at the peripheral zone. During our trials we found the $b=800 \mathrm{~s} / \mathrm{mm}^{2}$ allows lesions differentiation with least degradation of image quality as the image quality decrease with the high $b$-values. To preserve continuity (remove inconsistencies), the initial estimated $\delta_{x, y, z}$ values are considered as samples from a Generalized Gauss-Markov Random Field (GGMRF) image model [112] of measurements with the 26-voxel neighborhood . Continuity of the constructed 3-D volume is amplified by using their MAP estimates [92]: 


$$
\begin{aligned}
\widehat{\delta}_{x, y, z}= & \arg \min _{\widetilde{\delta}_{x, y, z}}\left\{\left|\delta_{x, y, z}-\widetilde{\delta}_{x, y, z}\right|^{\alpha}+\rho^{\alpha} \lambda^{\beta}\right. \\
& \left.\sum_{\left(x^{\prime}, y^{\prime}, z^{\prime}\right) \in \nu_{(x, y, z)}} \eta_{(x, y, z),\left(x^{\prime}, y^{\prime}, z^{\prime}\right)}\left|\widetilde{\delta}_{x, y, z}-\delta_{x^{\prime}, y^{\prime}, z^{\prime}}\right|^{\beta}\right\}
\end{aligned}
$$

where $\delta_{x, y, z}$ and $\widetilde{\delta}_{x, y, z}$ denote the original values and their expected estimates, $\nu_{(x, y, z)}$ is the 26-neighborhood voxel set, $\eta_{., .}$is the GGMRF potential, and $\rho$ and $\lambda$ are scaling factors (Figure 41. The parameter $\beta \in[1.01,2.0]$ controls the level of smoothing (e.g., smooth, $\beta=2$, vs. relatively abrupt edges, $\beta=1.01$ ). The parameter $\alpha \in\{1,2\}$ determines the Gaussian, $\alpha=2$, or Laplace, $\alpha=1$, prior distribution of the estimator. Then, the color maps are generated based on the final estimated $\widehat{\delta}$ (see Figure 42).
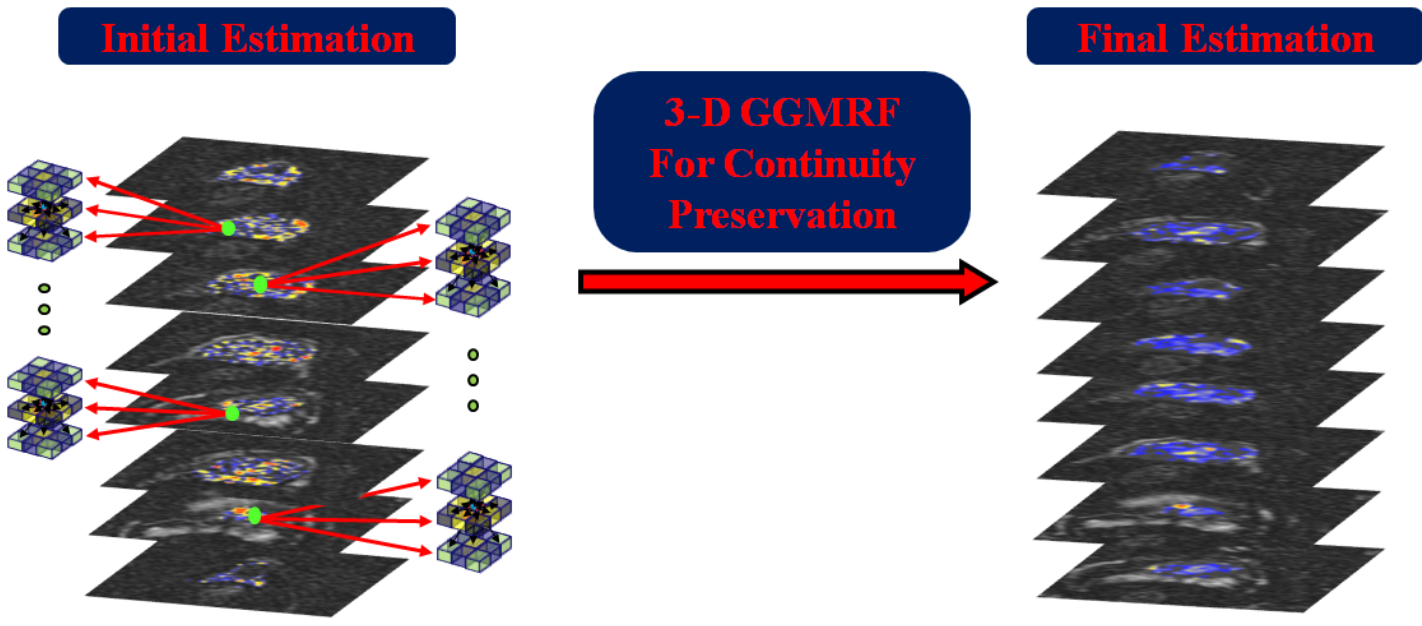

FIGURE 41: Enhanced perfusion estimation and continuity analysis using the 3D GGMRF image model.

Finally, locate the boundary of the detected tumors (see Figure 43), which is important to determine the cancer stage in case of malignancy, we used a level set-based deformable model controlled by a stochastic speed function [119]. The 


\section{Color Scale}

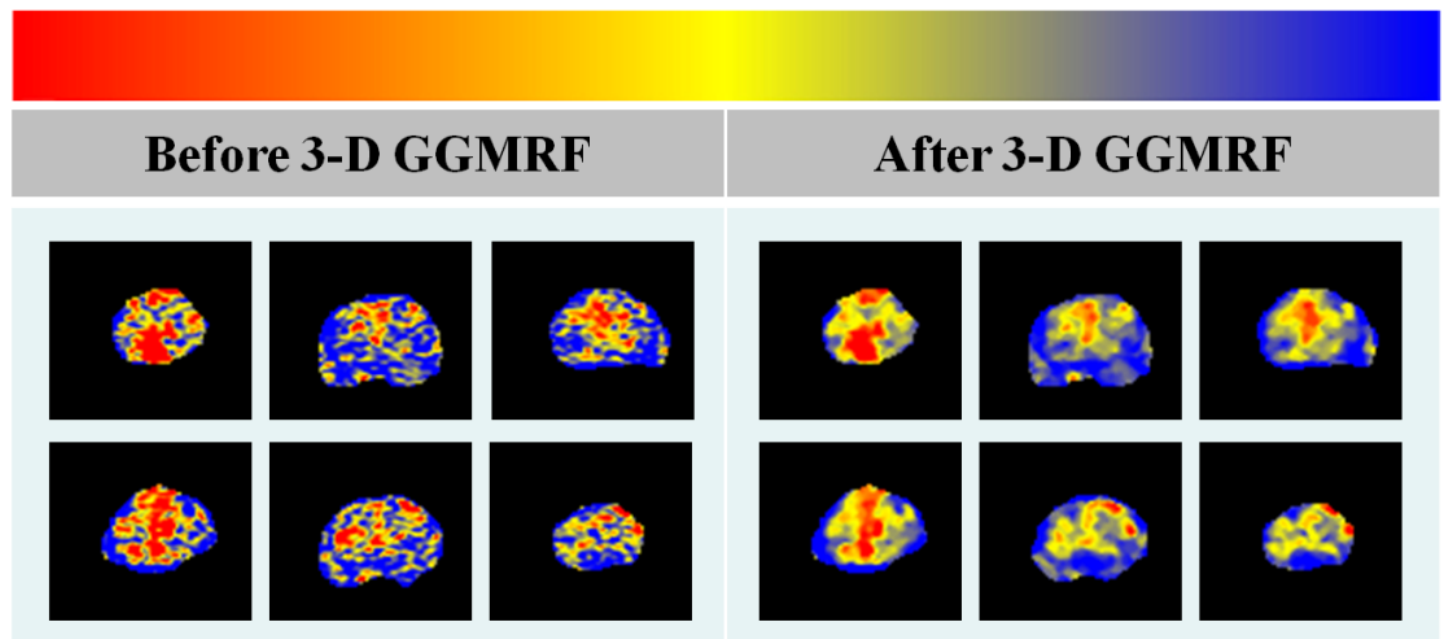

FIGURE 42: Color-coded maps for three of the test subjects (column wise) before and after the 3-D GGMRF smoothing. The red and blue ends of the color scale relate to the maximum and minimum changes, respectively.

latter accounts for the diffusion information and spatial interactions between the prostate voxels. 

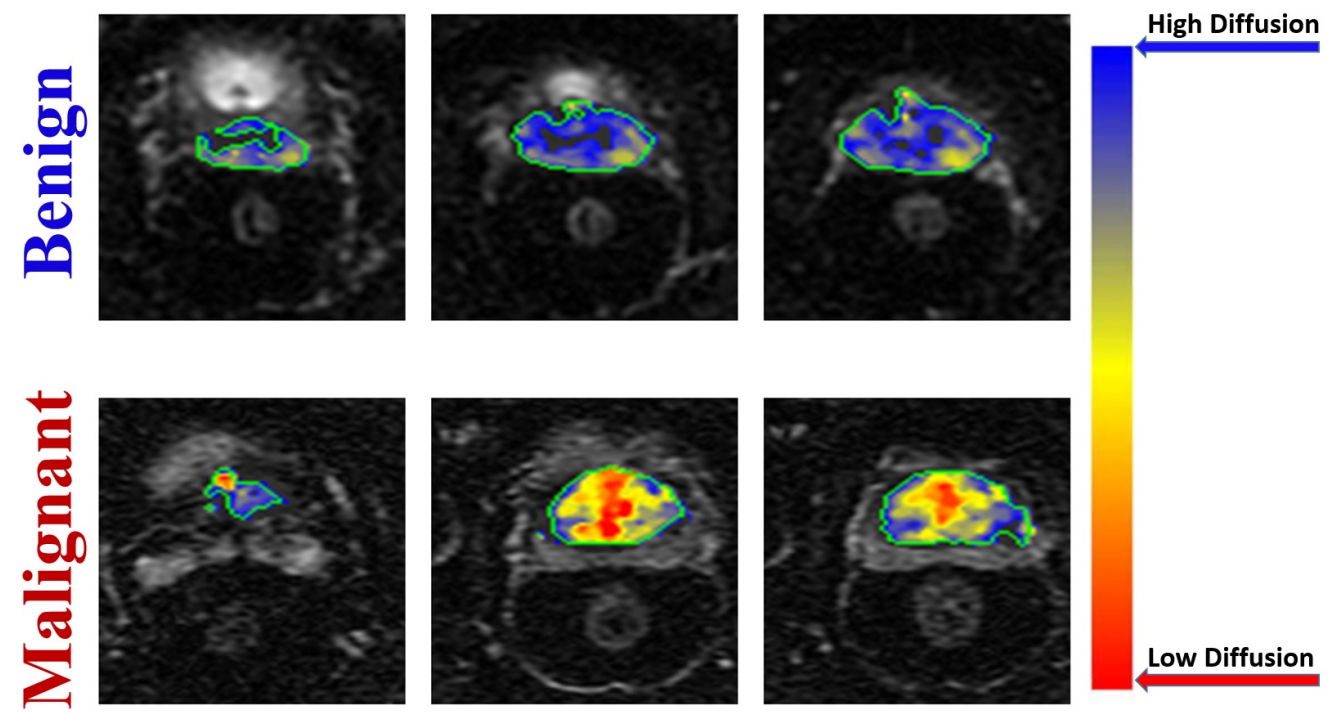

Low Diffusion

FIGURE 43: Pixel-wise parametric map display of the diffusion information and the spatial interactions between the prostate voxels. The red and blue hues of each color scale correspond to enhanced and reduced perfusion, respectively.

\section{EXPERIMENTAL RESULTS}

1. Patients and Data Acquisition

The performance of the proposed framework has been evaluated by applying it on DWI prostate images collected from 30 patients. These patients had biopsy-proven prostate cancer and underwent DWI at 1.5 T (SIGNA Horizon, General Electric Medical Systems, Milwaukee, WI). a DWI was then obtained using mono-directional gradients and a multi-section Fast Spin Echo type (FSE) echoplanar sequence in the axial plane using a body coil with the following imaging parameters: $T_{E}: 84: 6 m s ; T_{R}: 8.000 m s$; Band Width 142 kilohertz (kHz); field-ofview (FOV) was $34 \mathrm{~cm}$; slice thickness was $3 \mathrm{~mm}$; inter-slice gap $0 \mathrm{~mm}$; seven excitations, water excitation with $b$-value of $0 \mathrm{~s} / \mathrm{mm}^{2}$ and $800 \mathrm{~s} / \mathrm{mm}^{2}$. Fifty-four slices 
TABLE 6: Error Statistics over all test data sets.

\begin{tabular}{|c|c|c|c|}
\cline { 2 - 4 } \multicolumn{1}{c|}{} & PPV & SEN & DSC \\
\hline Mean \pm SD & $0.952 \pm 0.004$ & $0.816 \pm 0.004$ & $0.991 \pm 0.004$ \\
\hline
\end{tabular}

were obtained in 120 seconds to cover the prostate in each patient. Note that all the subjects were diagnosed using a biopsy (ground truth).

\section{Segmentation Results}

The proposed segmentation approach has been tested on DWI sequences for 30 independent subjects. Figure 44 shows some segmentation results of the prostate region at selected image sections for different subjects and their associated false positive (FP) and false negative (FN) segmentation errors, with respect to the ground truth segmentation. The ground truths were obtained by manual delineation of the prostate borders by an MR imaging expert. The positive predictive value (PPV), sensitivity (SEN), and Dice similarity coefficient (DSC) statistics for the proposed approach are summarized in Table 6 . To highlight the advantage of the proposed segmentation approach, all time series images have been segmented using the shape-based (SB) approach proposed by Tsai et al. [2]. The comparative results for a few of them are shown in Figure 45 and Table 7 summarizes the segmentation error statistics of the proposed approach and the SB approach with respect to ground truth. The differences between the mean errors of the proposed approach and the SB approach are shown to be statistically significant by the unpaired t-test (the two-tailed value $P$ is less than 0.0001 ). 

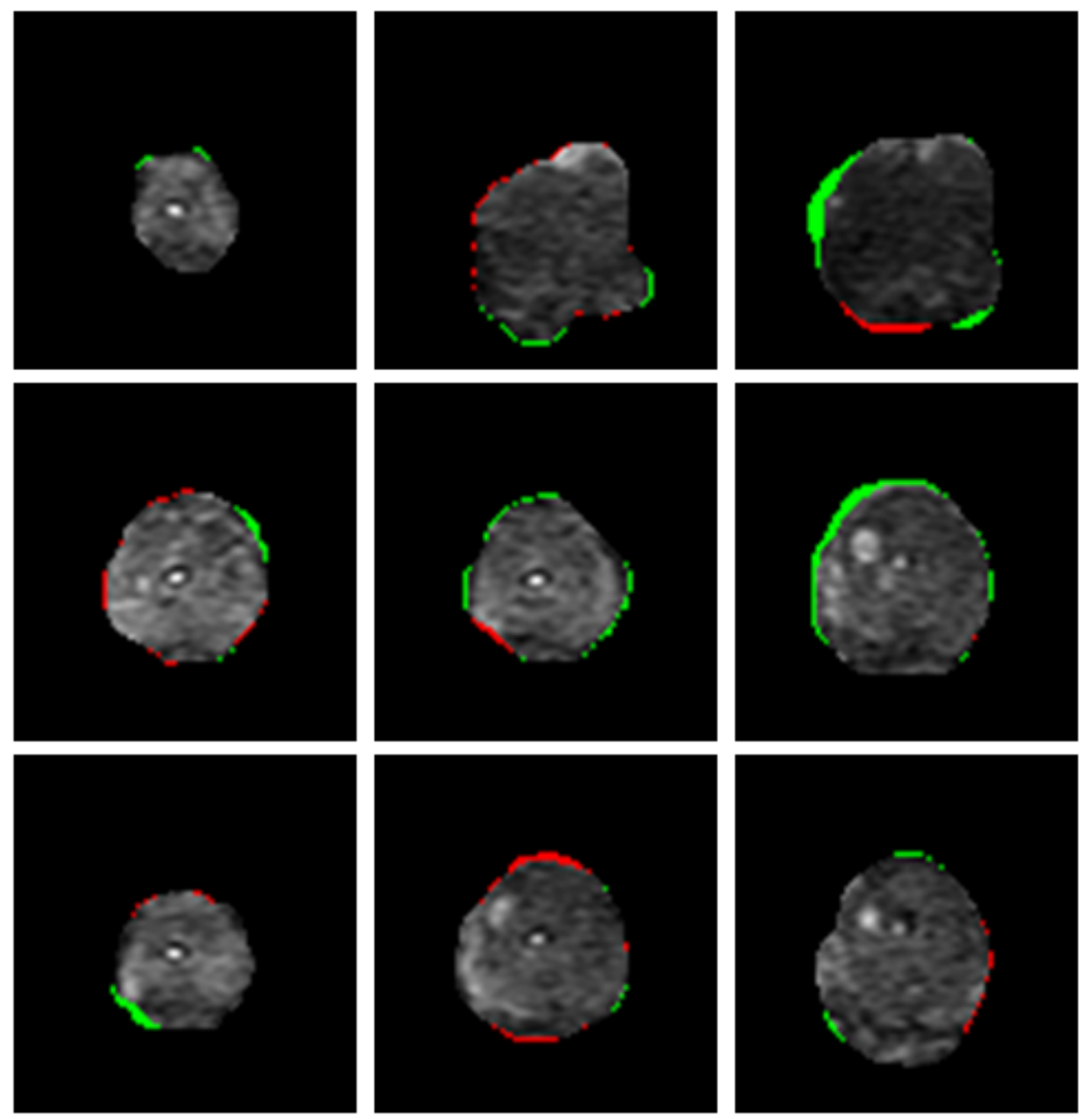

FIGURE 44: Segmentation result and their associated FN (green) and FP (red) errors referenced to the ground truth $G$.

3. Diagnostic Results

The ultimate goal of the proposed framework is to distinguish between benign and malignant detected tumors. The malignant tissues show higher signal intensity with a $b$-value of $800 \mathrm{~s} / \mathrm{mm}^{2}$, and a lower Apparent Diffusion Coefficient (ADC) compared with benign and normal tissue due to the replacement of normal tissue. To distinguish between the benign and malignant cases, we used a $k_{n}$-Nearest Neighbor classifier to learn statistical characteristics of the DWI. The characteristics are obtained from the training sets containing both benign and malignant cases. After training, three features, which are the mean intensity value of the DWI at $0 \mathrm{~s} / \mathrm{mm}^{2}$, the mean intensity value of the DWI at $800 \mathrm{~s} / \mathrm{mm}^{2}$, and the 
TABLE 7: A Comparative segmentation accuracy over all test data sets for our approach and [2]. Note that STD stands for standard deviation.

\begin{tabular}{|c|c|c|}
\hline \multicolumn{2}{|c|}{$E_{\text {avg }} \%$} & \multirow[b]{2}{*}[2]{} \\
\hline & Our approach & \\
\hline Min. Error\% & 0 & 0 \\
\hline Max. Error\% & 1.6005 & 2.7724 \\
\hline Average Error\% & 0.5500 & 1.4675 \\
\hline STD \% & 0.3085 & 0.7687 \\
\hline P-value & & 0.001 \\
\hline
\end{tabular}

TABLE 8: Area under the ROC curve for training subjects, testing subjects, and combined (training and testing subjects).

\begin{tabular}{cc}
\hline \multicolumn{2}{c}{ Area Under ROC Curve } \\
\hline Training Subjects & $A_{z}=0.996$ \\
Test Subjects & $A_{z}=0.964$ \\
All Subjects & $A_{z}=0.985$ \\
\hline
\end{tabular}

mean value of ADC maps [120], were chosen to classify the test cases. To build the $k_{n}$ classifier that characterizes the prostate tissue, we used 20 subjects for training, and the other 10 subjects for testing.

Overall system performance is demonstrated in Figure 46. For each of these ROC curves, performance is measured in comparison with the classification produced by the biopsy. Table 8 lists the area under the Receiver operating characteristic (ROC) curve performance for the ROC curves shown in Figure 46.

For regional display, we explored pixel-by-pixel maps of the registered dif- 
(a)
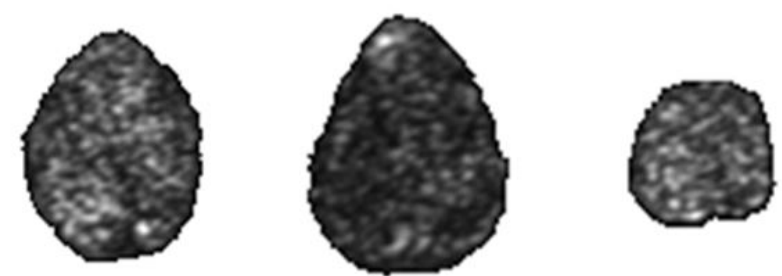

(b)
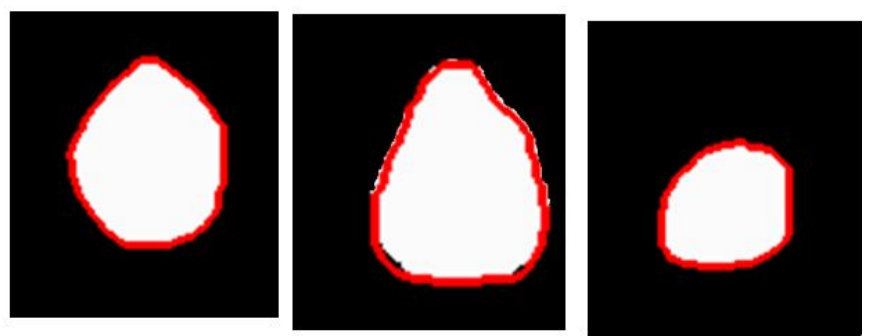

(c)
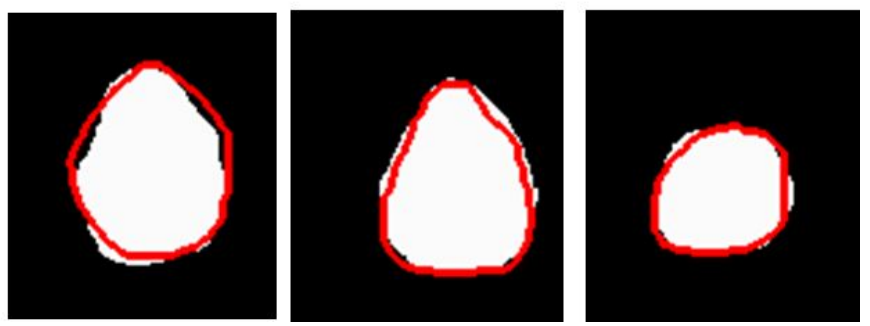

FIGURE 45: 3-D prostate segmentation projected onto 2-D. (a) 2-D visualization for our segmented prostates for three of the test subjects, (b) our segmentation (red) in comparison with the ground truth (white), and (c) the segmentation with the algorithm in [2] (red) comparison with the ground truth.

fusion data. The diffusion was computed for each pixel and superimposed on an image slice to form a parametric image. Also, for visual assessment of the prostate tumor, the tumor contours were determined. Figure 47 (c) shows the diffusion map for selected image sections for four subjects involved in our study. 


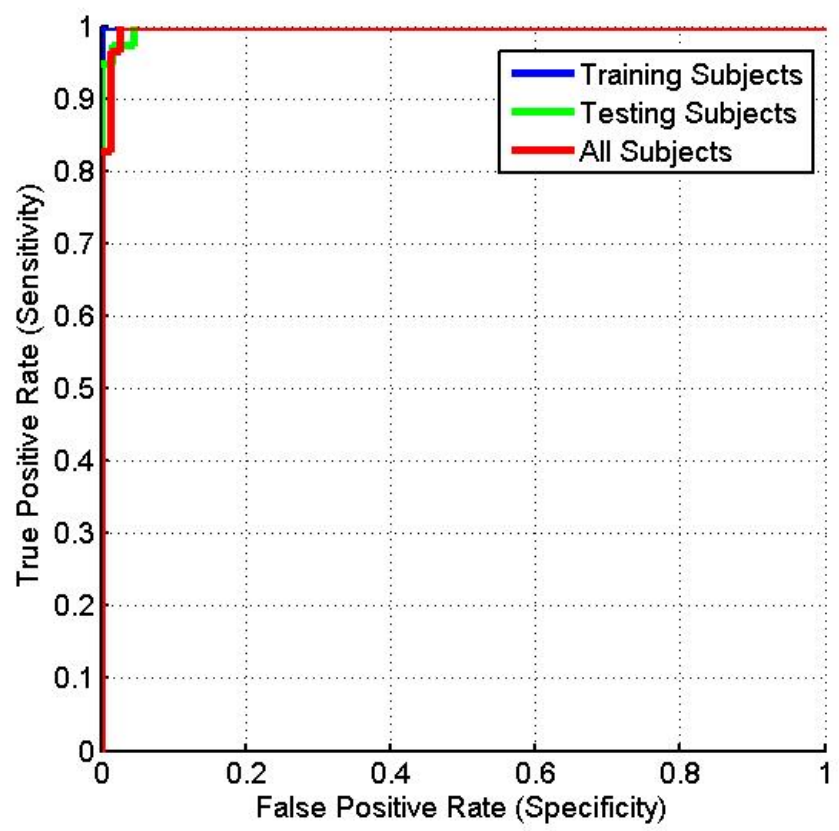

FIGURE 46: Receiver operating characteristic curves for training subjects, testing subjects, and combined (training and testing subjects). CAD performance is with respect to classification produced by the expert. 


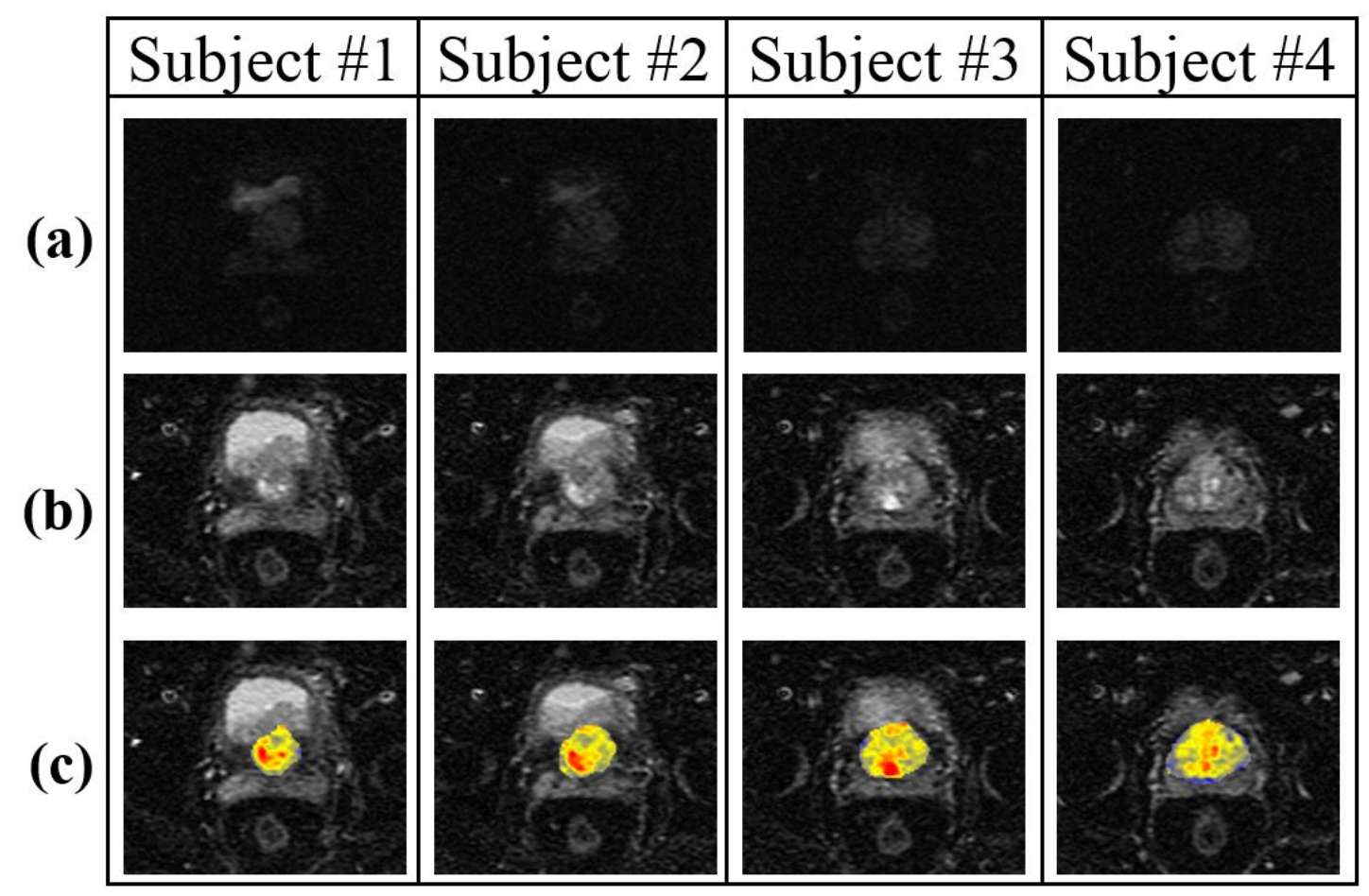

FIGURE 47: Diffusion MR images for the prostate at (a) b-value of $0 \mathrm{~s} / \mathrm{mm}^{2}$, (b) b value of $800 \mathrm{~s} / \mathrm{mm}^{2}$, and (c) tumor progression for four of the test subjects. 


\section{SUMMARY}

In this chapter, we presented a novel fully automatic framework for detecting prostate cancer using DWI. The framework includes prostate segmentation, nonrigid registration, and KNN-based classification. For prostate segmentation, we introduced a new 3D approach that is based on a MAP estimate of a new loglikelihood function that accounts for the shape priori, the spatial interaction, and the current appearance of the prostate and its background which increases the accuracy of automatic segmentation, evidenced by the error and the DSC analysis (Tables 7 and 6). Following segmentation, we used a nonrigid registration approach that deforms the prostate object on iso-contours instead of a square lattice, which provides higher degrees of freedom to obtain accurate deformation. In the classification step, the segmented prostate regions are classified into malignant or benign using the $k_{n}$ classifier. Applications of the proposed framework can assist the radiologist in detecting all prostate cancer locations and could replace the use of current technologies to determine the type of prostate cancer. The work presented in this chapter has been published in the IEEE International Conference on Image Processing (ICIP), [42], Journal of Biomedical Science and Engineering [33], and the Biomedical Science and Engineering Conference (BSEC) [29]. 


\section{CHAPTER VI}

\section{CONCLUSIONS AND FUTURE WORK}

The work presented in this dissertation documents the ability of DCE-MRI and DWI to distinguish between the benign and malignant prostate tumor cases. The key main advantage of the proposed computer-aided diagnostic (CAD) system for distinguishing between the benign and malignant cases is the ability of the developed motion correction model to provide accurate pixel-on-pixel matches of the registered images for generating and displaying parametric maps. These capabilities are of great importance for the radiologists to help investigate, in the case of cancer, in which local regions need attention and follow-up with appropriate treatment. We give a summary of the main contributions of this dissertation is as follows:

- A novel 3D prostate segmentation approach is proposed. The proposed approach is based on a Maximum a Posteriori (MAP) estimate of a log-likelihood function that accounts for three image features: the higher-order spatialinteraction between the image pixels, prior probabilistic shape model, and first-order visual appearance of the prostate. These three features are integrated into a two level joint Markov-Gibbs random field (MGRF) model of the prostate and its background. As demonstrated in the experimental results, the DSC for extracting the prostate is $93.34 \pm 0.004 \%$, which confirms the high accuracy of the proposed prostate segmentation approach.

- An unsupervised probabilistic model to learn the second- and higher-order 
spatial interactions between the object pixels (voxels) from medical images is proposed. The model possesses the ability to account for the appearance features to learn the inhomogeneity in the prostate. The proposed model adds to the pairwise the higher-order spatial interactions between region labels of a given segmentation.

- An adaptive probabilistic shape model that has the ability to learn both the shape of the prostate and the subject-to-subject variability is proposed. The adaptive shape model has been successfully used to guide the classification of prostate cancer and has shown an ability to account for the complexity of the prostate shape. The results of this model confirm its benefits and encourage using it to model other medical structures.

- A nonrigid registration to compensate for local object deformations caused by physiological effects is proposed. The proposed nonrigid registration deforms each pixel of the target object over a set of nested, equi-spaced contours (i.e., iso-contours) to closely match the reference object, by exploiting the geometric features, rather than image intensities to avoid problems associated with nonlinear intensity variations in the prostate.

Several possibilities for the future work of this dissertation include, but are not limited to, the following:

- Investigating the fusion between DCE-MRI and DWI. This fusion is expected to give better diagnosis results of the prostate cancer.

- Investigating the integration of the proposed work with the BioImaging lab work for the detection of brain disorders such as autism [121-152] and dyslexia [153-165].

- Testing the proposed mathematical models and learning techniques in other 
clinical studies, such as detection of lung cancer [166-207] , early detection of acute renal rejection [113, 208-230], and cardiac MRI [111, 119, 231-245] 


\section{REFERENCES}

[1] American Cancer Society. Global Cancer Facts and Figures 2014. National Home Office: American Cancer Society Inc, 2014.

[2] A. Tsai, Jr. Yezzi, A., W. Wells, C. Tempany, D. Tucker, A. Fan, W.E. Grimson, and A. Willsky. A shape-based approach to the segmentation of medical imagery using level sets. Medical Imaging, IEEE Transactions on, 22(2):137154, Feb 2003.

[3] J. G. E. Awad. Prostate Segmentation and Regions of Interest Detection in Transrectal Ultrasound Images. PhD thesis, University of Waterloo, 2007.

[4] J. E. McNeal. The zonal anatomy of the prostate. Prostate, 2(1):35-49, 1981.

[5] B. Trkbey, D. Thomasson, Y. Pang, M. Bernardo, and P. L. Choyke. The role of dynamic contrast-enhanced mri in cancer diagnosis and treatment. Turkish Society of Radiology, 16:186-192, 2010.

[6] L. G. Gomella and F. Allen. Treatment Choices for Men Living with Advanced Prostate Cancer. cancercare, CancerCare, New York, NY, 2006.

[7] D. T. S. Chang, B. Challacombe, and N. Lawrentschuk. Transperineal biopsy of the prostate-is this the future? Nat Rev Urol, 10(12):690-702, 2013.

[8] S. Klein, M. Staring, and J.P.W. Pluim. Evaluation of optimization methods for nonrigid medical image registration using mutual information and bsplines. Image Processing, IEEE Transactions on, 16(12):2879-2890, Dec 2007.

[9] American Cancer Society. Cancer Facts and Figures 2014. National Home Office: American Cancer Society Inc, 2014.

[10] E.David Crawford. Epidemiology of prostate cancer. Urology, 62(6, Supplement 1):3 - 12, 2003.

[11] A. Jemal, R. Siegel, E. Ward, T. Murray, J. Xu, and M. Thun. Cancer statistics 2007. Cancer Journal for Clinicians, 57:43-66, 2007.

[12] W. J. Catalona, J. P. Richie, F. R. Ahmann, M. A. Hudson, P. T. Scardino, R. C. Flanigan, J. B. deKernion, T. L. Ratliff, L. R. Kavoussi, B. L. Dalkin, and et al. Comparison of digital rectal examination and serum prostate specific antigen in the early detection of prostate cancer: results of a multicenter clinical trial of 6,630 men. The Journal of urology, 151(5):1283-90, 1994. 
[13] M. B. Matthew, R. C.and Jeannette, W. K. Philip, and R. C. Peter. Contemporary trends in low risk prostate cancer: Risk assessment and treatment. The Journal of Urology, 178(3, Supplement):S14 - S19, 2007.

[14] A. S. Jr. Joseph, T. S. Peter, I. R. Martin, D. H. Alberto, C. R. Steven, and J. E. Marlene. Transrectal ultrasound versus digital rectal examination for the staging of carcinoma of the prostate: Results of a prospective, multiinstitutional trial. The Journal of Urology, 157(3):902 - 906, 1997.

[15] B. Turner, Ph. Aslet, L. Drudge-Coates, H. Forristal, L. Gruschy, S. Hieronymi, K. Mowle, M. Pietrasik, and A. Vis. Transrectal ultrasound guided biopsy of the prostate. European Association of Urology Nurses, 2011.

[16] J. E. Langer. The current role of transrectal ultrasonography in the evaluation of prostate carcinoma. Seminars in Roentgenology, 34(4):284-294, 1999. Imaging of the Prostate.

[17] J. T. Wei. Limitations of a contemporary prostate biopsy: The blind march forward. Urologic Oncology: Seminars and Original Investigations, 28(5):546 $549,2010$.

[18] A. V. Taira, G. S. Merrick, R. W. Galbreath, H. Andreini, W. Taubenslag, R. Curtis, W. M. Butler, E. Adamovich, and K. E. Wallner. Performance of transperineal template-guided mapping biopsy in detecting prostate cancer in the initial and repeat biopsy setting. Prostate Cancer Prostatic Dis, 13(1):7177, 2010.

[19] A. G. Webb. Introduction to Biomedical Imaging. Wiley-IEEE Press, NJ, USA, 2003.

[20] T. M. Blodgett, C. C. Meltzer, and D. W. Townsend. Pet/ct: Form and function. Radiology, 242:360-385, 2007.

[21] D. Shen, Y. Zhan, and C. Davatzikos. Segmentation of prostate boundaries from ultrasound images using statistical shape model. IEEE Transactions on Medical Imaging, 22:539-551, 2003.

[22] H. M. Ladak, F. Mao, Y. Wang, D. B. Downey, D. A. Steinman, and A. Fenster. Prostate boundary segmentation from $2 \mathrm{~d}$ ultrasound images. Medical physics, 27:1777-1788, 2000.

[23] G. Pareek, U. R. Acharya, S. V. Sree, G. Swapna, R. Yantri, R. J. Martis, L. Saba, G. Krishnamurthi, G. Mallarini, A. El-Baz, S. Al Ekish, M. Beland, and J. S. Suri. Prostate tissue characterization/classification in 144 patient population using wavelet and higher order spectra features from transrectal ultrasound images. Technol Cancer Res Treat, 12:545-57, 2013.

[24] R. Zwiggelaar, Y. Zhu, and S. Williams. Semi-automatic segmentation of the prostate. In Pattern Recognition and Image Analysis, volume 2652 of Lecture Notes in Computer Science, pages 1108-1116. Springer Berlin Heidelberg, 2003. 
[25] E. M. Haacke, R. W. Brown, M. R. Thompson, and R. Venkatesan. Magnetic Resonance Imaging: Physical Principles and Sequence Design. Wiley-Liss, New York, USA, 1st edition, 1999.

[26] Y. J. Choi, J. K. Kim, N. Kim, K. W. Kim, E. K. Choi, and K. Cho. Functional mr imaging of prostate cancer. RadioGraphics, 27(1):63-75, 2007. PMID: 17234999.

[27] J. K. Kim, S. S. Hong, Y. J. Choi, S. H. Park, H. Ahn, C. Kim, and K. Cho. Wash-in rate on the basis of dynamic contrast-enhanced mri: Usefulness for prostate cancer detection and localization. Journal of Magnetic Resonance Imaging, 22(5):639-646, 2005.

[28] C. Sato, S. Naganawa, T. Nakamura, H. Kumada, S. Miura, O Takizawa, and T. Ishigaki. Differentiation of noncancerous tissue and cancer lesions by apparent diffusion coefficient values in transition and peripheral zones of the prostate. Journal of Magnetic Resonance Imaging, 21(3):258-262, 2005.

[29] A. Firjani, A. Elmaghraby, and A. El-Baz. Mri-based diagnostic system for early detection of prostate cancer. In Biomedical Sciences and Engineering Conference (BSEC), 2013, pages 1-4, May 2013.

[30] A. Firjani, F. Khalifa, A. Elnakib, G. Gimel'farb, M. El-Ghar, A. Elmaghraby, and A. El-Baz. A novel image-based approach for early detection of prostate cancer using dce-mri. In Kenji Suzuki, editor, Computational Intelligence in Biomedical Imaging, pages 55-82. Springer New York, 2014.

[31] A. Firjani, F. Khalifa, A. Elnakib, G. Gimel'farb, M. Abo El-Ghar, A. Elmaghraby, and A. El-Baz. Non-invasive image-based approach for early detection of prostate cancer. In Proceedings of Fourth International Conference on Developments in eSystems Engineering, (DeSE'11), pages 172-177, Dubai, UAE, December 6-8, 2011.

[32] A. Firjani, A. Elnakib, F. Khalifa, A. El-Baz, G. Gimel'farb, M. Abou El-Ghar, and A. Elmaghraby. A novel 3D segmentation approach for segmenting the prostate from dynamic contrast enhanced MRI using current appearance and learned shape prior. In Proceedings of IEEE International Symposium on Signal Processing and Information Technology, (ISSPIT'10), pages 137-143, Luxor, Egypt, December 15-18, 2010.

[33] A. Firjani, A. Elnakib, F. Khalifa, G. Gimelfarb, M. El-Ghar, A. Elmaghraby, and A. El-Baz. A diffusion-weighted imaging based diagnostic system for early detection of prostate cancer. Journal of Biomedical Science and Engineering, 6(3):346-356, 2013.

[34] R. Shimofusa, H. Fujimoto, H. Akamata, K. Motoori, S. Yamamoto, T. Ueda, and H. Ito. Diffusion-weighted imaging of prostate cancer. J Comput Assist Tomogr , 29(2):149-53, 2005. 
[35] Y. Ueno, S. Takahashi, K. Kitajima, T. Kimura, I. Aoki, F. Kawakami, H. Miyake, Y. Ohno, and K. Sugimura. Computed diffusion-weighted imaging using 3-t magnetic resonance imaging for prostate cancer diagnosis. European Radiology, 23(12):3509-3516, 2013.

[36] K. Yoshimitsu, K. Kiyoshima, H. Irie, T. Tajima, Y. Asayama, M. Hirakawa, K. Ishigami, S. Naito, and H. Honda. Usefulness of apparent diffusion coefficient map in diagnosing prostate carcinoma: Correlation with stepwise histopathology. Journal of Magnetic Resonance Imaging, 27(1):132-139, 2008.

[37] C. K. Kim, B. K. Park, and H. M. Lee. Prediction of locally recurrent prostate cancer after radiation therapy: Incremental value of 3t diffusion-weighted mri. Journal of Magnetic Resonance Imaging, 29(2):391-397, 2009.

[38] H. K. Lim, J. K. Kim, K. Ah Kim, and K. Cho. Prostate cancer: Apparent diffusion coefficient map with $\mathrm{t} 2$-weighted images for detectiona multireader study. Radiology, 250(1):145-151, 2009.

[39] T. Hacklnder, C. Scharwchter, R. Golz, and H. Mertens. Value of diffusionweighted imaging for diagnosing vertebral metastases due to prostate cancer in comparison to other primary tumors. Fortschr Rntgenstr, 04:214-424, 2006.

[40] K. K Yu and H Hricak. Imaging prostate cancer. Radiol Clin North Am, 38(1):59-85, 2000.

[41] M. A. Haider, T. H. van der Kwast, J. Tanguay, A. J. Evans, A. T. Hashmi, G. Lockwood, and J. Trachtenberg. Combined t2-weighted and diffusionweighted mri for localization of prostate cancer. American Journal of Roentgenology, 189(2):323-328, 2007.

[42] A. Firjani, F. Khalifa, A. Elnakib, G. Gimel'farb, A. Elmaghraby, and A. ElBaz. A novel image-based approach for early detection of prostate cancer. In Proceedings of IEEE International Conference on Image Processing, (ICIP'12), pages 2849-2852, Lake Buena Vista, Florida, September 30-October 3, 2012.

[43] A. Firjani, A. Elnakib, F. Khalifa, G. Gimel'farb, M. Abo El-Ghar, A. Elmaghraby, and A. El-Baz. A new 3D automatic segmentation framework for accurate extraction of prostate from diffusion imaging. In Proceedings of Biomedical Science and Engineering Conference-Image Informatics and Analytics in Biomedicine, (BSEC'11), pages 1306-1309, Knoxville, Tennessee, March 1517, 2011.

[44] P.D. Allen, J. Graham, D.C. Williamson, and C.E. Hutchinson. Differential segmentation of the prostate in mr images using combined $3 \mathrm{~d}$ shape modelling and voxel classification. In Biomedical Imaging: Nano to Macro, 2006. 3rd IEEE International Symposium on, pages 410-413, April 2006.

[45] Y. Zhu, S. Williams, and R. Zwiggelaar. Segmentation of volumetric prostate mri datausing hybrid $2 \mathrm{~d}+3 \mathrm{~d}$ shape modeling. Medical Image Understanding and Analysis, pages 61-64, 2004. 
[46] S. Klein, U. A. van der Heidi, B. W. Raaymakers, A. Kotte, M. Staring, and J. Pluim. Segmentation of the prostate in $\mathrm{mr}$ images by atlas matching. Biomedical Imaging: From Nano to Macro, pages 1300-1303, 2007.

[47] D. Flores-Tapia, N. Venugopal, G. Thomas, B. McCurdy, L. Ryner, and S. Pistorius. Real time mri prostate segmentation based on wavelet multiscale products flow tracking. In Engineering in Medicine and Biology Society (EMBC), 2010 Annual International Conference of the IEEE, pages 5034-5037, Aug 2010.

[48] S. Mallat and S. Zhong. Characterization of signals from multiscale edges. Pattern Analysis and Machine Intelligence, IEEE Transactions on, 14(7):710-732, Jul 1992.

[49] R. Toth, P. Tiwari, M. Rosen, A. Kalyanpur, S. Pungavkar, and A. Madabhushi. A multi-modal prostate segmentation scheme by combining spectral clustering and active shape models. Medical Imaging 2008, 6914:69144S69144S, 2008.

[50] S.Vikal, S. Haker, C. Tempany, and G. Fichtinger. Prostate contouring in mri guided biopsy. In SPIE, volume 7259, 2009.

[51] A. Firjany, A. Elnakib, A. El-Baz, G. Gimel'farb, M. El-Ghar, and A. Elmagharby. Novel stochastic framework for accurate segmentation of prostate in dynamic contrast enhanced mri. In A. Madabhushi, J. Dowling, P. Yan, A. Fenster, P. Abolmaesumi, and N. Hata, editors, Prostate Cancer Imaging. Computer-Aided Diagnosis, Prognosis, and Intervention, volume 6367 of Lecture Notes in Computer Science, pages 121-130. Springer Berlin Heidelberg, 2010.

[52] A. Firjani, A. Elnakib, F. Khalifa, G. L. Gimel'farb, M. Abou El-Ghar, J. Suri, A. Elmaghraby, and A. El-Baz. A new 3d automatic segmentation framework for accurate segmentation of prostate from dce-mri. In ISBI, pages 1476-1479, 2011.

[53] P. Liu, S. Wang, B. Turkbey, K. Grant, P. Pinto, P. Choyke, B. J. Wood, and R. M. Summers. A prostate cancer computer-aided diagnosis system using multimodal magnetic resonance imaging and targeted biopsy labels. volume 8670, pages 86701G-86701G-6, 2013.

[54] P. Liao, T. Chen, and P. Chung. A fast algorithm for multilevel thresholding. Journal of Information Science and Engineering, 17:713-727, 2001.

[55] N. Makni, P. Puech, R. Lopes, A.S. Dewalle, O. Colot, and N. Betrouni. Combining a deformable model and a probabilistic framework for an automatic $3 \mathrm{~d}$ segmentation of prostate on mri. International Journal of Computer Assisted Radiology and Surgery, 4(2):181-188, 2009.

[56] Xin Liu, D.L. Langer, M.A. Haider, Yongyi Yang, M.N. Wernick, and I.S. Yetik. Prostate cancer segmentation with simultaneous estimation of markov random field parameters and class. Medical Imaging, IEEE Transactions on, 28(6):906-915, June 2009. 
[57] Y. Artan, M.A. Haider, D.L. Langer, T.H. van der Kwast, A.J. Evans, Yongyi Yang, M.N. Wernick, J. Trachtenberg, and I.S. Yetik. Prostate cancer localization with multispectral mri using cost-sensitive support vector machines and conditional random fields. Image Processing, IEEE Transactions on, 19(9):24442455, Sept 2010.

[58] S. Ozer, M.A. Haider, D.L. Langer, T.H. van der Kwast, A.J. Evans, M.N. Wernick, J. Trachtenberg, and I.S. Yetik. Prostate cancer localization with multispectral mri based on relevance vector machines. In Biomedical Imaging: From Nano to Macro, 2009. ISBI '09. IEEE International Symposium on, pages 73-76, June 2009.

[59] M. E. Tipping. The relevance vector machine, 2000.

[60] Y. Gao, R. Sandhu, G. Fichtinger, and A.R. Tannenbaum. A coupled global registration and segmentation framework with application to magnetic resonance prostate imagery. Medical Imaging, IEEE Transactions on, 29(10):17811794 , Oct 2010.

[61] S. Martin, V. Daanenc, and J. Troccaz. Automated segmentation of the prostate in $3 \mathrm{~d} \mathrm{mr}$ images using a probabilistic atlas and a spatially constrained deformable model. Medical physics, 37:1579-1590, 2010.

[62] J. Dowling, J. Fripp, S. Chandra, J. Pluim, J. Lambert, J. Parker, J. Denham, P. Greer, and O. Salvado. Fast automatic multi-atlas segmentation of the prostate from $3 \mathrm{~d} \mathrm{mr}$ images. In Prostate Cancer Imaging. Image Analysis and Image-Guided Interventions, volume 6963 of Lecture Notes in Computer Science, pages 10-21. Springer Berlin Heidelberg, 2011.

[63] B. Rodríguez-Vila, J. Pettersson, M. Borga, F. Garcia-Vicente, E. Gomez, and H. Knutsson. 3d deformable registration for monitoring radiotherapy treatment in prostate cancer. In Image Analysis, volume 4522 of Lecture Notes in Computer Science, pages 750-759. Springer Berlin Heidelberg, 2007.

[64] S. Ghose, J. Mitra, A. Oliver, R. Marti, X. Llado, J. Freixenet, J. C. Vilanova, J. Comet, D. Sidibé, and F. Mériaudeau. A mumford-shah functional based variational model with contour, shape, and probability prior information for prostate segmentation. In IAPR International Conference on Pattern Recognition, Tsukba, Japan, November 2012.

[65] S. Ghose, A. Oliver, R. Marti, X. LladÓ, J. Freixenet, J. Mitra, J. C. Vilanova, and $\mathrm{F}$ Meriaudeau. A hybrid framework of multiple active appearance models and global registration for $3 \mathrm{~d}$ prostate segmentation in mri. volume 8314, pages 83140S-83140S-9, 2012.

[66] I. Chan, W. Wells, R. V. M. S. Haker, J. Z., K. H. Zou, S. E. Maier, and C. M. C. Tempany. Detection of prostate cancer by integration of line-scan diffusion, t2-mapping and t2-weighted magnetic resonance imaging; a multichannel statistical classifier. Medical Physics, 30:2390-2398, 2003. 
[67] A. Madabhushi, M.D. Feldman, D.N. Metaxas, J. Tomaszeweski, and D. Chute. Automated detection of prostatic adenocarcinoma from highresolution ex vivo mri. Medical Imaging, IEEE Transactions on, 24(12):16111625, Dec 2005.

[68] M.W. Engelbrecht, J.O. Barentsz, and J.G. Jager. Prostate cancer staging using imaging. Human Brain Mapping, 86:123-34, 2000.

[69] J. J. Fütterer, M. R. Engelbrecht, H. J. Huisman, G. J. Jager, C. A. Hulsbergenvan De Kaa, J. A. Witjes, and J. O. Barentsz. Staging prostate cancer with dynamic contrast-enhanced endorectal $\mathrm{mr}$ imaging prior to radical prostatectomy: Experienced versus less experienced readers. Radiology, 237(2):541$549,2005$.

[70] O. Rouvire, O. Valette, S. Grivolat, C. Colin-Pangaud, R. Bouvier, J. Y. Chapelon, A. Gelet, and D. Lyonnet. Recurrent prostate cancer after external beam radiotherapy: value of contrast-enhanced dynamic $\{\mathrm{MRI}\}$ in localizing intraprostatic tumorcorrelation with biopsy findings. Urology, 63(5):922 - 927, 2004.

[71] P. Puech, N. Betrouni, N. Makni, A. Dewalle, A. Villers, and L. Lemaitre. Computer-assisted diagnosis of prostate cancer using dce-mri data: design, implementation and preliminary results. International Journal of Computer Assisted Radiology and Surgery, 4(1):1-10, 2009.

[72] I. Ocak, M. Bernardo, G. Metzger, T. Barrett, P. Pinto, P. Albert, and P. Choyke. Dynamic contrast-enhanced mri of prostate cancer at $3 \mathrm{t}$ : A study of pharmacokinetic parameters. American Journal of Roentgenology, 189(4):W192-W201, 2007.

[73] S. Viswanath, B. Bloch, E. Genega, N. Rofsky, R. Lenkinski, J. Chappelow, R. Toth, and A. Madabhushi. A comprehensive segmentation, registration, and cancer detection scheme on 3 tesla in vivo prostate dce-mri. In D. Metaxas, L. Axel, G. Fichtinger, and G. Székely, editors, Medical Image Computing and Computer-Assisted Intervention-MICCAI 2008, volume 5241 of Lecture Notes in Computer Science, pages 662-669. Springer Berlin Heidelberg, 2008.

[74] D. de Ridder and R. P.W. Duin. Locally linear embedding for classification, 2002.

[75] P. C. Vos, T. Hambrock, J. O. Barentsz, and H. J. Huisman. Computer-assisted analysis of peripheral zone prostate lesions using t2-weighted and dynamic contrast enhanced t1-weighted mri. Physics in medicine and biology, 55(6):88899, 2010.

[76] P. C. Vos, C. A. Hambrock, T.and Hulsbergen-van de Kaa, J. J. Futterer, J. O. Barentsz, and H. J. Huisman. Computerized analysis of prostate lesions in the peripheral zone using dynamic contrast enhanced mri. Medical physics, 35(3):888-99, 2008. 
[77] D. Ampeliotis, A. Antonakoudi, K. Berberidis, E.Z. Psarakis, and A. Kounoudes. A computer-aided system for the detection of prostate cancer based on magnetic resonance image analysis. In Communications, Control and Signal Processing, 2008. ISCCSP 2008. 3rd International Symposium on, pages 1372-1377, March 2008.

[78] G. Litjens, O. Debats, J. Barentsz, N. Karssemeijer, and H. Huisman. Computer-aided detection of prostate cancer in mri. Medical Imaging, IEEE Transactions on, 33(5):1083-1092, May 2014.

[79] Y. Peng, Y. Jiang, T. Antic, M. L. Giger, S. Eggener, and A. Oto. A study of t2-weighted mr image texture features and diffusion-weighted $\mathrm{mr}$ image features for computer-aided diagnosis of prostate cancer. volume 8670, pages 86701H-86701H-6, 2013.

[80] P. Tiwari, J. Kurhanewicz, and A. Madabhushi. Multi-kernel graph embedding for detection, gleason grading of prostate cancer via mri/mrs. Medical Image Analysis, 17(2):219 - 235, 2013.

[81] A. Madabhushi, J. Shi, M. Rosen, J. E. Tomaszewski, and M. D. Feldman. Comparing classification performance of feature ensembles: Detecting prostate cancer from high resolution mri. In Computer Vision Methods in Medical Image Analysis (In conjunction with ECCV), volume 4241, pages 25-36. Springer Verlag, Springer Verlag, 2006.

[82] S. Viswanath, B. N. Bloch, M. Rosen, J. Chappelow, R. Toth, N. Rofsky, R. Lenkinski, E. Genega, A. Kalyanpur, and A. Madabhushi. Integrating structural and functional imaging for computer assisted detection of prostate cancer on multi-protocol in vivo 3 tesla mri. volume 7260, pages 72603I72603I-12, 2009.

[83] R. Lopes, A. Ayache, N. Makni, P. Puech, A. Villers, S. Mordon, and N. Betrouni. Prostate cancer characterization on $\mathrm{mr}$ images using fractal features. Medical Physics, 38(1):83-95, 2011.

[84] V. Shah, B. Turkbey, H. Mani, Y. Pang, T. Pohida, M. J. Merino, P. A. Pinto, P. L. Choyke, and M. Bernardo. Decision support system for localizing prostate cancer based on multiparametric magnetic resonance imaging. Medical Physics, 39(7):4093-4103, 2012.

[85] Y. S. Sung, H. J. Kwon, B. W. Park, G. Cho, C. K. Lee, K. S. Cho, and J. K. Kim. Prostate cancer detection on dynamic contrast-enhanced mri: computeraided diagnosis versus single perfusion parameter maps. AJR. American journal of roentgenology, 197(5):1122-9, 2011.

[86] E. Niaf, R. Flamary, O. Rouviere, C. Lartizien, and S. Canu. Kernelbased learning from both qualitative and quantitative labels: Application to prostate cancer diagnosis based on multiparametric $\mathrm{mr}$ imaging. Image Processing, IEEE Transactions on, 23(3):979-991, March 2014. 
[87] C. M. A. Hoeks, J. O. Barentsz, T. Hambrock, D. Yakar, D. M. Somford, S. W. T. P. J. Heijmink, T. W. J. Scheenen, P. C. Vos, H. Huisman, I. M. van Oort, J. A. Witjes, A. Heerschap, and J. J. Fütterer. Prostate cancer: Multiparametric $\mathrm{mr}$ imaging for detection, localization, and staging. Radiology, 261(1):46-66, 2011.

[88] D. Bonekamp, M. A. Jacobs, R. El-Khouli, D. Stoianovici, and K. J. Macura. Advancements in $\mathrm{mr}$ imaging of the prostate: From diagnosis to interventions. RadioGraphics, 31(3):677-703, 2011.

[89] G. J. Parker, J. Suckling, S. F. Tanner, A. R. Padhani, J. E. Husband, and M. O. Leach. Mriw: parametric analysis software for contrast-enhanced dynamic mr imaging in cancer. RadioGraphics, 18(2):497-506, 1998.

[90] K. Ogura, S. Maekawa, K. Okubo, Y. Aoki, T. Okada, K. Oda, Y. Watanabe, C. Tsukayama, and Y. Arai. Dynamic endorectal magnetic resonance imaging for local staging and detection of neurovascular bundle involvement of prostate cancer: correlation with histopathologic results. Urology, 57(4):721 726, 2001.

[91] A. El-Baz. Novel stochastic models for medical image analysis. PhD thesis, University of Louisville, 2006.

[92] A. El-Baz and G. Gimel'farb. Em based approximation of empirical distributions with linear combinations of discrete gaussians. In Image Processing, 2007. ICIP 2007. IEEE International Conference on, volume 4, pages 373-376, Sept 2007.

[93] A. El-Baz, A. Elnakib, F. Khalifa, M.A. El-Ghar, P. McClure, A. Soliman, and G. Gimelrfarb. Precise segmentation of 3-d magnetic resonance angiography. Biomedical Engineering, IEEE Transactions on, 59(7):2019-2029, July 2012.

[94] A. Webb. Statistical Pattern Recognition. J. Wiley \& Sons, New York, USA, 2002.

[95] D. L. Wilson and J. A. Noble. An adaptive segmentation algorithm for timeof-flight MRA data. IEEE Transaction on Medical Imaging, 18(10):938-945, 1999.

[96] A. Farag, A. El-Baz, and G. Gimel'farb. Precise segmentation of multi-modal images. IEEE Transactions on Image Processing, 15(4):952-968, 2006.

[97] A. El-Baz, A. A. Farag, and G. Gimel'farb. Iterative approximation of empirical grey-level distributions for precise segmentation of multimodal images. EURASIP Journal on Applied Signal Processing, 2005:1969-1983, 2005.

[98] A. El-Baz, A. Farag, and G. Gimel'farb. Iterative approximation of empirical grey level distributions for precise segmentation of multi-modal images. EURASIP Journal on Applied Signal Processing, 13:1969-1983, 2005. 
[99] G. L. Gimel'farb, A. A. Farag, and A. El-Baz. Expectation-maximization for a linear combination of gaussians. In Proceedings of IEEE International Conference on Pattern Recognition, (ICPR'04), pages 422-425, Cambridge, UK, August 23-26, 2004.

[100] A. Farag, A. El-Baz, and G. Gimel'farb. Density estimation using modified expectation maximization for a linear combination of gaussians. In Proceedings of IEEE International Conference on Image Processing, (ICIP'04), volume 3, pages 1871-1874, Singapore, 2004.

[101] A. A. Farag, A. El-Baz, and R. M. Mohamed. Density estimation using generalized linear model and a linear combination of gaussians. International Journal of Signal Processing, 1:76-79, 2005.

[102] A. Farag, A. El-Baz, and G. Gimel'farb. Precise image segmentation by iterative em-based approximation of empirical grey level distributions with linear combinations of Gaussians. In Computer Vision and Pattern Recognition Workshop (CVPRW'2004), pages 121-129. IEEE, 2004.

[103] A. El-Baz, R. M. Mohamed, A. A. Farag, and G. Gimel'farb. Unsupervised segmentation of multi-modal images by a precise approximation of individual modes with linear combinations of discrete gaussians. In IEEE Computer Society Conference on Computer Vision and Pattern Recognition Workshops (CVPRW'2005), pages 54-54. IEEE, 2005.

[104] A. Farag, R. Mohamed, and A. El-Baz. A unified framework for map estimation in remote sensing image segmentation. IEEE Transactions on Geoscience and Remote Sensing, pages 1617-1634, 2005.

[105] A. El-Baz, R. Mohamed, and A. Farag. Advanced support vector machines for image modeling using gibbs-markov random field. Computational Intelligence, pages 306-309, 2005.

[106] P. Viola and W. M. Wells. Alignment by maximization of mutual information. In Proc. 5th Int. Conf. Comp. Vision, pages 16-23, 1995.

[107] J. Besag. On the statistical analysis of dirty pictures. Journal of the Royal Statistical Society. Series B, 48:259-302, 1986.

[108] S. Haker, S. Angenent, A. Tannenbaum, and R. Kikinis. Nondistorting flattening maps and the 3D visualization of colon CT images. IEEE Transaction on Medical Imaging, 19(7):665-670, 2000.

[109] A.J. Yezzi, L. Zöllei, and T. Kapur. A variational framework for integrating segmentation and registration through active contours. Medical Image Analysis, 7(2):171-185, 2003.

[110] M. Prasad, A. Ramesh, P. Kavanagh, B. K. Tamarappoo, R. Nakazato, J. Gerlach, V. Cheng, L. E.J. Thomson, D. S. Berman, G. Germano, and P. J. Slomka. Quantification of $3 \mathrm{~d}$ regional myocardial wall thickening from gated magnetic resonance images. Journal of Magnetic Resonance Imaging, 31(2):317-327, 2010. 
[111] F. Khalifa, G. M. Beache, G. Gimel'farb, G. A. Giridharan, and A. El-Baz. Accurate automatic analysis of cardiac cine images. IEEE Transactions on Biomedical Engineering, 59(2):445-455, 2012.

[112] C. Bouman and K. Sauer. A generalized gaussian image model for edgepreserving map estimation. Image Processing, IEEE Transactions on, 2(3):296310, Jul 1993.

[113] F. Khalifa, A. Elnakib, G. M. Beache, G. Gimel'farb, M. A. El-Ghar, G. Sokhadze, S. Manning, P. McClure, and A. El-Baz. 3d kidney segmentation from ct images using a level set approach guided by a novel stochastic speed function. In Proceedings of International Conference on Medical Image Computing and Computer-Assisted Intervention, (MICCAI'11), pages 587-594, Toronto, Canada, Sept. 18-22, 2011.

[114] K. H. Zou, S. K. Warfield, A. Baharatha, C. Tempany, M. R. Kaus, S. J. Haker, W. M. Wells, F. A. Jolesz, and R. Kikinis. Statistical Validation of Image Segmentation Quality Based on a Spatial Overlap Index. Academic Radiology, 11:178-189, 2004.

[115] P. McClure, A. Elnakib, M. Abou El-Ghar, F. Khalifa, A. Soliman, T. El-Diasty, J. S. Suri, A. Elmaghraby, , and A. El-Baz. In-vitro and in-vivo diagnostic techniques for prostate cancer a review. Journal of Biomedical Nanotechnology, 10(2747-2777), 2014.

[116] C. Tan, J. Wang, and V. Kundra. Diffusion weighted imaging in prostate cancer. European Radiology, 21(3):593-603, 2011.

[117] Georgy Gimel'farb. Image textures and gibbs random fields. Kluwer, Dordrecht, The Netherlands, 1999.

[118] D. M. Patterson, A. R. Padhani, and D. J. Collins. Technology insight: water diffusion mri-a potential new biomarker of response to cancer therapy. Nat Clin Prac Oncol, 5(4):220-233, 2008.

[119] F. Khalifa, G. Beache, A. El-Baz, and G. Gimel'farb. Deformable model guided by stochastic speed with application in cine images segmentation. In Proceedings of IEEE International Conference on Image Processing, (ICIP'10), pages 1725-1728, Hong Kong, September 26-29, 2010.

[120] S. Walker-Samuel, M. Orton, J. K. R. Boult, and S. P. Robinson. Improving apparent diffusion coefficient estimates and elucidating tumor heterogeneity using bayesian adaptive smoothing. Magnetic Resonance in Medicine, 65(2):438-447, 2011.

[121] M. Mostapha, A. Soliman, F. Khalifa, A. Elnakib, A. Alansary, M. Nitzken, and M. F. Casanova. A statistical framework for the classification of infant $\mathrm{dt}$ images. In IEEE International Conference on Image Processing (ICIP'14), pages In-press, 2014. 
[122] A. Alansary, A. Soliman, M. Nitzken, F. Khalifa, A. Elnakib, M. F. Casanova, and A. El-Baz. An integrated geometrical and stochastic approach for accurate infant brain extraction. In IEEE International Conference on Image Processing (ICIP'14), pages In-press, 2014.

[123] J. Baruth, E. Sokhadze, A. El-Baz, G. Mathai, L. Sears, and M. F. Casanova. Transcranial Magnetic Stimulation, chapter 4, pages 143-152. Skyhorse Publishing, 2012.

[124] R. Pennington, K. Welch, E. Sokhadze, A. El-Baz, A. Farag, P. Williams, and M. Casanova. Crossing the Divide: Collaborative Efforts towards Innovative Treatments at the University of Louisville Autism Center, chapter 5, pages 161-164. Skyhorse Publishing, 2012.

[125] M. Casanova, E. Sokhadze, A. El-Baz, J. Baruth, G. Mathai, and L. Sears. Research at the University of Louisville Autism Center, chapter 7, pages 425-411. Skyhorse Publishing, 2012.

[126] E. Sokhadze, A. El-Baz, J. Baruth, G. Mathai, L. Sears, and M. Casanova. Effect of a low frequency repetitive transcranial magnetic stimulation (rtms) on induced gamma frequency oscillations and event-related potentials during processing of illusory figures in autism spectrum disorders. Journal of Autism and Developmental Disorders, 39(4):619-634, 2009.

[127] M. Casanova, J. Baruth, A. El-Baz, G. Sokhadze, M. Hensley, and E. Sokhadze. Evoked and Induced Gamma-Frequency Oscillations in Autism," In: Imaging the Brain in Autism, chapter 5, pages 87-106. Springer, New York, 2013.

[128] J. Baruth, M. Casanova, A. El-Baz, T. Horrell, G. Mathai, L. Sears, and E. Sokhadze. Low-frequency repetitive transcranial magnetic stimulation modulates evoked-gamma oscillations frequency in autism spectrum disorder. Journal of Neurotherapy, 3:179-194, 2010.

[129] E. Sokhadze, J. Baruth, A. El-Baz, T. Horrell, G. Sokhadze, T. Carroll, A. Tasman, L. Sears, and M. Casanova. Impaired error monitoring and correction function in autism. Journal of Neurotherapy, 14(2), 2010.

[130] E. Sokhadze, J. Baruth, A. El-Baz, R. Ramaswamy, L. Sears, and M. Casanova. Transcaranial magnetic stimulation study of gamma induction in response to illusory figures in patients with autism spectrum disorders. Journal of Neuroscience Methods, 13(4):271-272, 2009.

[131] E. Sokhadze, J. Baruth, L. Sears, G. Sokhadze, A. El-Baz, E. Williams, R. Klapheke, and M. Casanova. Event-related potentials study of attention regulation during illusory figure categorization task in adhd, autism spectrum disorders, and typical children. Journal of Neurotherapy, 16:12-31, 2012.

[132] J. Baruth, E. Williams, E. Sokhadze, A. El-Baz, L. Sears, and M. Casanova. Beneficial effects of repetitive transcranial magnetic stimulation (rtms) on 
behavioral outcome measures in autism spectrum disorder. Autism Research, pages 52-57, 2011.

[133] A. El-Baz, A. Elnakib, M. F. Casanova, G. Gimel'farb, A. E. Switala, D. Jordan, and S. Rainey. Accurate automated detection of autism related corpus callosum abnormalities. Journal of Medical Systems, 35(5):929-939, 2011.

[134] M. Mostapha, A. Alansary, A. Soliman, F. Khalifa, M. Nitzken, R. Khodeir, M. F. Casanova, and A. El-Baz. Atlas-based approach for the segmentation of infant dti mr brain images. In Proc. IEEE International Symposium on Biomedical Imaging: From Nano to Macro (ISBI'14), 2014.

[135] Y. Wang, A. El-Baz, X. Li, L. Sears, M. Casanova, A. Tasman, and E. Sokhadze. A study of relative power of specific eeg bands and their ratios during neurofeedback training in children with autism spectrum disorder. Autism Research, 2014.

[136] A. Alansary, A. Soliman, F. Khalifa, A. Elnakib, M. Mostapha, M. Nitzken, M. Casanova, and A. El-Baz. Map-based framework for segmentation of $\mathrm{mr}$ brain images based on visual appearance and prior shape. MIDAS Journal, 1:1-13, 2013.

[137] M. Casanova, M. Hensley, E. Sokhadze, A. El-Baz, Y. Wang X. Li, and L. Sears. Effects of weekly low-frequency rtms on autonomic measures in children with autism spectrum disorder. Frontiers in Human Neuroscience, 8(851), 2014.

[138] E. Sokhadze, A. El-Baz, A. Tasman, L. Sears, Y. Wang, E. Lamina, and M. Casanova. Neuromodulation integrating rtms and neurofeedback for the treatment of autism spectrum disorder. Applied Psychophysiology and Biofeedback, 39(3-4):137-257, December 2014.

[139] E. Sokhadze, A. El-Baz, L. Sears, L Opris, and M. Casanova. rtms neuromodulation improves electrocortical functional measures of information processing and behavioral responses in autism. Frontiers in Systems Neuroscience, 8, August 2014.

[140] E. Sokhadze, J. Baruth, L. Sears, G. Sokhadze, A. El-Baz, and M. Casanova. Prefrontal neuromodulation using rtms improves error monitoring and correction function in autism. Applied Psychophysiology and Biofeedback, 37(2):91102, 2012.

[141] E. M. Sokhadze, J. M. Baruth, L. Sears, G. E. Sokhadze, A. S. El-Baz, E. Williams, R. Klapheke, and M. F. Casanova. Event-related potential study of attention regulation during illusory figure categorization task in adhd autism spectrum disorder and typical children. J Neurother, 16(1):12-31, 2012.

[142] M. F. Casanova, A. Farag, A. EL-Baz, Mott Meghan, H. Hassan, R. Fahmi, and A. E. Switala. Abnormalities of the gyral window in autism: A macroscopic correlate to a putative minicolumnopathy. Journal of Special Education and Rehabilitation, 7(1-2), 2006. 
[143] R. Fahmi, A. S. El-Baz, H. AbdEl Munim, A. A. Farag, and M. F. Casanova. Classification techniques for autistic vs. typically developing brain using MRI data. In Proc. IEEE International Symposium on Biomedical Imaging: From Nano to Macro (ISBI'2007), pages 1348 -1351. IEEE, 2007.

[144] A. El-Baz, M. F. Casanova, G. Gimel'farb, M. Mott, and A. E. Switwala. A new image analysis approach for automatic classification of autistic brains. In Proceedings of IEEE International Symposium on Biomedical Imaging: From Nano to Macro, (ISBI'07), pages 352-355. IEEE, 2007.

[145] M. F. Casanova, A. S. El-Baz, and J. S. Suri. Imaging the Brain in Autism. Springer, 2014.

[146] A. A Farag, R. Fahmi, M. F. Casanova, A. E. Abdel-Hakim, H. Abd ElMunim, and A. El-Baz. Robust neuroimaging-based classification techniques of autistic vs. typically developing brain. In Deformable Models, pages 535566. Springer, 2007.

[147] M. F. Casanova, B. Dombroski, and A. E. Switala. Imaging and the corpus callosum in patients with autism. 2014.

[148] R. Fahmi, A. Elbazb, H. Hassan, A. A Farag, and M. F Casanova. Structural MRI-based discrimination between autistic and typically developing brain. pages 24-26, 2007.

[149] A. El-Baz, M. F Casanova, G. Gimel'farb, M. Mott, and A. E. Switala. Autism diagnostics by $3 \mathrm{~d}$ texture analysis of cerebral white matter gyrifications. In Proc. International Conference on Medical Image Computing and ComputerAssisted Intervention (MICCAI'2007), pages 882-890. Springer, 2007.

[150] M. F. Casanova, A. El-Baz, M. Mott, G. Mannheim, H. Hassan, R. Fahmi, J. Giedd, J. M Rumsey, A. E. Switala, and A. Farag. Reduced gyral window and corpus callosum size in autism: Possible macroscopic correlates of a minicolumnopathy. Journal of autism and developmental disorders, 39(5):751764, 2009.

[151] M. F. Casanova, A. El-Baz, E. Vanbogaert, P. Narahari, and A. Switala. A topographic study of minicolumnar core width by lamina comparison between autistic subjects and controls: Possible minicolumnar disruption due to an anatomical element in-common to multiple laminae. Brain Pathology, 20(2):451-458, 2010.

[152] M. F. Casanova, A. S. El-Baz, S. S. Kamat, B. A. Dombroski, F. Khalifa, A. Elnakib, A. Soliman, A. Allison-McNutt, and A. E. Switala. Focal cortical dysplasias in autism spectrum disorders. Acta neuropathologica communications, 1(1):67, 2013.

[153] A. Elnakib, A. El-Baz, M. Casanova, G. Gimel'farb, and A. Switala. Imagebased detection of corpus callosum variability for more accurate discrimination between dyslexic and normal brains. In Proc. IEEE International Symposium on Biomedical Imaging: From Nano to Macro (ISBI'2010), pages 109-112. IEEE, 2010. 
[154] A. Elnakib, A. Soliman, M. Nitzken, M. F. Casanova, G. Gimel'farb, and A. El-Baz. Magnetic resonance imaging findings for dyslexia: A review. Journal of Biomedical Nanotechnology, 10:2778-2805, 2014.

[155] M.J. Nitzken, M.F. Casanova, G. Gimelfarb, T. Inanc, J.M. Zurada, and A. ElBaz. Shape analysis of the human brain: A brief survey. Biomedical and Health Informatics, IEEE Journal of, 18(4):1337-1354, July 2014.

[156] A. Elnakib, A. El-Baz, M. Casanova, and A. Switala. Dyslexia diagnostics by centerline-based shape analysis of the corpus callosum. In Proc. International Conference on Pattern Recognition (ICPR'2010), pages 261-264. IEEE, 2010.

[157] M. Sen, A. Rudra, A. Chowdhury, A. Elnakib, and A. El-Baz. Cerebral White Matter Segmentation using Probabilistic Graph Cut Algorithm, chapter 2. Springer-Verlag, 2011.

[158] F. Casanova, A. El-Baz, A. Elnakib, J. Giedd, J. Rumsey, E. Williams, and A. Switala. Corpus callosum shape analysis with application to dyslexia. Translational Neuroscience, 1(2):124-130, 2010.

[159] A. Elnakib, M. Casanova, G. Gimel'farb, A. Switala, and A. El-Baz. Dyslexia diagnostics by 3-D shape analysis of the corpus callosum. IEEE Transactions on Information Technology in Biomedicine, 16(4):700-708, 2012.

[160] A. El-Baz, M. Casanova, G. Gimel'farb, M. Mott, A. Switala, E. Vanbogaert, and R. McCracken. Dyslexia diagnostics by 3D texture analysis of cerebral white matter gyrifications. In Proc. International Conference on Pattern Recognition (ICPR'2008), pages 1-4. IEEE, 2008.

[161] A. El-Baz, M. Casanova, G. Gimel'farb, M. Mott, A. Switala, E. Vanbogaert, and R. McCracken. A new cad system for early diagnosis of dyslexic brains. In Proc. International Conference on Image Processing (ICIP'2008), pages 18201823. IEEE, 2008.

[162] A. El-Baz, M. Casanova, G. Gimel'farb, M. Mott, and A. Switala. An mribased diagnostic framework for early diagnosis of dyslexia. International Journal of Computer Assisted Radiology and Surgery, 3(3-4):181-189, 2008.

[163] M. Casanova, A. El-Baz, J. Giedd, J. Rumsey, and A. Switala. Increased white matter gyral depth in dyslexia: Implications for corticocortical connectivity. Journal of Autism and Developmental Disorders, 40(1):21-29, 2010.

[164] M. Nitzken, M. F. Casanova, G. Gimel'farb, A. Elnakib, F. Khalifa, A. Switala, and A. El-Baz. 3d shape analysis of the brain cortex with application to dyslexia. In Proc. International Conference on Image Processing (ICIP'2011), pages 2657-2660. IEEE, 2011.

[165] E. L. Williams, A. El-Baz, M. Nitzken, A. E. Switala, and M. F. Casanova. Spherical harmonic analysis of cortical complexity in autism and dyslexia. Translational Neuroscience, 3(1):36-40, 2012. 
[166] A. El-Baz, G. M. Beache, G. L. Gimel'farb, K. Suzuki, and K. Okada. Lung imaging data analysis. Int. J. Biomedical Imaging, 2013.

[167] E. Hosseini Asl, J. M. Zurada, , and A. El-Baz. Lung segmentation based on nonnegative matrix factorization. pages In-press, 2014.

[168] A. El-Baz, A. A. Farag, R. Falk, and R. La Rocca. Detection, visualization and identification of lung abnormalities in chest spiral ct scan: Phase-i. In Proceedings of International conference on Biomedical Engineering, Cairo, Egypt, volume 12, 2002.

[169] A. El-Baz, A. A. Farag, R. Falk, and R. La Rocca. A unified approach for detection, visualization, and identification of lung abnormalities in chest spiral CT scans. In International Congress Series, volume 1256, pages 998-1004. Elsevier, 2003.

[170] A. El-Bazl, A. A. Farag, R. Falk, and R. La Rocca. Automatic identification of lung abnormalities in chest spiral CT scans. In Proceedings of IEEE International Conference on Acoustics, Speech, and Signal Processing, (ICASSP'03), volume 2, pages II-261. IEEE, 2003.

[171] A. A. Farag, A. El-Baz, G. Gimel'farb, and R. Falk. Detection and recognition of lung abnormalities using deformable templates. In Proceedings of the 17th International Conference on Pattern Recognition, (ICPR'04), volume 3, pages 738-741. IEEE, 2004.

[172] A. A. Farag, A. El-Baz, G. G. Gimel'farb, R. Falk, and S. G. Hushek. Automatic detection and recognition of lung abnormalities in helical CT images using deformable templates. In Proceedings of Medical Image Computing and Computer-Assisted Intervention, (MICCAI'04), pages 856-864. Springer, 2004.

[173] A. Farag, A. El-Baz, G. Gimel'farb, and R. Falk. Detection and recognition of lung nodules in spiral ct images using deformable templates and bayesian post-classification. In Proceedings of IEEE International Conference on Image Processing, (ICIP'04), volume 5, pages 2921-2924. IEEE, 2004.

[174] A. El-Baz, S. E Yuksel, S. Elshazly, and A. A. Farag. Non-rigid registration techniques for automatic follow-up of lung nodules. In Proceedings of Computer Assisted Radiology and Surgery, (CARS'05), volume 1281, pages 11151120. Elsevier, 2005.

[175] A. A. Farag, A. El-Baz, G. Gimel'farb, M. A. El-Ghar, and T. Eldiasty. Quantitative nodule detection in low dose chest ct scans: new template modeling and evaluation for cad system design. In roceedings of Medical Image Computing and Computer-Assisted Intervention, (MICCAI'05), pages 720-728. Springer, 2005.

[176] A. A. Farag, A. El-Baz, G. Gimel'farb, R. Falk, M. A. El-Ghar, T. Eldiasty, and S. Elshazly. Appearance models for robust segmentation of pulmonary nodules in $3 \mathrm{~d}$ ldct chest images. In Proceedings of Medical Image Computing and Computer-Assisted Intervention-MICCAI 2006, pages 662-670. Springer, 2006. 
[177] A. El-Baz, A. Farag, G. Gimel'farb, R. Falk, M. El-Ghar, and T. Eldiasty. A framework for automatic segmentation of lung nodules from low dose chest CT scans. In Proceedings of International Conference on Pattern Recognition, (ICPR'06), volume 3, pages 611-614. IEEE, 2006.

[178] A. M. Ali, A. S El-Baz, and A. A. Farag. A novel framework for accurate lung segmentation using graph cuts. In Proceedings of IEEE International Symposium on Biomedical Imaging: From Nano to Macro, (ISBI'07), pages 908-911. IEEE, 2007.

[179] A. El-Baz, G. Gimel'farb, R. Falk, and M. Abou El-Ghar. A novel approach for automatic follow-up of detected lung nodules. In Proceedings of IEEE International Conference on Image Processing, (ICIP'07), volume 5, pages V-501. IEEE, 2007.

[180] A. El-Baz, G. Gimel'farb, R. Falk, and M. Abou El-Ghar. A new cad system for early diagnosis of detected lung nodules. In Proceedings of IEEE International Conference on Image Processing, (ICIP'07), volume 2, pages II-461. IEEE, 2007.

[181] A. El-Baz, M. F. Casanova, G. Gimel'farb, M. Mott, and A. E. Switwala. A new image analysis approach for automatic classification of autistic brains. In Proc. IEEE International Symposium on Biomedical Imaging: From Nano to Macro (ISBI'2007), pages 352-355. IEEE, 2007.

[182] A. El-Baz, G. Gimel'farb, R. Falk, M. Abou El-Ghar, and H. Refaie. Promising results for early diagnosis of lung cancer. In Proceedings of IEEE International Symposium on Biomedical Imaging: From Nano to Macro, (ISBI'08), pages 11511154. IEEE, 2008.

[183] A. El-Baz, G. Gimel'farb, R. Falk, and M. El-Ghar. A new approach for automatic analysis of 3D low dose CT images for accurate monitoring the detected lung nodules. In Proceedings of International Conference on Pattern Recognition, (ICPR'08), pages 1-4. IEEE, 2008.

[184] A. El-Baz, G. L. Gimel'farb, R. Falk, T. Holland, and T. Shaffer. A framework for unsupervised segmentation of lung tissues from low dose computed tomography images. In Proceedings of British Machine Vision, (BMVC'08), pages 1-10, 2008.

[185] A. El-Baz, G. L. Gimel'farb, R. Falk, D. Heredis, and M. Abou El-Ghar. A novel approach for accurate estimation of the growth rate of the detected lung nodules. In Proceedings of International Workshop on Pulmonary Image Analysis, pages 33-42, 2008.

[186] A. El-Baz, G. L. Gimel'farb, R. Falk, M. Abou El-Ghar, T. Holland, and T. Shaffer. A new stochastic framework for accurate lung segmentation. In Proceedings of Medical Image Computing and Computer-Assisted Intervention, (MICCAI'08), pages 322-330, 2008. 
[187] A. M. Ali and A. A. Farag. Automatic lung segmentation of volumetric lowdose ct scans using graph cuts. In Advances in Visual Computing, pages 258267. Springer, 2008.

[188] A. El-Baz, G. Gimel'farb, R. Falk, and M. Abo El-Ghar. Automatic analysis of 3d low dose ct images for early diagnosis of lung cancer. Pattern Recognition, 42(6):1041-1051, 2009.

[189] A. El-Baz, G. Gimel'farb, R. Falk, M. Abou El-Ghar, S. Rainey, D. Heredia, and T. Shaffer. Toward early diagnosis of lung cancer. In Proceedings of Medical Image Computing and Computer-Assisted Intervention, (MICCAI'09), pages 682-689. Springer, 2009.

[190] A. El-Baz, G. Gimel'farb, R. Falk, and M. El-Ghar. Appearance analysis for diagnosing malignant lung nodules. In Proceedings of IEEE International Symposium on Biomedical Imaging: From Nano to Macro (ISBI'10), pages 193-196. IEEE, 2010.

[191] M. Kondapaneni, M. Nitzken, E. Bogaert, G. Gimel'farb, R. Falk, M. El-Ghar, and A. El-Baz. A novel shape-based diagnostic approach for early diagnosis of lung nodules. CHEST Journal, 140(4):655A-655A, 2011.

[192] A. S. El-Baz and J. S. Suri. Lung Imaging and Computer Aided Diagnosis. CRC Press, 2011.

[193] A. El-Baz, P. Sethu, G. Gimel'farb, F. Khalifa, A. Elnakib, R. Falk, and M. ElGhar. Elastic phantoms generated by microfluidics technology: Validation of an imaged-based approach for accurate measurement of the growth rate of lung nodules. Biotechnology journal, 6(2):195-203, 2011.

[194] A. El-Baz, G. Gimel'farb, R. Falk, M. El-Ghar, and J. Suri. Appearance analysis for the early assessment of detected lung nodules. In Lung Imaging and Computer Aided Diagnosis, chapter 17, pages 395-404. chapter, 2011.

[195] A. El-Baz, P. Sethu, G. Gimel'farb, F. Khalifa, A. Elnakib, R. Falk, M. El-Ghar, and J. Suri. Validation of a new imaged-based approach for the accurate estimating of the growth rate of detected lung nodules using real computed tomography images and elastic phantoms generated by state-of-theart microfluidics technology. In Lung Imaging and Computer Aided Diagnosis, chapter 18, pages 405-420. chapter, 2011.

[196] A. El-Baz, M. Nitzken, G. Gimel'farb, E. Van Bogaert, R. Falk, M. El-Ghar, and J. Suri. Three-dimensional shape analysis using spherical harmonics for early assessment of detected lung nodules. In Lung Imaging and Computer Aided Diagnosis, chapter 19, pages 421-438. chapter, 2011.

[197] A. El-Baz, M. Nitzken, F. Khalifa, A. Elnakib, G. Gimel'farb, R. Falk, and M. El-Ghar. 3d shape analysis for early diagnosis of malignant lung nodules. In Proceedings of Information Processing in Medical Imaging, (IPMI'11), pages 772-783. Springer, 2011. 
[198] B. Abdollahi, A. Soliman, A. Civelek, X-F Li, G. Gimel'farb, and A. El-Baz. A novel gaussian scale space-based joint MGRF framework for precise lung segmentation. In Proceedings of IEEE International Conference on Image Processing, (ICIP'12), pages 2029-2032. IEEE, 2012.

[199] A. El-Baz, F. Khalifa, A. Elnakib, M. Nitzken, A. Soliman, P. McClure, M. ElGhar, and G. Gimel'farb. A novel approach for global lung registration using $3 \mathrm{~d}$ markov-gibbs appearance model. In Proceeding of Medical Image Computing and Computer-Assisted Intervention, (MICCAI'12), pages 114-121. Springer, 2012.

[200] B. Abdollahi, A. Soliman, A. Civelek, X-F Li, G. Gimel'farb, and A. El-Baz. A novel 3d joint mgrf framework for precise lung segmentation. In Machine Learning in Medical Imaging, pages 86-93. Springer, 2012.

[201] A. El-Baz, G. Gimel'farb, M. Abou El-Ghar, and R. Falk. Appearance-based diagnostic system for early assessment of malignant lung nodules. In Proceedings of IEEE International Conference on Image Processing, (ICIP'12), pages 533-536. IEEE, 2012.

[202] A. El-Baz, A. Soliman, P. McClure, G. Gimel'farb, M. El-Ghar, and R. Falk. Early assessment of malignant lung nodules based on the spatial analysis of detected lung nodules. In Proceedings of IEEE International Symposium on Biomedical Imaging: From Nano to Macro, (ISBI'12), pages 1463-1466. IEEE, 2012.

[203] A. Soliman, F. Khalifa, A. Alansary, G. Gimel'farb, and A. El-Baz. Segmentation of lung region based on using parallel implementation of joint mgrf: Validation on $3 \mathrm{~d}$ realistic lung phantoms. In Proceedings of International Symposium on Biomedical Imaging: From Nano to Macro, (ISBI'13), pages 864-867. IEEE, 2013.

[204] A. Soliman, F. Khalifa, A. Alansary, G. Gimel'farb, and A. El-Baz. Performance evaluation of an automatic MGRF-based lung segmentation approach. In Proceedings of International Symposium on Computational Models for Life Sciences, (CMLS'13), volume 1559, page 323, 2013.

[205] A. El-Baz, G Gimel'farb, R. Falk, and M. El-Ghar. 3D MGRF-based appearance modeling for robust segmentation of pulmonary nodules in 3D LDCT chest images. In Lung Imaging and Computer Aided Diagnosis, chapter 3, pages 51-63. chapter, 2011.

[206] A. El-Baz, G. M. Beache, G. Gimel'farb, K. Suzuki, K. Okada, A. Elnakib, A. Soliman, and B. Abdollahi. Computer-aided diagnosis systems for lung cancer: Challenges and methodologies. International Journal of Biomedical Imaging, page 46, 2013.

[207] A. El-Baz, A. Elnakib, M. Abou El-Ghar, G. Gimel'farb, R. Falk, and A. Farag. Automatic detection of $2 \mathrm{~d}$ and $3 \mathrm{~d}$ lung nodules in chest spiral ct scans. International Journal of Biomedical Imaging, 2013, 2013. 
[208] M. Mostapha, F. Khalifa, A. Alansary, A. Soliman, J. Suri, and A. El-Baz. Computer Aided Diagnosis Systems for Acute Renal Transplant Rejection: Challenges and Methodologies, chapter 1. Springer-Verlag, 2014.

[209] Luca Saba A. El-Baz and J. Suri. Abdomen and Thoracic Imaging - An Engineering and Clinical Perspective. Springer-Verlag, 2014.

[210] F. Khalifa, A. Soliman, M. Abou El-Ghar, G. Gimel'farb, R. Ouseph, A. C. Dwyer, T. El-Diasty, , and A. El-Baz. Models and methods for analyzing dce mri: A review. Medical Physics, 2014.

[211] L. Mackelaite, R. Ouseph, A. El-Baz, and A. Gaweda. Cortical ct perfusion of the live donor kidneys as a predictor of post-transplant graft function. American Journal of Transplantation, page 329, 2012.

[212] S. E. Yuksel, A. El-Baz, A. A. Farag, M. E. Abo El-Ghar, T. A. Eldiasty, and M. A. Ghoneim. Automatic detection of renal rejection after kidney transplantation. In International Congress Series, volume 1281, pages 773-778, 2005.

[213] S. E. Yuksel, A. El-Baz, and A. A. Farag. A kidney segmentation framework for dynamic contrast enhanced magnetic resonance imaging. In Proceedings of International Symposium on Mathematical Methods in Engineering, (MME'06), pages 55-64, Ankara, Turkey, April, 2006.

[214] A. Farag, A. El-Baz, S. Yuksel, M. Abou El-Ghar, and T. Eldiasty. A framework for the detection of acute rejection with Dynamic Contrast Enhanced Magnetic Resonance Imaging. In Proceedings of IEEE International Symposium on Biomedical Imaging: From Nano to Macro, (ISBI'06), pages 418-421, Arlington, Virginia, USA, April 6-9, 2006.

[215] A. El-Baz, A. Farag, R. Fahmi, S. Yuksel, W. Miller, M. Abou El-Ghar, T. ElDiasty, and M. Ghoneim. A new cad system for the evaluation of kidney diseases using dce-mri. In Proceedings of International Conference on Medical Image Computing and Computer-Assisted Intervention, (MICCAI'08), pages 446453, Copenhagen, Denmark, October, 2006.

[216] A. El-Baz, A. Farag, R. Fahmi, S. Yuksel, M. Abo El-Ghar, and T. Eldiasty. Image analysis of renal DCE MRI for the detection of acute renal rejection. In Proceedings of IAPR International Conference on Pattern Recognition (ICPR'06), pages 822-825, Hong Kong, August 20-24, 2006.

[217] A. M. Ali, A. A. Farag, and A. El-Baz. Graph cuts framework for kidney segmentation with prior shape constraints. In Proceedings of International Conference on Medical Image Computing and Computer-Assisted Intervention, (MICCAI'07), volume 1, pages 384-392, Brisbane, Australia, October 29-November 2, 2007.

[218] A. El-Baz, G. Gimel'farb, and M. Abou El-Ghar. New motion correction models for automatic identification of renal transplant rejection. In Proceedings of International Conference on Medical Image Computing and Computer-Assisted 
Intervention, (MICCAI'07), pages 235-243, Brisbane, Australia, October 29November 2, 2007.

[219] A. El-Baz, A. A. Farag, S. E. Yuksel, M. E. A. El-Ghar, T. A. Eldiasty, and M. A. Ghoneim. Application of deformable models for the detection of acute renal rejection. In A. A. Farag and J. S. Suri, editors, Deformable Models, volume 1, chapter 10, pages 293-333. 2007.

[220] S. E. Yuksel, A. El-Baz, A. A. Farag, M. El-Ghar, T. Eldiasty, and M. A. Ghoneim. A kidney segmentation framework for dynamic contrast enhanced magnetic resonance imaging. Journal of Vibration and Control, 13(910):1505-1516, 2007.

[221] A. El-Baz, G. Gimel'farb, and M. Abo El-Ghar. Image analysis approach for identification of renal transplant rejection. In Proceedings of IAPR International Conference on Pattern Recognition, (ICPR'08), pages 1-4, Tampa, Florida, USA, December, 2008.

[222] A. El-Baz, G. Gimel'farb, and M. Abo El-Ghar. A novel image analysis approach for accurate identification of acute renal rejection. In Proceedings of IEEE International Conference on Image Processing, (ICIP'08), pages 1812-1815, San Diego, California, USA, October 12-15, 2008.

[223] F. Khalifa, A. El-Baz, G. Gimel'farb, R. Ouseph, and M. A. El-Ghar. Shapeappearance guided level-set deformable model for image segmentation. In Proceedings of IAPR International Conference on Pattern Recognition, (ICPR'10), pages 4581-4584, Istanbul, Turkey, August 23-26, 2010.

[224] F. Khalifa, A. El-Baz, G. Gimel'farb, and M. Abo El-Ghar. Non-invasive image-based approach for early detection of acute renal rejection. In Proceedings of International Conference on Medical Image Computing and ComputerAssisted Intervention, (MICCAI'10), pages 10-18, Beijing, China, September 20-24, 2010.

[225] F. Khalifa, G. Gimel'farb, M. A. El-Ghar, G. Sokhadze, S. Manning, P. McClure, R. Ouseph, and A. El-Baz. A new deformable model-based segmentation approach for accurate extraction of the kidney from abdominal CT images. In Proceedings of IEEE International Conference on Image Processing, (ICIP'12), pages 3393-3396, Brussels, Belgium, September 11-14, 2011.

[226] A. Rudra, A. Chowdhury, A. Elnakib, F. Khalifa, A. Soliman, G. M. Beache, and A. El-Baz. Kidney segmentation using graph cuts and pixel connectivity. Pattern Recognition Letters, 34(13):1470-1475, 2013.

[227] F. Khalifa, M. Abou El-Ghar, Behnaz Abdollahi, Hermann Frieboes, Tarek El-Diasty, and A. El-Baz. A comprehensive non-invasive framework for automated evaluation of acute renal transplant rejection using DCE-MRI. NMR in Biomedicine, 26(11):1460-1470, 2013. 
[228] F. Khalifa, G. M. Beache, M. Abou El-Ghar, Tarek El-Diasty, G. Gimel'farb, Maiying Kong, and A. El-Baz. Dynamic contrast-enhanced MRI-based early detection of acute renal transplant rejection. IEEE Transaction on Medical Imaging, 32(10):1910-1927, 2013.

[229] M. Mostapha, F. Khalifa, A. Alansaryand A. Solimanand G. Gimel'farb, and A. El-Baz. Dynamic mri-based computer aided diagnostic systems for early detection of kidney transplant rejection: A survey. In Proceedings of International Symposium on Computational Models for Life Science, (CMLS'13), pages 297-306, Sydney, Australia, November 27-29, 2013.

[230] M. Mostapha, F. Khalifa, A. Alansary, A. Soliman, J. Suri, and A. ElBaz. Computer-aided diagnosis systems for acute renal transplant rejection: Challenges and methodologies. In A. El-Baz and L. saba J. Suri, editors, Abdomen and Thoracic Imaging, pages 1-35. Springer, 2014.

[231] M. Nguyen, J. Tinney, F. Yuan, T. Roussel, A. El-Baz, G. Giridharan, B. Keller, and P. Sethu. Cardiac cell culture model as a left ventricle mimic for cardiac tissue generation. Analytical Chemistry, 85:8773-8779, 2013.

[232] H. Sliman, A. Elnakib, G. M. Beache, A. Soliman, F. Khalifa, G. Gimelfarb, A. Elmaghraby, and A. El-Baz. A novel 4d pde-based approach for accurate assessment of myocardium function using cine cardiac magnetic resonance images. In IEEE International Conference on Image Processing (ICIP'14), pages In-press, 2014.

[233] G. M. Beache, F. Khalifa, A. El-Baz, and G. Gimel'farb. Fully automated framework for the analysis of myocardial first-pass perfusion $\mathrm{mr}$ images. Medical Physics, 41(10), 2014.

[234] F. Khalifa, G. M. Beache, G. Gimel'farb, and A. El-Baz. A novel approach for accurate estimation of left ventricle global indexes from short-axis cine MRI. In Proceedings of IEEE International Conference on Image Processing, (ICIP'11), pages 2645-2649, Brussels, Belgium, September 11-14, 2011.

[235] F. Khalifa, G. M. Beache, M. Nitzken, G. Gimel'farb, G. A. Giridharan, and A. El-Baz. Automatic analysis of left ventricle wall thickness using short-axis cine CMR images. In Proceedings of IEEE International Symposium on Biomedical Imaging: From Nano to Macro, (ISBI'11), pages 1306-1309, Chicago, Illinois, March 30-April 2, 2011.

[236] F. Khalifa, G. M. Beache, G. Gimel'farb, G. A. Giridharan, and A. El-Baz. A new image-based framework for analyzing cine images. In A. El-Baz, U. R. Acharya, M. Mirmedhdi, and J. S. Suri, editors, Handbook of Multi Modality State-of-the-Art Medical Image Segmentation and Registration Methodologies, volume 2, chapter 3, pages 69-98. Springer, New York, 2011.

[237] M. Nitzken, G. M. Beache, A. Elnakib, F. Khalifa, G. Gimel'farb, and A. ElBaz. Accurate modeling of tagged CMR 3D image appearance characteristics to improve cardiac cycle strain estimation. In Proceedings of IEEE International 
Conference on Image Processing, (ICIP'12), pages 521-524, Lake Buena Vista, Florida, September 30-October 3, 2012.

[238] M. Nitzken, G. M. Beache, A. Elnakib, F. Khalifa, G. Gimel'farb, and A. ElBaz. Improving full-cardiac cycle strain estimation from tagged CMR by accurate modeling of 3D image appearance characteristics. In Proceedings of IEEE International Symposium on Biomedical Imaging: From Nano to Macro, (ISBI'12), pages 462-465, Barcelona, Spain, May 2-5, 2012.

[239] F. Khalifa, G. M. Beache, G. Gimel'farb, and A. El-Baz. A novel cad system for analyzing cardiac first-pass MR images. In Proceedings of IAPR International Conference on Pattern Recognition (ICPR'12), pages 77-80, Tsukuba Science City, Japan, November 11-15, 2012.

[240] F. Khalifa, G. M. Beache, A. Firjani, K. C. Welch, G. Gimel'farb, and A. El-Baz. A new nonrigid registration approach for motion correction of cardiac firstpass perfusion MRI. In Proceedings of IEEE International Conference on Image Processing, (ICIP'12), pages 1665-1668, Lake Buena Vista, Florida, September 30-October 3, 2012.

[241] F. Khalifa, G. M. Beache, A. Elnakib, H. Sliman, G. Gimel'farb, K. C. Welch, and A. El-Baz. A new nonrigid registration framework for improved visualization of transmural perfusion gradients on cardiac first-pass perfusion MRI. In Proceedings of IEEE International Symposium on Biomedical Imaging: From Nano to Macro, (ISBI'12), pages 828-831, Barcelona, Spain, May 2-5, 2012.

[242] F. Khalifa, G. M. Beache, A. Elnakib, H. Sliman, G. Gimel'farb, K. C. Welch, and A. El-Baz. A new shape-based framework for the left ventricle wall segmentation from cardiac first-pass perfusion MRI. In Proceedings of IEEE International Symposium on Biomedical Imaging: From Nano to Macro, (ISBI'13), pages 41-44, San Francisco, CA, April 7-11, 2013.

[243] H. Sliman, F. Khalifa, A. Elnakib, A. Soliman, G. M. Beache, G. Gimel'farb, A. Emam, A. Elmaghraby, and A. El-Baz. Accurate segmentation framework for the left ventricle wall from cardiac cine MRI. In Proceedings of International Symposium on Computational Models for Life Science, (CMLS'13), volume 1559, pages 287-296, Sydney, Australia, November 27-29, 2013.

[244] H. Sliman, F. Khalifa, A. Elnakib, A. Soliman, G. M. Beache, A. Elmaghraby, G. Gimel'farb, and A. El-Baz. Myocardial borders segmentation from cine MR images using bi-directional coupled parametric deformable models. Medical Physics, 40(9):1-13, 2013.

[245] H. Sliman, A. Elnakib, G. Beache, A. Elmaghraby, and A. El-Baz. Assessment of myocardial function from cine cardiac mri using a novel $4 \mathrm{~d}$ tracking approach. Journal of Computer Science and Systems Biology, 7(5):169-173, 2010. 


\section{APPENDIX I}

\section{A. LIST OF ABBREVIATIONS}

The following convention is used throughout the dissertation.

$1 \mathrm{D}$

$2 \mathrm{D}$

$3 \mathrm{D}$

$4 \mathrm{D}$

ACC

ADC

AIF

AAM

CA

CAD

CE

CE-CT

CT

CTA

DCE-CT

DCE-MRI

DG

DCT
One-Dimensional.

Two-Dimensional.

Three-Dimensional.

Four-Dimensional.

Accuracy.

Apparent Diffusion Coefficient.

Arterial Input Function.

Active Appearance Model.

Contrast Agent.

Computer-Assisted Diagnosis.

Contrast-Enhanced.

Contrast-Enhanced Computed Tomography.

Computed Tomography.

Computed Tomography Angiography.

Dynamic ContrastEnhanced Computed Tomography.

Dynamic ContrastEnhanced Magnetic Resonance Imaging.

Discrete-Gaussian.

Discrete Cosine Transform. 
DSC Dice similarity coefficients.

DTI Diffusion Tensor Image.

DWI Diffusion-Weighted Image.

ECF Extracellular Fluid Space.

EM expectation Maximization.

fMRI Functional Magnetic Resonance Imaging.

FN False Negative.

FP False Positive.

FOV Field of View.

GGMRF Generalized Gauss-Markov Random Field.

ICA Independent Component Analysis.

LCDG Linear Combinations of Discrete Gaussians.

MGRF Markov Gibbs Random Field.

MI Mutual Information.

MRI Magnetic Resonance Imaging.

MRA Magnetic Resonance Angiography.

MRS Magnetic Resonance Spectroscopy.

MRSI Magnetic Resonance Spectroscopy Imaging.

NCC Normalized Cross-Correlation.

NGF Normalized Gradient Field.

NMI Normalized Mutual Information.

PDE Partial Differential Equation.

PDMD Phase Difference Movement Detection .

PET Positron Emission Tomography.

PK Pharmacokinetic.

PPV Positive Predictive Value.

PWI Perfusion-Weighted Images.

PZ Peripheral Zone. 
ROC Receiver Operating Characteristic.

ROI Region-of-Interest.

RF Radio Frequency.

SD Standard Deviation.

SPECT Single Photon Emission Computed Tomography.

SEN Sensitivity.

SNR Signal-to-Noise Ratio.

SPE Specificity.

SVM Support Vector Machine.

TIC Time-Intensity Curve.

TN True Negative.

TZ Transition Zone.

TP True Positive.

TRUS Transrectal Ultrasound.

US Ultrasound. 


\section{APPENDIX II}

\section{A. NOMENCLATURE}

The following convention is used throughout the dissertation

- $T_{1}$ is the spin-lattice (longitudinal) relaxation time of an MRI scan.

- $T_{2}$ is the spin-spin (transverse) relaxation time of an MRI scan.

- $K^{\text {trans }}$ is the volume transfer constant of the contrast agent from the blood plasma to the interstitial space.

- $K_{e p}$ is the reverse transfer constat of the contrast agent from the interstitial space to the blood plasma.

- $(x, y)$-denotes the cartesian coordinates of points (pixels) in the image plane.

- $t$ denotes the continuous time.

- $\tau$ denotes a time step.

- $n$ denotes a discrete time instant.

- $\nabla=\left[\frac{\partial}{\partial x}, \frac{\partial}{\partial y}, \frac{\partial}{\partial z}\right]$ is the differential Operator.

- $V_{n}(x, y)$ is the deformable model speed function at each pixel location.

- $Q$ is the number of gray levels. 
- $\mathbf{Q}=\{0, \ldots, Q-1\}$ denote sets of gray levels $q$.

- $\mathbf{K}=\{1, \ldots, K\}$ denotes sets of region labels $k$.

- $\mathbf{R}$ denotes a 2-D (x,y)- arithmetic lattice.

- $\mathbf{m}$ denote binary region maps.

- $\mathrm{g}$ denote gray level images.

- $K$ is the number of image modes (number of classes).

- $P(\mathbf{g}, \mathbf{m})$ is a joint two-level probability model of a gray scale images and their region maps.

- $P(\mathbf{g} \mid \mathbf{m})$ is a conditional distribution of images, given the map.

- $P_{s}(\mathbf{m})$ is an unconditional probability distribution of maps.

- $P_{s p}(\mathbf{m})$ is shape prior probability of region maps.

- $p(q)$ is the estimated density for the mixed gray level distribution.

- $p(q \mid k)$ is the estimated marginal density for a class $k$.

- $\psi(q \mid \theta)$ is a Gaussian density with a shorthand notation $\theta \equiv\left(\mu, \sigma^{2}\right)$ for its mean, $\mu$, and variance, $\sigma^{2}$.

- $\Phi_{\theta}(q)$ is a cumulative Gaussian probability function with a shorthand notation $\theta=\left(\mu, \sigma^{2}\right)$ for its mean, $\mu$, and variance, $\sigma^{2}$.

- $C_{p}$ is the number of positive Gaussian kernel for the estimated mixed density.

- $C_{n}$ is the number of negative Gaussian kernel for the estimated mixed density.

- $w_{\mathrm{p}, \text {. }}$ is the mixed weight of positive Gaussian kernel.

- $w_{\mathrm{n}, \text {. }}$ is the mixed weight of negative Gaussian kernel. 
- $a$ is the Euclidean distance between two pixel locations.

- $\mathbf{A}=\{1, \sqrt{2}, \sqrt{3}\}$ denotes a set of Euclidean distances.

- $\mathbf{N}$ is the neighborhood system.

- $\left|\mathbf{C}_{\mathbf{N}}\right|$ is the cardinality of the neighborhood system.

- $Z$ is the normalizing factor or partition function.

- $V_{a}=\left\{V_{a, \mathrm{eq}}, V_{a, \mathrm{eq}}\right\}$ denotes the Gibbs potentials.

- $f_{a, \text { eq }}($.$) denotes the relative frequency of the equal label pairs in the equivalent$ pixel pairs in a certain neighborhood.

- $\xi$ is the shift in $x$-direction.

- $\eta$ is the shift in $y$-direction.

- $\Omega_{1: x, y}$ is the combined shape, spatial, and intensity probabilities of the object.

- $\Omega_{0: x, y}$ is the combined shape, spatial, and intensity probabilities of the background.

- $\kappa$ is the contour curvature.

- $\gamma$ denotes a scalar field.

- $E_{x}$ is the gradient vector in $x$-direction.

- $E_{y}$ is the gradient vector in $y$-direction.

- G denotes the expert, ground truth segmented region.

- $\mathrm{C}$ denotes the model segmented region.

- $A_{z}$ denotes the area under the curve.

- $\Pi$ denotes a lattice of control points. 
- $\lfloor\varepsilon\rfloor$ denotes the integer part of a real-valued number $\varepsilon$.

- $(s, t): s=x-\lfloor x\rfloor \in[0,1)$ and $t=y-\lfloor y\rfloor \in[0,1)$, is the relative position of the cartesian point $(x, y)$ with respect to the four nearest lattice points $(u, v),(u+$ $1, v),(u, v+1),(u+1, v+1)$

- $\beta_{j}(),. j=-1, \ldots, 2$, is the uniform cubic B-spline basis function.

- $C(.,$.$) is the similarity metric between the reference and target images.$

- $H($.$) is the Shannon's entropy of the image signals.$

- $H(.,$.$) is the joint entropy of the image signals.$

- $\mathcal{N}$ is the number of images in the training data.

- $\alpha$ is the shape model mixing weights.

- $\rho$ is the normalized cross correlation. 


\title{
CURRICULUM VITAE
}

\author{
Ahmad A. Firjani \\ BioImaging Laboratory \\ Paul C. Lutz Hall, Room 304 \\ University of Louisville, Louisville, KY, \\ USA \\ E-mail: aafirj01@louisville.edu \\ github.com/elmargb \\ Tel: (502) 852-4032
}

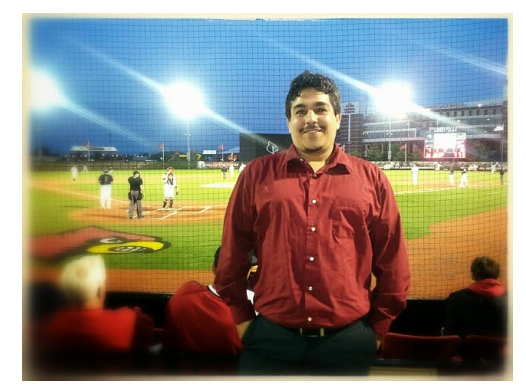

\section{Education}

2008-2014 PhD Student,Computer Engineering and Computer Science Department, University of Louisville, Louisville, KY 40292

Ph.D. Dissertation: A NON-INVASIVE IMAGE BASED SYSTEM FOR EARLY DIAGNOSIS OF PROSTATE CANCER.

2000-2002 M.Sc., in Computer and Communications Engineering, University of Putra Malaysia, Serdang, Malaysia.

M.Sc. Thesis: A Web-Based Control and Monitoring System.

1994-1999 B.S. in Electronics and Communications Engineering, Al Mergeb University, Al-Khums, Libya.

Sr. Project: DSP-based Software Radio for FM Demodulation.

\section{Teaching Experience}
2011-Present Graduate teaching assistants, Dep. of Computer Engineering and Computer Science (CECS), University of Louisville, KY, USA.
2014-Present Adjunct Professor of Computer Science, Bellarmine University, Louisville, KY, USA.
2014-Present Adjunct Faculty, Sullivan University, Louisville, KY, USA.
2002-2007 Lecturer, Department of Communications and computer Engineering,
Work Experience Al-Mergeb University, Al-Khoms, Libya.

2013- Present Software Engineer, Research MRI Center, Department of Radiology, University of Louisville Hospital, Louisville, KY, USA.

2008-2009 Software Engineer, Sleep and Neurobiology, Kosairs Children Hospital Research Institute, Division of Pediatric Sleep Medicine, Department of Pediatric, School of Medicine, University of Louisville, Louisville, KY, USA.

2001-2002 Research Assistant, Institute of Multimedia and Software , University Putra Malaysia, Serdang, Malaysia. 


\section{Professional Affiliations and Training}

- Member, Institute of Electrical and Electronics Engineers (IEEE).

Awards and Recognition

- Graduate Deans Citation Prize in recognition of excellent achievement as a candidate for advanced degree in the University of Louisville, December 2014.

- PhD Dissertation Completion Award, University of Louisville, 2014.

- Won third place in Engineering Expo competition, 2014.

- CECS Arthur M. Riehl Award, University of Louisville for excellent academic performance and creativity in research 2013.

- Citation Paper Award from the Society of UroRadiology (SUR), 2011.

- University of Louisville Travel Award 2010 and 2011.

- Scholarship for Graduate Study, 2007-2011, Ministry of Higher Education and Scientific Research, Libya.

\section{Invited Talks}

- A. Firjani, "Novel Stochastic Framework for Accurate Segmentation of Prostate in Dynamic Contrast Enhanced MRI. ," University of Louisville, CECS department, September $5,2014$.

- A. Firjani, "MRI Based Diagnostic System for Early Detection of Prostate Cancer," University of Louisville, Radiology department, April, 2014.

- A. Firjani, "Medical Image Analysis," Bellarmine university, Louisville, USA, September, 2014.

\section{Publications}

During my PhD. (Fall 2008Fall 2014), I have authored or co-authored more than 15 technical publications that have appeared in world-renown journals; book chapters; top-rank international conferences and workshops (including Medical Image Computing and Computer-Assisted Intervention (MICCAI), International Symposium on Biomedical Imaging (ISBI), and International Conference on Image Processing (ICIP)) and abstracts. 


\section{- Book Chapters}

1. A. Firjani, F. Khalifa, A. Elnakib, G.L. Gimel'farb, M. Abou El-Ghar, A. Elmagharby, and A. El-Baz, "A Novel Image-based Approach for Early Detection of Prostate Cancer using DCE-MRI," Computational Intelligence in Biomedical Imaging, K. Suzuki, Springer-Verlag, New York, 2014, ch. 3, pp. 55-82, doi: 10.1007/978-1-4614-7245-23.

\section{- Journal Articles}

1. A. Firjani, A. Elnakib, F. Khalifa, G. Gimel'farb, M. Abo El-Ghar, A. Elmaghraby, and A. El-Baz, "(2013) A diffusion-weighted imaging based diagnostic system for early detection of prostate cancer," Journal of Biomedical Science and Engineering, vol. 6, pp. 346-356.

\section{- Peer-Reviewed Conference Proceedings}

1. A. Firjani, A. Elmaghraby, and A. El-Baz, "MRI Based Diagnostic System for Early Detection of Prostate Cancer," In: Proc. of Biomedical Science and Engineering Conference (BSEC2013), Oak Ridge,Tennessee, May 21-23, 2013, pp. 1-4.

2. F. Khalifa, G. Beache, A. Firjani, K. Welch, G. Gimel'farb, and A. El-Baz, "A New Nonrigid Registration Approach For Motion Correction of Cardiac FirstPass Perfusion MRI," In: Proc. IEEE International Conference on Image Processing (ICIP'12), Orlando, Florida, September 30 -October 3, 2012, pp. 1665-1668.

3. A. Firjani, F. Khalifa, A. Elnakib, G.L. Gimel'farb, M. Abou El-Ghar, A. Elmagharby, and A.El-Baz "A Novel Image-Based Approach for Early Detection of Prostate Cancer using Diffusion Weighted MRI," In: Proc. of IEEE International Conference on Image Processing (ICIP'12), Orlando, Florida, September 30 -October 3, 2012, pp. 2849-2852.

4. A. Firjani ,F. Khalifa, A. Elnakib, G. Gimel'farb, M. Abo El-Ghar, A. Elmaghraby, and A. El-Baz, "Non-invasive image-based approach for early detection of prostate cancer," In: Proc. The Fourth International Conference on Developments in eSystems Engineering (DeSE'11), Dubai, UAE, December 6-8, 2011, pp. 172177.

5. A. Firjani, F. Khalifa, A. Elnakib, G. Gimel'farb, M. Abo El-Ghar, A. Elmaghraby, and A. El-Baz, "A new 3D automatic segmentation framework for early diagnosis of prostate cancer," In: Proc. IEEE International Conference on Image Processing (ICIP'11), Brussels, Belgium, September 11-14, pp. 2333-2336.

6. A. Firjani, A. Elnakib, F. Khalifa, G. Gimel'farb, M. Abo El-Ghar, A. Elmaghraby, and A. El-Baz, "A new 3D automatic segmentation framework for accurate extraction of prostate from diffusion imaging," In: Proc. 2011 Biomedical Science and Engineering Conference - Image Informatics and Analytics in Biomedicine (BSEC'11, Knoxville, Tennessee, USA, March 15-17, 2011, pp. 1-4.

7. A. Firjani, A. Elnakib, F. Khalifa, G. Gimel'farb, M. Abo El-Ghar, J. Suri, A. Elmaghraby, and A. El-Baz, "A new 3D automatic segmentation framework for accurate extraction of prostate from DCE-MRI," In: Proc. IEEE International Symposium on Biomedical Imaging: From Nano to Macro (ISBI'11), Chicago, Illinois, USA, 30 March - 2 April, 2011, pp. 1476-1479. 
8. A. Firjani, A. Elnakib, F. Khalifa , A. El-Baz, G. Gimel'farb, M. Abo El-Ghar, and A. Elmaghraby, "A novel 3D segmentation approach for segmenting the prostate from dynamic contrast enhanced MRI using current appearance and learned shape prior," In: Proc. IEEE International Symposium on Signal Processing and Information Technology (ISSPIT'10), Luxor, Egypt, December 15-18, 2010, pp. 137-143.

9. A. Firjani, A. Elnakib, A. El-Baz, G. Gimel'farb, M. Abo El-Ghar, and A. Elmaghraby, "Novel Stochastic Framework for Accurate Segmentation of Prostate in Dynamic Contrast Enhanced MRI," In: Proc. of International Conference on Medical Image Computing and Computer-Assisted Intervention (MICCAI'10), Beijing, China, September 20 - 24, 2010, pp. 121-130.

10. Ahmed Abdusalam, Abd Rahman R., and Liakot A., "Real Time Data Acquisition and Remote Controlling," In: Proc. of Student Conference on Research and Development, (SCOReD 2002), Selangor, Malaysia, pp. 256-259.

11. Ahmed Abdusalam, Abd Rahman R.,Nor Kamariah N., and Liakot A., "Evaluation of Power Line Communications Performance in CEBus Protocols," In: Proceedings of World Engineering congress, Sarawak, Malaysia, July 2002, pp. 2124.

\section{- Abstracts Published in Proceedings}

1. M. Abou El-Ghar, A. El-Baz, F. Khalifa, A. Elnakib, A. Firjani, and T. El-Diasty Non-invasive image-based approach for early diagnosis of prostate cancer, In: Proceedings of the European Society of Urogenital Radiology Symposium (ESUR11), Dubrovnik, Croatia, October 1316, 2011. (Citation paper award). 